University of Louisville

ThinkIR: The University of Louisville's Institutional Repository

Electronic Theses and Dissertations

$12-2014$

\title{
Self-regulation theory and self-monitoring of blood glucose behavior in type 2 diabetes mellitus.
}

Jennifer E. F. Ward

University of Louisville

Follow this and additional works at: https://ir.library.louisville.edu/etd

Part of the Analytical, Diagnostic and Therapeutic Techniques and Equipment Commons, and the Endocrinology, Diabetes, and Metabolism Commons

\section{Recommended Citation}

Ward, Jennifer E. F., "Self-regulation theory and self-monitoring of blood glucose behavior in type 2 diabetes mellitus." (2014). Electronic Theses and Dissertations. Paper 1732.

https://doi.org/10.18297/etd/1732

This Doctoral Dissertation is brought to you for free and open access by ThinkIR: The University of Louisville's Institutional Repository. It has been accepted for inclusion in Electronic Theses and Dissertations by an authorized administrator of ThinkIR: The University of Louisville's Institutional Repository. This title appears here courtesy of the author, who has retained all other copyrights. For more information, please contact thinkir@louisville.edu. 
SELF-REGULATION THEORY AND SELF-MONITORING OF BLOOD GLUCOSE

\title{
BEHAVIOR IN TYPE 2 DIABETES MELLITUS
}

\author{
By \\ Jennifer E. F. Ward \\ B.S., Centre College, 2006 \\ M.A., University of Louisville, 2012
}

\begin{abstract}
A Dissertation
Submitted to the Faculty of the

College of Arts and Sciences of the University of Louisville

in Partial Fulfillment of the Requirements

for the Degree of
\end{abstract}

Doctor of Philosophy

Department of Psychological and Brain Sciences

University of Louisville

Louisville, KY

December 2014 

SELF-REGULATION THEORY AND SELF-MONITORING OF BLOOD GLUCOSE

BEHAVIOR IN TYPE 2 DIABETES MELLITUS

By

Jennifer E. F. Ward

B.S., Centre College, 2006

M.A., University of Louisville, 2012

A Dissertation Approved on

October 1, 2014

by the following Dissertation Committee:

Barbara A. Stetson, Ph.D.

Tamara L. Newton, Ph.D.

Paul J. Rosen, Ph.D.

Paul G. Salmon, Ph.D.

Sri Prakash Mokshagundam, M.D. 


\section{DEDICATION}

This dissertation is dedicated to the members of the

\section{Health Behavior Change Research Program}

and their fearless leader,

\section{Barbara A. Stetson}




\section{ACKNOWLEDGEMENTS}

I would like to thank my husband for his ongoing support and encouragement throughout the entire dissertation process, and for understanding the long hours spent in the office and the plans I was unable to make. I would also like to thank my sister who generously offered her advice and time whenever it was requested and my parents for teaching me to value education and hard work. I would like to express my sincere gratitude to the staff at the Joslin Diabetes Center for being so welcoming to me and to the rest of the research team and to those that helped us conduct the recruitment for the study. I would also like to thank my dissertation committee; I am very grateful for their time and insightful guidance. And finally, I would like to once again thank the members of the Health Behavior Change Research Program at the University of Louisville for the countless hours of blood, sweat, and tears that they put into the study and my research mentor, Barbara A. Stetson, for her invaluable guidance and support and selfless perseverance. 


\section{ABSTRACT \\ SELF-REGULATION THEORY AND SELF-MONITORING OF BLOOD GLUCOSE BEHAVIOR IN TYPE 2 DIABETES MELLITUS \\ Jennifer E. F. Ward \\ October 1, 2014}

The present study examined self-monitoring of blood glucose (SMBG) as part of a selfregulatory process of health decision-making using the Self-Regulation Model of illness perceptions, or Common Sense Model. Participants were N=185 individuals with type 2 diabetes from a specialty diabetes clinic prescribed subcutaneous insulin or other injectable diabetes medication at least daily. Collected information included both medical chart data and self-report questionnaires completed prior receiving lab results. Self-care burden was generally high; the modal prescribed times per day of injecting insulin was 4 with modal SMBG recommendations of 3-4 times per day. Participants reported high adherence to prescribed medication regimens, varied aherence to diet recommednations, and low engagement in exercise. Specific hypotheses were developed to examine the relationship between illness coherence and illness control beliefs (IPQ), SMBG decisionmaking behavior, and outcomes including diabetes distress (PAID) and hemoglobin A1c level. These hypotheses were not supported. Supplemental analyses revealed that SMBG decision-making use was related to illness perceptions, including a positive relationship with personal control and coherence beliefs, but not treatment control, and a negative 
relationship with both outcome variables (A1c at baseline and PAID score). Both treatment and personal control beliefs were not associated with glucose control outcomes, suggesting that illness beliefs alone do not explain why some individuals are more successful at managing their diabetes than others. Coherence was found to differ by education level and SES and greatly vary in an otherwise relatively homogenous sample.

Study findings suggest that illness perceptions play an important role in the process of SMBG use for decision-making as it relates to glucose control and diabetes distress. Results also point to possible clinical targets such as illness coherence and diabetes distress. The study provides a foundation for future research related to SMBG as a decision-making strategy. 
TABLE OF CONTENTS

PAGE

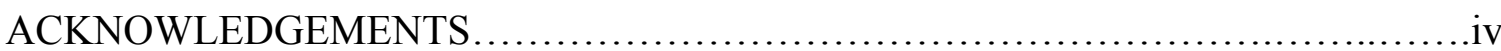

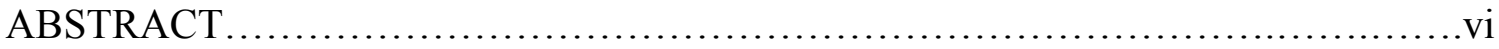

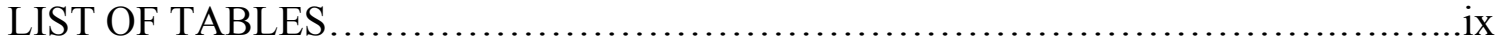

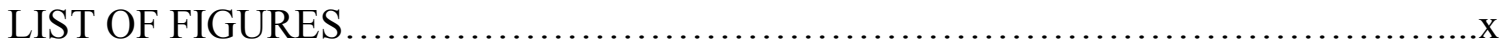

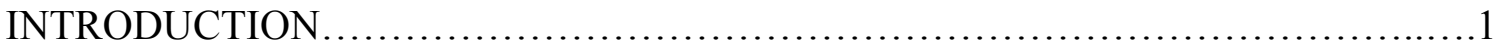

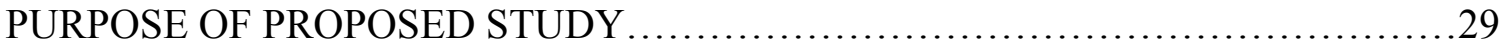

METHODS …........................................................................ 31

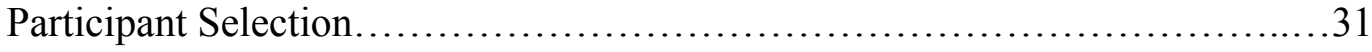

Incentive for Participation............................................... 32

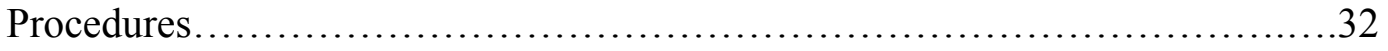

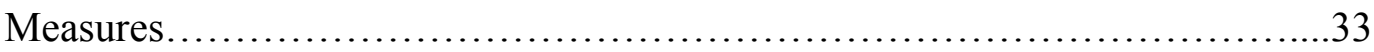

Data Analysis............................................................ 38

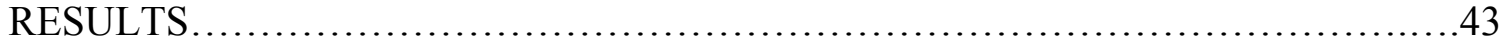

Participant Characteristics ................................................. 43

Primary Study Variables.................................................. 47

Differences in Psycho-Socio Demographic Variables Among

Primary Study Variables................................................... 51

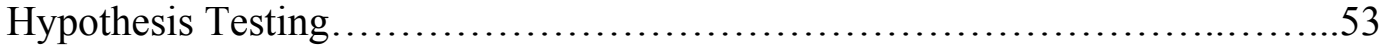

Supplemental Analyses................................................56

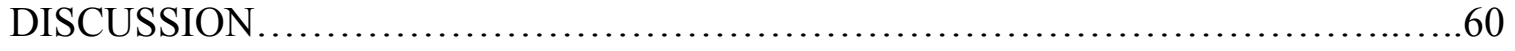

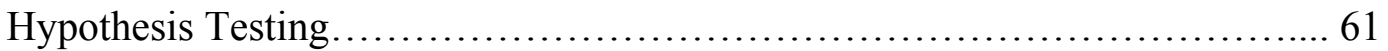


Support for the Self-Regulation Model for SMBG......................... 66

Decision-Making as a Critical Distinction in SMBG Behavior................. 73

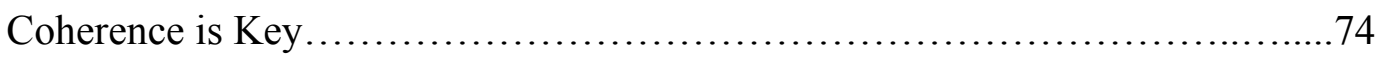

Distress is Not Related to Glucose Control...............................75

Treatment Control Beliefs and Time Since Diagnosis....................... 76

Personal Control and Treatment Control Beliefs Differ...................... 76

Strengths and Limitations.......................................... 77

Implications and Recommendations $\ldots \ldots \ldots \ldots \ldots \ldots \ldots \ldots \ldots \ldots \ldots \ldots \ldots \ldots \ldots$

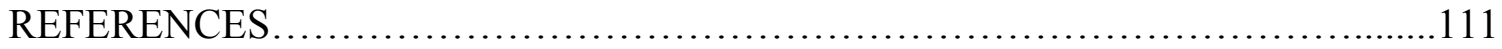

APPENDICES............................................................ 124

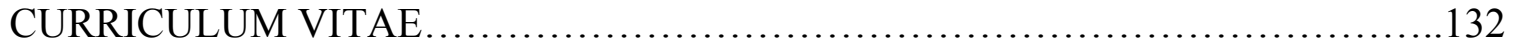




\section{LIST OF TABLES}

TABLE

PAGE

1. Statistical Procedures for Testing Hypotheses with the Joint Significance Test......84

2. List of Measures Assessing Self-Regulation Model Constructs........................85

3. Descriptive Information and Associations with Primary Study Variables for Participants Returning Questionnaires

4. Descriptive Information for Participants Not Returning Questionnaires...............89

5. Descriptive Information and Comparisons Between Participants Completing Questionnaires and Those Who Did Not....................................... 92

6. Diabetes Self-Care Behaviors (SDSCA) Descriptive Information and Associations with Primary Variables..................................................... 94

7. Primary Study Variable Descriptive Information and Associations using Correlation

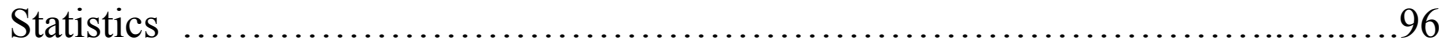

8. Regression Results for Testing of Hypothesis 1 predicting Diabetes Distress..........98

9. Regression Results for Testing of Hypothesis 2 predicting Diabetes Distress..........99

10. Regression Results for Testing of Hypothesis 3 predicting Glucose Control.........100

11. Regression Results for Testing of Hypothesis 4 predicting Glucose Control........101 


\section{LIST OF FIGURES}

FIGURE

PAGE

1. The Self-Regulation Model of Illness Behavior .............................102

2. Path diagram of the three-path mediated effect model ......................... 103

3. Standard SPSS output and hypothesis testing, example based on H1............ 104

4. Path diagram of revised simple mediation model...........................106

5. Participant flow diagram of recruitment and retention numbers................ 107

6. Path diagram of revised simple mediation model in reverse order.................108

7. Mean SMBG decision-making use by glucose control group..................109

8. Mean diabetes distress score by self-monitoring of blood glucose decision-making group............................................... 110 


\section{INTRODUCTION}

Diabetes is a group of diseases that are characterized by defects in insulin production, action or both that result in impairments in blood glucose levels. An estimated 25.8 million people in the United States have some form of Diabetes (Centers for Disease Control, 2011). Complications from Diabetes include heart disease, stroke, kidney failure, non-traumatic lower-limb amputations, and new cases of blindness (Centers for Disesase Control, 2011). Of those diagnosed with diabetes, approximately 90\%-95\% are classified as Type 2 Diabetes (T2DM; see appendix 1 for a guide to frequently-used abbreviations). T2DM is characterized by elevated circulating glucose levels resulting from either low production of insulin hormone or a resistance to the effects of insulin hormone.

Large research trials have shown that a longer period of poor glycemic control, defined as a hemoglobin A1c level (A1c) above 7\%, is associated with a higher risk for macrovascular disease (United Kingdom Prospective Diabetes Study Group, 1998), and that there is an increase in diabetes-related complications with poor glycemic control in individuals with Type 1 Diabetes Mellitus (T1DM; Diabetes Research Group, 1990). Poor control of glucose levels is associated with higher rates of neuropathy and foot problems, poor general health and well being, higher personal distress related to diabetes, higher incidence of psychological comorbidity, and higher depression scores (Fu, Qiu, \& 
Radican, 2009). Conversely, better glucose control, indicated by a lower Alc level, is associated with less microvascular complications of diabetes (American Diabetes Association, 2012). Given these results, it is imperative to learn strategies that can facilitate better, and faster control of blood glucose levels.

Diabetes is a largely self-managed disease. Responsibility for the management of diabetes care has increasingly moved away from the medical institution and toward the individual (Mc Sharry, Moss-Morris, \& Kendrick, 2011) as matters of lifestyle, such as diet and exercise, are increasingly implicated as key contributors to glucose stability. The primary self-management strategies for diabetes are outlined in the treatment recommendations from the American Diabetes Association (ADA; ADA, 2012) and the International Diabetes Federation (IDF; IDF, 2009) and include changes in diet, physical activity and the implementation of self-monitoring of blood glucose (SMBG) strategies. Type 2 diabetes is primarily treated with these lifestyle adjustments and, when necessary, oral medications and/or injectable insulin therapies.

\section{Self-Monitoring of Blood Glucose}

The remainder of this paper will focus primarily on the self-management strategy referred to as self-monitoring of blood glucose, or SMBG. Self-monitoring of blood glucose has long been considered a helpful strategy for those with diabetes. However, there has been significant debate in recent years about the appropriateness of SMBG for those with T2DM, particularly those with T2DM who are not treated with insulin therapies. Several randomized, controlled research trials have been conducted in an effort to discover whether or not this management strategy actually leads to improvements in blood glucose control (reflected by A1c) in the T2DM population (see Clar, Barnard, 
Cummins, Royle, \& Waugh, 2010 for a comprehensive systematic review). Unfortunately, the results have been split, with some studies finding significant improvements (Lalic et al., 2012) and others finding no greater improvement when compared with a control group (Clar et al., 2010). The differences have resulted in contention, more than one heated debate at scholarly conferences, and still more research aimed at identifying the errors of previous studies. There have been a number of meta-analyses, systematic literature reviews, and cost-benefit analysis attempting to find an answer to this important question (Clar et al., 2010).

\section{The Utility of Self-monitoring of Blood Glucose}

Only within the past few years has a potential consensus been proposed (Ceriello, 2012); a 2010 conceptual review identified a link between studies supporting SMBG and the use of SMBG as a decision-making tool (Kolb, Kempf, Martin, Stumvoll, \& Landgraf, 2010). A large systematic review in the same year noted that, despite significant research, there are few studies considering the use of SMBG for feedback for decision-making (Clar et al., 2010). It may be that the broad shift from a medical care focus to an increasingly self-care focus in diabetes is mirrored in the perceived utility of SMBG in the coming years. As it comes to be considered a self-care, decision-making method, it is increasingly relevant to consider the psychological processes involved in its use.

Current recommendations for SMBG use are important to consider. In current practice, there is a lack of structure in SMBG recommendations for those with T2DM who are not using insulin. The recommendations, if they are provided at all, may be as vague as 'occasionally,' 'once a day,' or 'a few times a day.' This can lead to an interesting phenomenon. In some cases, individuals my engage in perfunctory blood 
glucose testing without any planned purpose and not use the results for any feedback, doing the testing simply because their health provider instructed them to do so. Or for those engaging in SMBG with a sense of purpose, they may choose to test if, and only if, they had a concern about their blood glucose level. In this situation, it is likely that the only feedback they are receiving from SMBG are blood glucose levels that are, indeed, consistently higher or lower than the desired glucose range and therefore, contribute to a sense of "failure." Similarly, if one chooses to engage in SMBG daily, at the same time of day, they are receiving a very limited range of information. If, instead, the recommendations as to the purpose of SMBG are to test as a means of giving feedback to their healthcare provider, one may not personally attend to the results at all or might purposefully engage in SMBG when levels are in a normal range, thus receiving only 'positive' feedback and potentially leading to an inflated sense of "success." Interestingly, all of these scenarios describe an individual who is adhering to provider recommendations. It is easy to imagine how vague SMBG recommendations can lead to confusing or conflicting feedback. One reason that more structured SMBG regimens, as have been recommended very recently (Stephens et al., 2011), have proven to be successful may be because they provide more reliable feedback based on the collection of data at predetermined time points rather than self-selected time points.

Complicating the issue of SMBG recommendations is that the optimal utility of SMBG feedback is not clearly communicated. A 2010 review identified a pattern of differing perceptions of the utility of SMBG feedback between patients and their healthcare providers (Clar et al., 2010). Patients tended to expect that the primary function of SMBG results were to allow providers to make decisions about their 
treatment strategies. Conversely, healthcare providers tend to report that SMBG is primarily a decision making tool for patients to make behavioral changes (Clar et al., 2010). These perceived uses offered by both groups are valid, but rather distinct. If there is not an agreed-upon utility for SMBG across patient/provider dyads, this self-care tool may have limited effectiveness.

\section{Self-Monitoring of Blood Glucose Literature and Theory}

The recency of the shift in perceptions of SMBG utility mean that to date, little attention has been given to the psychological processes involved in SMBG use. As such, the vast majority of prior SMBG research is lacking is a conceptual foundation for the mechanisms that underlie the relationship between SMBG testing and decision-making. Some reviews of prior literature offer a conceptual framework to explain SMBG efficacy (Aalto \& Uutela,1997; McAndrew, Schneider, Burns, \& Leventhal, 2007; Wing, Epstein, Nowalk, \& Lamparski,1986), but there are few research studies built on a conceptual model. Though prior research has been helpful in providing information about overt patient- and provider-cognitions and -emotions surrounding this facet of diabetes selfmanagement, the data tends to be frequency driven and does not consider health decisionmaking. The psychological, "nonconscious" processes are an important next step for SMBG research. In health psychology literature, these processes have been consistently shown to contribute to goal selection, goal pursuit and other self-care decisions and behaviors in many illnesses (Aarts, 2007), including diabetes (Thoolen, Ridder, Bensing, Gorter, \& Rutten, 2008). The clearest understanding of the utility of SMBG in T2DM will come from research grounded in a theoretical framework, through which to explain psychological factors that are associated with its successful use are explained, and upon 
which expectations for change are based. In sum, theoretically-informed research, drawn from broader health psychology research on underlying psychological processes that explain illness behaviors as they relate to self-management choices, is a critical next step toward a greater understanding SMBG as a self-management strategy.

\section{Self-Regulation Model of Illness Behavior}

Health psychology theories for health behaviors abound. When selecting a model of illness behavior one consideration is whether it is intervention-conducive, such that it enables providers to use the information to develop interventions that can lead to improvements in the health of their patients. In a heavily self-managed illness it is also important that a conceptual model characterize the individual as able to act on their own values and goals in determining their behavior in response to a specific situation (Cameron \& Leventhal, 2003). This quality is important for applicability to an intervention because it assumes the person is an active participant in the process. Goaldirected behavior is seen as influenced not only by a cognitive process of goal selection but also by affective experience, physiological experience and social input. One such type of health behavior model is the self-regulation model.

Models of self-regulation view the self as critical in behavioral action, shaped by an ongoing process of interactions between different external and internal stimuli. As a result, these models capture a dynamic interplay between feedback, motivation and goal pursuit. The resulting feedback loops allow outcomes to serve as a reference point for success of efforts, which are motivated by a desire to maintain a physical status quo (Cameron \& Leventhal, 2003). Due to the comprehensive and dynamic nature of selfregulation theory, and the many pathways and interactions that can serve as intervention 
points, these theories are regarded as important in the research and practice in health psychology (Hagger, 2010; Hagger, Wood, Stiff, \& Chatzisarantis, 2010).

The Self-Regulation Model of illness behavior (SRM; Leventhal, 1970), one of the first models of its kind, has been specifically adapted for chronic health conditions. Like other models built on self-regulation theory, the SRM views an individual as engaging in a continuous, moment-by-moment process of decision-making that incorporates an integrative system of physical, cognitive and affective information in an effort to maintain physical balance. This adaptation posits that people develop a conceptualization, or representation, of illness, against which they continually weigh decisions about health behavior (see figure 1). Leventhal refers to this illness representation as a person's Common Sense Model (CSM; Leventhal, Leventhal, \& Contrada,1998). A person's CSM is developed across time through physical, social and cognitive input. The SRM has been studied extensively in the health population, including in individuals with diabetes (Grzywacz et al., 2011).

\section{Self-monitoring and Self-regulation}

Self-monitoring is closely related to self-regulation. The ability to self-monitor, or to monitor the results of previous action, allows the completion of the feedback loop of the SRM, which is necessary for appropriate self-regulatory action.

Specific monitoring strategies, such as SMBG, can be understood as part of the self-regulatory process as well. The use of SMBG is related to a self-regulatory process in at least two ways. One is that the self-regulatory process may lead an individual to decide to engage in SMBG as a health behavior. Another is that an individual may use the information resulting from SMBG to make decisions about future behaviors, thus 
using SMBG as a feedback aid. These two features are distinct but related; one is a resultant behavior and the other restarts the process of self-regulation. Greater selfregulation skills have been shown to be associated with a greater frequency of implementing these strategies. For example, a recent study of self-management behaviors in those with T2DM found that participants who displayed stronger cognitive abilities, such as planning and problem solving, were more likely to engage in recommended selfmanagement strategies than those whose skills are less well-developed (Primozic, Tavcar, Avbelj, Dernovsek, \& Oblak, 2012). Those who use SMBG in a self-regulatory manner, thus demonstrating a skill set, may also display more frequent engagement in SMBG behaviors.

As mentioned previously, recent analyses suggest that SMBG as a personal decision-making tool can lead to greater metabolic control than SMBG as provider feedback alone. Self-regulation processes can help explain this relationship. The feedback loop of the SRM can be characterized as making decisions about future behaviors based on output, such as a number from SMBG. In a 2010 systematic review, the five most common themes of qualitative studies related to SMBG and related barriers included a failure to act on results, a lack of education for the interpretation of data and use of data, and patient concerns that their healthcare providers were not interested in the results of their testing (Clar et al., 2010). A recent study by Lalic and colleagues (2012) examined the use of a structured SMBG protocol in a clinical setting. In addition to metabolic improvements, patients were mostly positive about all aspects of the structured format and that more than $90 \%$ noted that, through the structured format, lifestyle adjustments were easier and interactions with their provider were more positive (Lalic et al., 2012). 
Another study found greater autonomous motivation and self-efficacy ratings in those following a structured testing program compared with those in an active control group (Fisher et al., 2012). The recent trend of developing and prescribing structured testing protocols is in line with self-regulation theory. Testing at predetermined time points leaves less room for personal influence on feedback because people are not testing only when physiologically triggered.

In order to link the conceptual framework of the SRM to self-monitoring behavior in Type 2 diabetes, each portion of the SRM is discussed below, with consideration of its applicability to prior research related to SMBG.

\section{Illness Representation or Common Sense Model}

Illness Representation. It is important to remember that every person, whether a patient, a researcher, or a physician, draws from a cognitive representation when thinking about an illness. Therefore, it is a representation that is drawn from when making health decisions related to an illness, when describing an illness to others and when designing research and interventions related to the illness. Representations are not exact. Limits in human capacity for perception and cognition affect the ways in which people understand the world around them. It is reasonable to expect that very few people understand an illness in the same way. People are also constrained by contextual limitations in their understanding of an illness including the existence of, and access to, information, social influence and time. In line with these limitations, some patients may actively seek health information and become misinformed; others may rely heavily on healthcare providers for information. Importantly, the healthcare providers providing this information draw from their own illness representations. Individuals who engage in a great deal of research 
and study are not immune to limitations. There are epistemic, philosophical and political presuppositions that underlie the research that builds our understanding of medical phenomena (Gilman, 2011). Additionally, the biological indicators used to understand illness, such as A1c or blood glucose, are physiological markers of diabetes- not a direct measurement of the illness itself. Furthermore, as an illness becomes widespread, information can become even more socially relevant (Gilman, 2011) and this social influence can infiltrate the representation of even the most well intentioned empiricist.

It is important to remain cognizant of the influences that shape illness representations. Though decision-making algorithms can be developed, SMBG is not an exact science. Without frequent or continuous feedback, decisions are based on discrete time points and may not accurately represent glucose patterns.

Common Sense Model. The SRM, proposed by Leventhal (1970), includes as a major component the influence of a person's illness representation, or CSM (see figure 1). The Common Sense Model provides specificity at the 'illness representation' level. This specificity allows for easy application to previous studies and may help to explain many areas of influence previously shown to be relevant in SMBG research.

According to the SRM, there are several different categories of information people hold within their CSM of illness including identity, timeline, consequences, and cause. Specified categories result in a model that is more readily applied to health behavior research. The CSM component has been utilized in illness representation research with many chronic diseases including cancer, neurological disorders, cardiovascular disease (Heijmans \& Ridder, 1998), asthma (Tettersell, 1993) and HIV (Rungruangsiripan, Sitthimongkol, Maneesriwongul, Talley, \& Vorapongsathorn, 2011) 
and has been used to conceptualize treatment-seeking behaviors in depression (Elwy, Yeh, Worcester, \& Eisen, 2011). The CSM has been previously supported in the diabetes literature and examined for representational qualities that are unique to, or consistent with, diabetes (Grzywacz et al., 2011; Searle, Norman, Thompson, \& Vedhara, 2007). For example, a 2011 systematic review showed that previous studies have found significant associations between illness perception domains (specifically consequences, identity, timelines, coherence and emotional representations) and A1c (Mc Sharry et al., 2011). Additionally, interventions focused on targeting negative beliefs about diabetes have led to improved metabolic control (Snoek et al., 2001; as cited in Cameron \& Leventhal, 2003). These and other themes can be conceptualized within the components of the CSM. Prior research and implications will be discussed within the corresponding component below. It should be noted that discussion of a CSM related to SMBG is particularly complex given its dual relationship with the SRM. As mentioned previously, SMBG is primarily a method of feedback that begins the self-regulatory process of a separate health behavior. As such, the discussion of the common sense model of the SRM, below, will involve perceptions related to diabetes in addition to perceptions about selfmonitoring.

Identity. Identity refers to the identification with a disease or to the identity of an internal or external stimulus. Identification with diabetes as a personally experienced illness, rather than denial of the illness, would contribute to the perceived relevance of the other CSM elements. There has been some research support for a relationship between overall health perception and better adherence to SMBG; those who reported a lower or poorer perceived health status were more likely to engage in SMBG at least daily (Raffle, 
Ware, Ruhil, Hamel-Lambert, \& Denham, 2012). In other words, those who identified as being in poor health, or who had a stronger illness identity, were more likely to engage in this particular health behavior.

"Internal" and "external" stimuli refer to any number of diabetes-related stimuli. Examples of internal stimuli include physiological sensations and glycemic awareness. Internal stimuli relate to perceptions of, and persistence with, SMBG. If an individual with T2DM feels they are able to perceive physiological sensations related to their blood glucose levels, they may be less likely to view SMBG as helpful. Also, if an individual does not sense changes in their blood glucose levels, they may be less likely to be prompted to test, unless they are using a structured testing schedule.

Beliefs about internal stimuli may be supported by "sensitive soma" beliefs, or the degree to which an individual believes they are susceptible to sensing the effects (generally adverse effects) of medications (Diefenbach \& Leventhal,1996). While individuals may consider physiological sensation to be a reliable and important form of feedback in illness, people are often mistaken about the actual meaning of internal sensations or the lack thereof. Blood glucose awareness is the main target of a successful intervention related to SMBG in adults with type 1 diabetes, Blood Glucose Awareness Training (BGAT; Cox et al., 2006). This training is aimed at improving a patients' ability to regulate their blood glucose levels by way of an intervention that exposes them to the sensations they do, or do not, experience at different blood sugar levels. The intervention has been shown to improve blood glucose estimates and recognition of dangerous hypoglycemic and hyperglycemic episodes (Cox et al., 2006). 
Examples of external stimuli in identity include the recommendation to engage in SMBG and information about diabetes, as provided in educational courses or through reading. Perhaps most important is the external stimuli provided by SMBG results. After an individual engages in SMBG, they are provided a number by their monitor that indicates their current blood glucose levels. If they choose to engage, or not engage, in behaviors in response to this number, they are initiating another cycle of self-regulation. This process depends on their understanding of purpose of SMBG and their understanding of diabetes and what they can do to raise, lower, or maintain their current glucose level.

Cause. Cause refers to etiology beliefs, such as whether an illness is genetic, contracted, or injury-related. Diabetes-specific perceptions within cause have been examined in qualitative research and supported by further study. Cause perceptions tend to vary greatly between participants. In qualitative research, participants have described accurate beliefs, such as "diabetes runs in families," (p. 325) and less accurate beliefs, such as "some people get diabetes because they ate too many sweets when they were young” (Grzywacz et al., 2011, p. 325).

Little research has attended to 'cause' beliefs. Harrison and colleagues (2003) looked at cause beliefs in family members and found that perceived risk of disease development is considerably lower than actual risk in family members of a person with a positive family history of diabetes. In another study, Walter and Emery (2006) described T2DM as being generally viewed, in their sample, as an "illness of age" (p.475) and that it "felt less serious to many people who had experienced it among their relatives" (p. 475). Also, the participants who believed that T2DM is related to severe consequences tended 
to endorse higher perceived self-risk of disease development. Overall, cause beliefs tended to vary dramatically by personal experience with the outcomes and experiences of people they knew with the illness.

Timeline. Timeline refers to an understanding of the duration of a stimulus or illness. Timeline may also refer to the timescale of recovery from an illness (or lack of recovery). Perceptions of illness trajectory have been shown to relate to adherence in other diabetes literature. For example, viewing diabetes as a "non-chronic" condition diminishes the perceived importance of significant lifestyle alterations, and has been listed as a possible determinant of lack of adherence to treatments (Brown et al., 2002).

Timeline may also refer to the duration of engaging in a management strategy, such as SMBG. Beliefs related to the perceived timeline of engaging in SMBG have not been reported in prior literature. It may also be that viewing diabetes as chronic leads to the perception that SMBG is a life-long commitment. This may lead the individual to be more likely to attempt to incorporate SMBG into their lifestyle. However having a chronic illness, such as diabetes, is related to elevated depressive symptomology, reports of burden, and, at times, avoidance of management behaviors (Renn, Feliciano, \& Segal, 2011). Short-term lifestyle adaptations may seem significantly more manageable to someone who is concerned about their ability to adapt self-management strategies into their lifestyle with little impact on their quality of life. As such, more chronic timeline beliefs about SMBG may relate to lower adherence behaviors.

Consequences. Consequences refers to beliefs about the risks of the illness or treatment modality such as complications, and the possible impact on quality of life and life expectancy. The risks and long-term physiological complications of poorly managed 
diabetes are well researched (CDC, 2011). However, research on complication- and consequence-perceptions related to diabetes is lacking. Surveys on awareness of diabetes complications suggest that many individuals with T2DM underestimate their risk for heart attack, heart disease and stroke (Bairey Merz, Buse, Tuncer, \& Twillman, 2002). A recent meta-analytic review of illness perceptions and glycemic control highlighted consequences, measured by some form of the illness perceptions questionnaire (Broadbent, Petrie, Main, \& Weinman, 2006), as one of the highest predictors of glycemic control and one of the highest predictors of psychological distress compared with other components of the CSM (Mc Sharry et al., 2011).

Controllability. Since its conception, the SRM has been expanded to include two more components: controllability and coherence (Elwy et al., 2011). Controllability, also referred to as cure/control, refers to beliefs about the resources available to control the illness, including the different interventions available and their efficacy, as well as beliefs about an ability to cope with the illness or its side effects or complications (Petrie \& Weinman, 1997). The combined term controllability reflects a singular construct while cure and control refer to treatment control and personal control, respectively. Prior research supports a two-dimension conceptualization, reflecting the cure and control as separate constructs. While personal control has been found to be negatively associated with glycemic control $\left(R^{2}=-0.12\right)$, treatment control has not been consistently supported in the literature as related to glycemic control; this is possibly due to a lack of specification of treatments or not comparing those with using different treatment strategies (Mc Sharry et al., 2011). The control component of the CSM is likely more 
cohesive in relation to SMBG, particularly in those who use SMBG as a strategy for improving personal control and when treatment strategy is consistent.

In consideration of prior research findings, differences in treatment strategies, such as insulin or oral medications, should be considered when examining perceptions related to the 'control' component of the CSM. There are many treatment avenues for the physiological problems associated with T2DM; people tend differ in their beliefs about the most effective or appropriate regimen. As mentioned previously, the American Diabetes Association (ADA, 2012) and International Diabetes Federation (IDF, 2009) generally make vague care recommendations in domains in which research is not clear, such as for SMBG in T2DM. As such, it is expected that patient beliefs about the appropriateness of self-monitoring as a self-management strategy differ greatly across clinical settings. Nonetheless, most believe that some form of control is necessary (Lalic et al., 2012).

Knowledge about the skills or strategies that can facilitate glucose control has been shown to be associated with better control (Zulman, Rosland, Choi, Langa, \& Heisler, 2012). One study supported SMBG as a mediator between health literacy and better blood glucose control and also indicated that general diabetes knowledge accounted for much of the SMBG behaviors (Brega et al., 2012).

Given the personal nature of self-regulation and a tendency to over-rely on personal experience, controllability beliefs are susceptible to change if self-collected evidence appears to contradict previously held beliefs. For example, a study conducted by Lawton and colleagues (2008; as cited in Gomersall, Madill, \& Summers, 2011) found that patients who made lifestyle changes based on provider recommendations and did not 
experience improvements in their condition or face further deterioration, tended to reject the grounds on which the provider made the recommendations and were less adherent to these and future recommendations. This finding has significant implications for those who are prescribed insulin to manage their diabetes. Traditionally, insulin therapy is reserved as treatment for T2DM only when other treatment regimens, such as oral medications, fail. It may be that individuals who continue to deteriorate despite significant lifestyle changes, and are given a prescription for subcutaneous insulin, have already experienced a significant blow to their understanding of the controllability of their illness. As a result, the importance placed on provider recommendations may be lowered or they may be less likely to regularly engage in self-management behaviors.

Controllability is one component of the SRM in which the concept of selfefficacy (Bandura, 1977) may contribute to greater understanding of SMBG. Selfefficacy theory suggests that a sense of failure related to previous diabetes-management skills would contribute to low self-efficacy for other diabetes management strategies. Indeed, diabetes-specific self-efficacy has been shown to be related to better glycemic control in T2DM (Zulman et al., 2012). An individual with low diabetes self-efficacy may be particularly hesitant to engage a strategy that requires a novel skill set or that could have negative consequences if done improperly. People may be hesitant to use SMBG as feedback for adjusting behavior due to concerns about making the right decisions. However, if an individual perceives SMBG to be relatively easy to engage in, to fit in to their lives, and feel confident about what to do in the event of a high or low reading, they may be more likely to engage in the behavior. 
Additionally, glucose-monitoring format has been shown to impact maintenance behaviors. For example, the use of urine glucose monitoring has been linked to greater persistence as a strategy compared to SMBG, though quality of life was rated no differently than in the SMBG group (Lu et al., 2011). Alternative strategies should be considered in those with difficulty engaging in SMBG.

Coherence. Coherence refers to the quality of understanding, or the degree to which an individual feels they understand, an illness. Few studies in chronic health identify this component of the CSM as a primary focus, or dedicate specific questions to it; however, self-regulation theory suggests that a person's perceptions of the coherence of the illness could have great implications for treatment adherence. If an individual perceives their illness to be easily understood, they may be more likely to adapt their behaviors based on their own knowledge of the illness. One study identified that in their sample of individuals newly diagnosed with T2DM, 33\% admitted that they did not understand diabetes and $73 \%$ were unclear about which symptoms might relate to diabetes or other health concerns (Davies, Lavalle-Gonzalez, Storms, \& Gomis, 2008). If an illness is seen as confusing, the individual may feel apprehensive in instances requiring decision-making and experience affective distress or low self-efficacy.

\section{Psychosocial Factors and the Self-Regulation Model}

Several studies have found associations between affective, psychological concerns (including diabetes-specific distress), and various physiological and treatment outcomes in diabetes. A 2001 meta-analysis revealed a well-supported, significant, positive relationship between depressive symptoms and incidence of diabetes complications such as diabetic retinopathy, neuropathy, macrovascular complications and 
sexual dysfunction (De Groot, Anderson, Freedland, Clouse, \& Lustman, 2001). A 2008 meta-analysis found over forty articles supporting a positive relationship between depressive symptoms and diabetes treatment "non-adherence," including medication treatment (Gonzalez et al., 2008b). While the mechanisms underlying these relationships are unclear, it is apparent that psychological distress is an important consideration in diabetes outcomes.

Affect and coping. Unlike earlier models of health behavior (Rosenstock, 1974) the SRM considers affect as playing an important, ubiquitous role in the ongoing, moment-to-moment process of behavioral decision-making. According to the SRM, the process of health decision-making begins with a stimulus that is matched against both a person's illness representation (or CSM) and their own affective response. These two elements interact and/or result in some form of coping, which, in turn, affects an outcome (see figure 1). This process is not viewed as linear. Instead, there is an understanding of a dynamic relationship between components; feedback from the initial process may act as another stimulus, or interact with either affect or the illness representation directly.

Affect. The affective component of self-regulation is considered a process, which is methodologically difficult to capture given the potentially fleeting impact of affect. As such, affect would not be appropriately captured by a traditional self-report measure of mood, such as the Beck Depression Inventory (Beck \& Steer, 1987), that asks about mood across the last 2 weeks. Given the inherent research challenges, few studies have examined affect as it relates to diabetes behaviors. It may be, however, that anticipated affect, such as fear or guilt, proves useful in determining affective processes related to SMBG. While this is easier to measure, it assumes the individual has a level of 
psychological sophistication that enables them to reliably report associations between their mood states and behaviors.

Several studies have shown a relationship between mood and perceptions of risk for health problems. These studies show that those who are happier report less perceived risk (Salovey, Rothman, Detweiler, \& Wayne, 2000). This presents a complex issue, as risk perceptions have been shown to increase control efforts. However, self-efficacy and response efficacy, or expectations that behaviors maintain health, have both been supported as positively related to mood (Salovey et al., 2000) and potentially linked to an increase in control effort. These relationships are complex, but suggest that mood state can have a "nonconscious" effect on the CSM. This relationship is particularly relevant to self-regulation in diabetes as fluctuations in blood glucose levels are associated with idiosyncratic mood changes (Gonder-Frederick, Cox, Bobbitt, \& Pennebaker, 1989); it could be that due to mood fluctuations an individual with T2DM is more susceptible to volatile self-regulation processes, particularly if their blood glucose is not well managed. This may result in a cycle of poor control and inadequate control efforts. However, it may be that this cycle can be managed with intentional "cool" focus on the dangers of not engaging in a health behavior rather than a "warm" focus on emotion (Cameron \& Leventhal, 2003).

Coping. Coping is one of the most critical elements of the self-regulation of illness behavior. Coping occurs between the illness representation component of selfregulation and a health behavior (see figure 2). Coping is critical because it presents a potential barrier for self-regulation effort. For example, an individual can have a clear and accurate stimulus, such as a reading on the SMBG meter, and a great deal of 
knowledge about the treatment of diabetes, but fail to engage in a proactive health behavior. In order to engage in a health behavior there must be a level of adaptive coping. If this person were having difficulty coping with their illness or other concerns, the process of self-regulation may be impaired.

When examining coping as it relates to health behavior there are often a number of considerations as health behaviors can be affected by a range of concerns. In diabetes management, concerns can be behavior-specific (e.g. "I hate to stick myself"), diabetesspecific (e.g. "I feel overwhelmed by my diabetes"), or broad (e.g. "I feel hopeless"). In diabetes, difficulty coping with concerns specific to diabetes management is referred to as diabetes distress. Distress is even more common in individuals with diabetes than depression (Beverly, 2014). Some studies indicate that over half of participants report some level of diabetes-specific distress (Gonzalez et al., 2007). Distress can also be specific to individual self-management components of diabetes management. Specific concerns related to SMBG that have been previously reported are barriers to testing due to negative mood, trouble with schedules, and hating to stick oneself (Stetson, Schlundt, Rothschild, Floyd, \& Rogers, 2011). Broader reported diabetes distress concerns include worrying about complications and low blood sugar, feeling burned-out by the diabetes management regimen, and feeling depressed about having diabetes (Polonsky et al, 1995). The relationship between diabetes-specific distress and self-regulation strategies has been supported in previous research (Gonzalez et al., 2007). A recent study (Zulman et al., 2012) showed that diabetes distress was associated with poorer glycemic control in individuals with T2DM, particularly when combined with poor self-management 
strategies. This association suggests that there is a relationship between self-regulation and poor coping, although the nature of the relationship is unclear.

Coping in the SRM is most certainly impacted by mental health concerns. Depression can be difficult to separate from diabetes distress and they often coexist (Gonzalez et al., 2007). Clinically-significant depression in diabetes tends to occur at two times the rate of that of the healthy population (Gonzalez et al., 2007). There is a proposed bidirectional relationship between diabetes and depression (Renn et al., 2011). There is evidence that depression results from the difficulties of managing diabetes and also evidence that the same biological predispositions that increase the likelihood of developing diabetes also predispose one to develop depression. This bidirectional relationship would implicate both biological and behavioral processes in shaping coping ability or functioning in diabetes self-regulation. The processes may impact illness representations as well. Skinner and colleagues (2011) found that depression, as measured by a depression screener (HANDS; Zigmond et al., as cited in Skinner et al., 2011) was associated with CSM "cluster" membership. That is, depressed individuals were more likely to belong to a resisters accepting consequences cluster or a resisters cluster of CSM beliefs than more positive CSM clusters. Both clusters were related to perceptions of low personal responsibility (low personal control scores as measured on IPQ-R) and significantly higher A1c (Gonzalez, Delahanty, Safren, Meigs, \& Grant, 2008b). The findings suggest that depression may indirectly impact self-regulation by way of altering one's self opinion; an individual who is depressed may view themselves as less capable of engaging in the self-regulatory process (Cameron \& Leventhal, 2003). 
Coping in self-regulation may be modified by the practice of self-management strategies, such as SMBG. There is some evidence that receiving negative feedback from the blood-glucose testing meter, such as consistently high or low values, can contribute to a sense of failure in someone engaging in SMBG (Gomersall et al., 2011). This may be a result of the context in which an individual receives feedback from their meter. As mentioned previously, when recommendations for SMBG timing and frequency are unclear, patients are left to select random time points to test. When SMBG readings are taken at inappropriate times, results are not meaningful (St. John et al., in press, as cited in Clar et al., 2010) or may lead to overly positive or negative perceptions of control. Unfortunately, even those who have strong self-regulation skills can have difficulty with choosing the appropriate behaviors when they are receiving deceiving feedback, which may lead to difficulty coping. Diabetes distress, in particular, may be a critical factor in the self-regulation process given the high degree of diabetes distress in previous research samples and consistent associations between distress and emotional and physical diabetes wellness (Beverly, 2014).

Despite the tone of previously discussed findings, the challenges of coping with diabetes self-management practices are not insurmountable. Just as problems with coping and diabetes distress can lead to increased difficulty with managing diabetes and negative outcomes, effective coping can mitigate challenges and lead to improvements in both self-perception and physiological outcomes.

Socio-demographic variables. The SRM has been developed with a consideration for an individuals' broader socio-demographic context. Person factors, such as age and ethnicity, are considered to be highly important in shaping a person's CSM 
and self-regulation process. Diefenbach and Leventhal (1996) suggest that these factors "are more than just discrete variables, they are windows through which we can examine different processes" (p. 35). While some associations between socio-demographic variables and perceptions and behaviors related to SMBG have been revealed by prior research, this research is limited and little is known about the relationship between these factors.

A recent meta-analysis, focused on qualitative studies of general diabetes selfmanagement, highlighted gender as contributing to different health practices (Gommersall et al., 2011). Generally, men report that they are more passive with diet and other self-management practices because they view the women in their lives as traditionally taking the responsibility for their diet; conversely, women were more likely to report that they did not engage in some management strategies because they were trying to accommodate other family members.

A review of other health literature reveals that some socio-demographic variables, such as age, may critically impact illness perceptions. For example, Cameron \& Leventhal (2003) found that the aging process may lead people to attribute symptoms to their aging body rather than illness. This belief has implications for self-regulatory decisions. If a symptom is attributed to aging, they are less likely to engage in a strategy to change it.

Another consideration is the secondary impact of socio-demographic variables, such as age and BMI. For example, both older adults and those with higher BMI levels have shown impairment in their hypoglycemic awareness (Berlin, Sachon, \& Grimaldi, 2005). Lower physiological awareness, or awareness that is complicated by symptoms 
from other illnesses, can contribute to lower frequency and poorer quality of personal feedback and, therefore, poorer self-regulation.

Social and contextual factors. Social input may be seen at all levels of the SRM. Social constructs, including the healthcare system and its practices, shape the recommendations for health and wellness. The CSM portion of the self-regulation model is dependent on a system of beliefs that come from a number of sources. These sources include institutions that make medical recommendations, research conducted and supported by a body of academics, representations of illness in the media, and other social influences. An individual may or may not ascribe to the beliefs of the predominant model of health related to diabetes, the medical model, which would support all treatment recommendations of the medical, such as are outline in the ADA (ADA, 2012) or IDF guidelines (IDF, 2009).

Self-regulation skills are frequently shaped and supported by social learning, and cultural and societal values. For example, some cultures are more likely to endorse the belief that health management is the responsibility of the healthcare provider (Macaden \& Clarke, 2006 as cited in Gommersall et al., 2011), a perception which may reduce likelihood of engaging in self-regulation processes related to health. Other cultures tend to have less difficulty finding strong support networks for facilitating self-management within their culture (Keval, 2009 as cited in Gommersall et al., 2011). A single cultural context can provide both benefits and drawbacks as it relates to successful selfmanagement. Collectivist cultures, for example, tend to be characterized by stronger community and social support but can also increase the likelihood that an individual will place the needs of their family members above the their own health needs (Chun \& 
Chesla, 2004; Chesla \& Chun, 2005 as cited in Gommersall et al., 2011). Despite the evidence for a significant contribution, cultural beliefs alone do not predict selfmanagement practices. A study examining religious beliefs and diabetes selfmanagement practices found that some Christian individuals described a belief that their health and wellness was in the hands of God and adopted a passive relationship with their health, while others viewed God as a form of spiritual support and still felt actively responsible for engaging in health behaviors (Polzer \& Miles, 2007 as cited in Gommersall et al., 2011).

One of the most critical social influences in diabetes management that could presumably have a large impact on the self-regulation process is the health expert. Researchers and healthcare providers offer social input at nearly every component of the SRM. Healthcare experts impact an individual's CSM of diabetes by way of providing information that could shape illness representation components (e.g., cause or controllability beliefs). Healthcare providers can also impact the individual context by prescribing treatment regimens such as whether or not SMBG is recommended or how frequently. One study, testing a conceptual model of provider style and diabetes selfmanagement practices in a large Veteran's Affairs outpatient setting (Heisler, Bouknight, Hayward, \& Smith, 2002), found a strong relationship between a provider's communication style and patient barriers to adherence. Interestingly, the findings suggested that a patient's understanding of diabetes care tended to mediate the relationship between physician style and adherence; specifically, a more communicative style and a more interactive style were both related to higher adherence, but only when patient understanding was also high. 
There is a body of evidence that suggests diabetes practitioners are influential at the affective or coping levels of the SRM. For example, practitioners may avoid prescribing SMBG for fear of a patient's inability to emotionally and logistically cope with the addition of another health care regimen. Given the high self-management demands of diabetes, the relationship between a patient and healthcare provider may be somewhat more complex than in other illnesses. As such, social interactions between patients and providers have been regularly studied in T2DM, though none within the context of SMBG alone. Research in diabetes populations points to a complex therapeutic relationship between patients and healthcare providers. Due to the complexity of these relationships, independent constructs, such as satisfaction or communication style, are not likely to singularly capture the social contribution of providers the SRM. Indeed, a large, translational research study, the Translating Research into Action for Diabetes (TRIAD) study, found no link between diabetes-specific physiological outcomes or health behaviors (TRIAD Study Group, 2002). A more recent, theory-based longitudinal study of individuals newly diagnosed with T2DM used physiological outcomes to examine factors attributed with significant changes (Nouwen et al., 2011). Interestingly, autonomy support, an important tenet of self-determination theory, was only important in a standard diabetes self-care model, not in a model looking at changes in A1c across time. This suggests that a healthcare provider who supports the individual as the most important factor in self-care decision-making, is perceived as ideal, but does not relate to improvements in physiological outcomes. A study by Lee \& Lin (2009) supported an indirect impact of patient trust in a healthcare provider on adherence. Adherence to treatment recommendations was higher in those who rated a provider as positively 
affecting their diabetes self-efficacy expectations and outcome expectations. The evidence in these studies points to the importance of not only provider style, but also the quality of the interaction between patient and HCP. While this relationship is complex, it can also be fruitful. Patients have cited their provider as facilitating reduced psychological distress related to making changes in self-managed health behaviors (Jenkins, Hallowell, Farmer, Holman, \& Lawton, 2010).

\section{Summary}

In summary, the conceptual framework of the SRM has substantial utility in guiding understanding of SMBG in Type 2 diabetes. Illness perceptions, which may develop through social input and ones identity with diabetes and related stimuli, have particular relevance for SMBG and adaptation to life with a chronic disease. The individual components of the CSM, including causal beliefs, treatment timeline and illness consequences, may inform health-related behavioral decision-making, which is a critical part of chronic disease management. Diabetes self-management is widely recognized to require effective coping to manage the extensive demands of diabetes selfmanagement and affective responses to these and other concerns. Illness beliefs are shaped, and self-care behaviors influenced, by these considerations. Application of the SRM to SMBG, considering such illness-related perceptions as controllability and diabetes coherence, offers a conceptually driven approach to understanding SMBG as a process of self-regulation. 


\section{PURPOSE OF THE PROPOSED STUDY}

The overarching purpose of the study is to examine the use of SMBG from a selfregulatory theoretical perspective (Leventhal, 1970) and to understand its relationship to diabetes wellness. Specifically, this study aims to examine SMBG within the context of the illness perception construct of Leventhal's CSM, focusing on the domains of controllability and coherence, and their associations with two aspects of diabetes wellness, diabetes distress and metabolic control.

\section{Specific Aims}

Aim I. A primary aim of this study is to examine the use of self-monitoring of blood glucose as a mediator in the relationship between diabetes illness perceptions and diabetes wellbeing, reflected by diabetes distress.

a) To examine use of SMBG related to "controllability" as the predictor and diabetes-specific emotional coping as an outcome, and b) to examine the use of self-monitoring of blood glucose as a mediator in the relationship between diabetes illness perceptions related to "coherence" as the predictor and diabetes distress as the outcome.

Aim II. A second aim of this study is to examine the use of self-monitoring of blood glucose as a mediator in the relationship between diabetes illness perceptions and diabetes wellbeing, reflected by metabolic control (A1c). 
a) To examine use of SMBG related to "controllability" as the predictor and metabolic control (A1c) as an outcome and

b) to examine the use of self-monitoring of blood glucose as a mediator in the relationship between diabetes illness perceptions related to "coherence" as the predictor and diabetes wellbeing reflected by metabolic control (A1c).

\section{Hypotheses}

The study had a cross-sectional design with a number of hypotheses involving independent and dependent variables designed to accommodate the above aims. Specific measures and hypotheses are discussed below. 


\section{METHODS}

The research protocol was approved by the University of Louisville Institutional Review Board. The hospital-affiliated clinic recruitment site (Joslin Diabetes Center at Floyd Memorial Hospital and Health Services) deferred to the University of Louisville institutional review board for their recommendations and approval.

\section{Participant Selection}

Participants for this study were men and women with Type 2 Diabetes who were prescribed subcutaneous insulin or daily injectable diabetes medication (e.g. Byetta) and were participating in ongoing care at the Joslin Diabetes Center (JDC) at Floyd Memorial Hospital and Health Services in New Albany, Indiana. The JDC offers both educational and clinical services that are specialized for individuals with diabetes mellitus.

Participants were initially screened for a less inclusive range of age and prescription status; however, the screening criteria were expanded to reflect the typical age range of individuals with T2DM presenting for care at the JDC. Revised inclusion criteria is as follows: (1) 21 years of age or older (2) a current diagnosis of Type 2 diabetes, (3) either prescribed subcutaneous insulin therapy or daily injected diabetes medication and (4) able to read and write in English. Given the lack of prior research focused on examining this particular model as it relates to SMBG, a relatively homogeneous sample (adults, not newly diagnosed with diabetes, some experience living with an injectable medication regimen) was chosen. 


\section{Incentive for Participation}

Participants who consented to participate in the study received a reusable plastic sport water bottle (nominal value) at the time they were given the questionnaire packet. Upon questionnaire return, participants were entered into a \$250 cash lottery.

\section{Procedures}

Participant Recruitment. Participants were recruited for the study by research personnel (JW and trained graduate or undergraduate research assistants) while waiting for a scheduled blood draw appointment approximately one week in advance of a scheduled routine clinic appointment at the Joslin Diabetes Center outpatient clinic (JDC). Participants were recruited at the point of a blood draw appointment that occurred approximately one week prior to a provider visit. This point of recruitment was chosen to ensure that participant perceptions were not influenced by recent feedback from laboratory results, as this occurs during the provider visit at the JDC. All research personnel were compliant with Human Subjects Protection Program training requirements. Screening of potential subjects was completed by JDC medical staff or study investigators.

Recruitment personnel received a list of eligible participants each morning at the clinic and were alerted when the patient arrived in the private blood draw area of the clinic. The potential participant was given a brief description of the study and a flier outlining the study and asked if they were interested in meeting with personnel to discuss the study details further in a private room following their blood draw. Private offices or clinic rooms in the JDC were used for study personnel to provide further study information including a description of the study, incentives and approximate time 
commitment, and informed consent and HIPAA forms. The consent process was considered completed if the participant consented to the study and the consented participant was then provided with a packet of measures, signed copies of their consent forms, the water bottle incentive and instructions for returning the questionnaire packet in either (1) an unmarked, locked drop box in the JDC waiting area at the time of their follow-up appointment or (2) via the mail in a pre-addressed, pre-stamped return envelope. Questionnaire packets were marked with an ID number and no potentially identifying information to ensure privacy if the packet were misplaced in the mail or lost during attempted packet return. The research personnel then reminded the participant that their participation is voluntary and walked the patient to the checkout area of the clinic.

\section{Measures}

See table 2 for a summary of measures used in the study. See appendix 2 for a packet of measures used in the study.

\section{Sociodemographic Information}

Sociodemographic questionnaire. Sociodemographic information was collected from all participants via a 12-item self-report questionnaire. Information requested included age, gender, race/ethnic background, height, weight, highest level of education completed, occupation, employment status, income bracket, marital status, and current living arrangement. For those consenting to medical chart access but failing to return the questionnaire packet, basic demographic information (age, sex) was collected from their medical chart.

Medical Chart Data. Medical and health information for each consenting participant was gathered from either a computerized medical record or paper medical 
chart and stored in a de-identified SPSS data file using a subject identification number. Any identifying information linking data to the participant was kept in a separate and locked location. Collected chart data included formal endocrinology diagnosis, month/year of diabetes diagnosis, diabetes-related prescription medication prescriptions (oral diabetes-related medications, exogenous insulin), changes in insulin prescriptions before and after baseline appointment, prescribed frequency of injected medication, frequency recommended for self-monitoring of blood glucose, medical comorbidities associated with diabetes (e.g., presence of cardiovascular disease, neuropathy, peripheral neuropathy, retinopathy), previous diabetes education opportunity use or referral, prescriptions for medical and mental health comorbidities, and lab data for a prior, baseline and follow-up appointment including A1c, fasting glucose level, lipids, height, and weight.

\section{Illness Representation}

Illness Perceptions Questionnaire - Revised (IPQ-R). Illness Representation was measured using the Illness Perceptions Questionnaire - Revised (IPQ-R) formatted for diabetes (Moss-Morris et al, 2002). The IPQ-R measure consists three sections: (1) An "Identity" section with 12 items, (2) a 38-item likert-type scale section and (3) an 18-item "cause beliefs" section with an additional cause ranking item. The first section contains a list of 12 common health symptoms (e.g., "headaches," "dizziness") and 2 sets of yes/no response options, the first to indicate whether they have experienced that symptom since diagnosis, the second to indicate whether they believe that symptom is related to diabetes. The second section consists of 38 statements related to diabetes perceptions (e.g. "there is a lot which I can do to control my symptoms") and the third section contains a list of 18 
possible causes of diabetes. Response options for the two, latter sections consist of fivepoint "likert scale" response options: (0) "strongly disagree," (1) “disagree," (2) "neither agree nor disagree," (3) "agree," and (4) "strongly agree." The ranking item at the end of the third section allows short, open-responses to the prompt: "In the table below, please list in rank-order the three most important factors that you now believe caused YOUR diabetes. You may use any of the items from the box above, or you many have additional ideas of your own."

The portion of the measure used for analysis in this study consisted of 17 items, 12 items specifically related to diabetes "controllability" perceptions, divided between treatment and personal control, and 5 items related to diabetes "coherence."

Research has supported the IPQ-R as having acceptable to excellent reliability (Coherence, 0.50-0.90; Control, 0.53-0.86) (Moss-Morris et al., 2002; Skinner et al., 2011), and it has been used to measure change in illness perceptions in previous diabetes research trials (Davies et al., 2008; Skinner et al., 2011).

\section{Self-Monitoring of Blood Glucose}

Self-monitoring of blood glucose decision-making. There are few tools that measure the decision-making utility of SMBG. The measures that exist tend to focus on one aspect of decision-making, such as the use of SMBG in the process of avoiding a hyperglycemic event (HAS). As such, a one-item measure developed for a research trial, in progress, was used (D. Cox, personal communication, August $31^{\text {st }}, 2012$ ). The item is as follows: "To what extent are you using your blood sugar testing to decide what to do, like what to eat or how to be physically active, to manage your diabetes?" The response 
options for this item range from " 0 "“-"4" with "0" indicating "not at all” and "4" indicating "extremely."

Self-monitoring of blood glucose frequency. The Summary of Diabetes Self-Care Activities measure (SDSCA) was used to measure the frequency of engaging in blood glucose self-monitoring behaviors. The SDSCA is a brief, self-report measure designed for assessing diabetes self-care behaviors in both T1DM and T2DM. The measure prompts for the number of days in a given week that an individual engages in specific health behaviors, including the core self-management behaviors recommended by the ADA (ADA, 2012) and IDF (IDF, 2009). There are 11 items on the revised SDSCA that address behavioral domains including diet, exercise, blood glucose testing, foot care and smoking. The eight response options range from 0 to 7 , representing the number of days in a given week that the individual engaged in that particular behavior.

Research has suggested that the SDSCA demonstrates moderate validity and acceptable inter-item and test-retest reliability (Toobert, Hampson \& Glascow, 2000). Because items are designed to each measure an independent behavior, high reliability is not desired. The SDSCA has been previously used in studies that examine personal models of diabetes (Deakin, Cade, Williams, \& Greenwood, 2006; Hampson, Glascow, \& Toobert, 1990; Khunti et al., 2008). Only the three items related to blood glucose testing frequency were used in this study.

\section{Diabetes distress}

Problem Areas in Diabetes scale (PAID). The Problem Areas in Diabetes scale (PAID) was originally developed by Polonsky and Colleagues (1995) as a 20-item, single factor, self-report measure to assess diabetes distress in both clinical and research 
samples of individuals with diabetes. Specific items relate to feelings, perceptions and mood related to their general care regimen and living with diabetes. It is proposed that the construct measured by the sum of these responses is overall "emotional functioning" specific to diabetes (Polonsky et al., 1995), or level of diabetes distress, and has been used in medical research trials as a quality of life outcome (Davies et al., 2008). It continues to be the most frequently used, and recommended, measure of diabetes distress in the literature (Beverly, 2014).

Each item in the PAID has a response option ranging from "0" ("not a problem") to "4" ("always problem"). The total score is a summation of items that can be transformed so that scores range from 0-100 with a higher score indicating the perception of more significant and frequently encountered problems. The PAID has been found to have high internal consistency $(\alpha=.90)$ and sufficient test-retest reliability $(r=.83)$ (Welch, Weinger, Anderson, \& Polonsky, 2003). Scores on the PAID have been shown to be relatively consistent across groups, including age groups, duration of diabetes, education level, ethnicity and gender (Welch et al, 2003). PAID scores have been shown to differ between those with T1DM and T2DM diabetes but not between insulin groups (Welch, Jacobson, \& Polonsky,1997).

\section{Consideration of background variables and their associations with primary} study constructs. Previous research consistently supports differences in diabetes related behaviors and perceptions by gender, duration since diagnosis (Gomersall et al., 2011; Primozic et al., 2012), and insulin prescription status (Hampson et al., 1990). Gender differences are typically thought to interact with the process of self-regulation while age differences are thought to interact more directly with the common sense model. Gender 
and age were both assessed along with basic demographic information including socioeconomic status, educational attainment, employment and disability status and living arrangements.

T-tests or Mann-Whitney U tests or correlation tests (Pearson's or Spearman's Rho) were conducted to examine relationship of demographic and background variables with each of the independent, dependent and mediator variables. Any comparisons revealing significant differences were followed by an approach of entering that variable into regression equations in a separate block to control for its influence.

\section{Data Analysis}

Statistical analyses for descriptive information. In order to characterize the sample, a number of descriptive and univariate analyses were conducted using SPSS software version 21 (IBM Corp, 2012). Descriptive statistics included mean, standard deviation and frequency count of variables in an effort to describe the sample and check for outliers and out of range data. Binomial tests, such as Chi-square, were conducted to examine relationships between multiple discrete variables. Analyses of Variance (ANOVAs) and t-tests, where appropriate, were utilized to compare continuous variables based on divided or dichotomized sample characteristics (such as "controllability" perceptions by gender). In addition, parametric and non-parametric (Pearson and Spearman's Rho) correlation statistics were used to examine the relationship between variables (such as "controllability" perceptions and "coherence" perceptions), in order to consider applicability to past and future studies.

\section{Hypothesis testing}


Aim I. The primary aim of this study included examining the indirect relationship of illness perceptions related to "controllability" and diabetes distress through the use of self-monitoring of blood glucose, defined as two distinct properties of monitoring behavior - the use of monitoring as a decision-making strategy and the frequency of monitoring. This relationship was also examined for illness perceptions related to "coherence" in place of "controllability." See table 1 for planned statistical hypotheses corresponding to each theoretical hypothesis.

Hypothesis 1 (H1): Beliefs reflecting greater illness controllability will be indirectly associated with less diabetes distress (by way of both increased self-monitoring decision-making and higher frequency of testing).

The testing of this hypothesis (H1) was planned to be conducted in accordance with the joint significance test of mediation outlined by Taylor and colleagues (2008). The joint significance test has been shown to afford a high statistical power while maintaining a low type 1 error rate and was recommended as superior to other twomediator models. Testing H1 will consist of the use of three, separate linear regression tests to examine three separate pathways, followed by a series of critical value comparisons. The first pathway involves testing the regression of SMBG decisionmaking and controllability scores with the SMBG decision-making item as the dependent variable and scores on the personal or treatment controllability subscales of the IPQ-R (12-items) as the predictor variable. The second pathway involves regressing the frequency of SMBG (measured by the SDSCA) as the dependent variable and SMBG decision-making and controllability beliefs (IPQ-R) as the predictor variables. The final path involves a regression with emotional coping (PAID) as the dependent variable and 
controllability beliefs, SMBG frequency and SMBG decision-making as the predictor variables. Then, critical values for each regressive relationship were planned to be calculated (dividing a beta weight by its own standard error) and compared with critical values for a two-tailed t-statistic $\left(\mathrm{t}_{.975(\mathrm{n}-2)}\right)$ given a specific degree of freedom. The degrees of freedom decrease by one degree with each subsequent comparison). The null hypothesis is rejected if each calculated value $\left(b_{x} / s_{b x}\right)$ is greater than the critical $t$-statistic value. See table 1 for a summary of the statistical equations and pathways for the joint significance test. See figures 2 for a figural representation of the joint significance model with the variables from the proposed study mapped onto it. See figure 3 for the original, proposed SPSS analysis plan and data planned for final hypothesis testing.

Hypothesis 2 (H2): Beliefs reflecting greater illness coherence will be indirectly associated with less diabetes distress (by way of both increased self-monitoring decisionmaking and higher frequency of testing).

The testing of this hypothesis (H2) was planned to be conducted in accordance with the joint significance test of mediation outlined by Taylor and colleagues (2008), as described above. See table 1.

Aim II. A secondary aim of this study includes examining the indirect relationship of illness perceptions related to "controllability" on A1c through the use of self-monitoring of blood glucose, defined as two distinct properties of monitoring behavior - the use of monitoring as a decision-making strategy and the frequency of monitoring. The relationship was planned to be tested with the "coherence" subscale of the IPQ-R in place of "controllability." 
Hypothesis 3 (H3): Beliefs reflecting greater illness controllability will be indirectly associated with lower hemoglobin Alc (by way of both higher decision-making and self-monitoring frequency).

Hypothesis 4 (H4): Beliefs reflecting greater illness coherence will be indirectly associated with lower hemoglobin Alc (by way of both higher decision-making and selfmonitoring frequency).

These hypotheses ( $\mathrm{H} 3$ and $\mathrm{H} 4)$ were also planned to be tested in accordance with the joint significance test of mediation, described above. See table 1.

Sample size calculation. Taylor and colleagues (2008) conducted simulations to estimate the Type 1 error rate and power afforded by the joint significance test for a series of predetermined $(\mathrm{N}=50,100,200,500$ and 1,000) sample sizes and effect sizes (small, medium and large). As the joint significance test is a path mediation model, effect size estimates for each tested path can be multiplied and compared with simulated sample sizes to yield an estimate of power and Type 1 error rate. Based on prior related research it was estimated that the proposed study would yield a small to medium effect size (Cohen, 1992). A systematic literature review reported study characteristics for five studies comparing SMBG alone (without instructions) to SMBG with an educational feedback intervention and increased testing frequency (Clar et al., 2010). Effect sizes were calculated for the reported studies; effect sizes ranged from $\mathrm{d}=0.19$ to $\mathrm{d}=0.50 \mathrm{using}$ G*Power (Faul, Erdfelder, Buchner, \& Lang, 2009). The simulations conducted by Taylor and colleagues (2008) support the joint significance test as yielding a Type 1 error rate of less than .0060 and a power of .9120 for a sample size of 100 participants for an 
expected medium effect size $(\mathrm{d}=0.36)$ and Type 1 error rate of .0020 and a power of 0.772 for a small effect size $(\mathrm{d}=0.14)$ (MacKinnon et al., 2002).

Based on data from previous studies of persons living with T2DM collected at the JDC and standard estimates, it was anticipated that $20 \%$ of the collected data would be missing or unusable. In addition, recruitment and retention data from a previous study at this site found that $27 \%$ of individuals who consented to participate did not actually return the completed data packet. However, this study did not include a monetary incentive. Given this available data, prior to the study the recruitment target was set at 140 participants $(\mathrm{N}=100$ after approximately $20 \%$ missing /unusable data and $20 \%$ noncompleted forms). 


\section{RESULTS}

This section begins with a description of study participants and response rates. Following this will be a description of data analysis preparation and a summary of descriptive analyses and data concerns. The results of the original statistical plan for the study's four hypotheses will be discussed followed by a summary of an adjusted plan based on unforeseen data concerns, and results of the adjusted plan, by hypothesis. Finally, a number of additional, supplementary analyses examining other relationships between primary study variables will be described.

\section{Participant Characteristics}

The next few sections present participant characteristics $(\mathrm{N}=185)$ including a review of data completion and participant demographics and responses separated into domains for clarity.

Recruitment and return rates. Participant screening and recruitment numbers are presented in Figure 5. Of those patients at the JDC who were screened for the study, the majority were ineligible due to not having a type 2 diabetes diagnosis with many others not eligible due to not being prescribed insulin (likely treated with oral medications alone or managed with diet and exercise). Of those deemed eligible by chart review, the majority were not approached by research personnel; this was most often related to not having an available research assistant during the time the potential participant presented for the blood draw. 
Of those eligible patients approached by research personnel, the majority agreed to hear more about the study and consented to participate. Of those who declined to hear more about the study or to participate, the majority stated that they were 'in a hurry,' which includes responses such as "I am on my way to work and will be late," "I am fasting and hungry and can't wait to eat," or "I don't have time." Other reasons cited for not being interested in the study included discomfort with medical chart information used in the study or concerns about the time needed to complete the packet.

Of those consenting to complete the study, close to one quarter did not return their baseline questionnaire packet (27.02\%). Though limited demographic data is available for those who did not return a baseline questionnaire packet, medical chart data was obtained to allow for group comparison. Participants were not found to differ by age, body mass index, blood glucose level or Alc at prior or baseline appointments using ttest statistics (see table 5). Participants were not found to differ by sex/gender using chisquare analysis $\left(\mathrm{X}^{2}(1)=.121, \mathrm{p}=.728\right)$.

A small number of consented participants chose to revoke their authorization for collection of data (4.3\%) and another small number were found to be ineligible during medical chart review either due to no clearly documented insulin prescription or a formal diagnosis of T1DM (2.2\%).

Data completion. Of those returning baseline packets, the majority of items were completed in full. The most commonly skipped items from primary variables were related to treatment control beliefs (IPQ, $n=7$ ), SMBG decision-making $(n=6)$, illness coherence (IPQ; $\mathrm{n}=4$ ) and diabetes coping (PAID; $\mathrm{n}=4$ ). For the Problem Area in Diabetes scale (PAID), means replacement was used for up to three items; means 
replacement was used for a very small number of participants $(n=6)$. Additionally, the Illness Perceptions Questionnaire subscale related to control beliefs was divided into personal control beliefs and treatment control beliefs; the treatment control subscale had more missing data. Otherwise, cases were excluded listwise, meaning the participant's data was excluded for an entire case on an analysis-by-analysis basis. Using this method, the minimum number providing responses for planned statistical tests of the main hypotheses was $\mathrm{N}=105$, therefore, sufficient power was achieved in accordance with simulations conducted by Taylor and colleagues (2008) for the joint significance test for a predicted Type 1 error rate of less than .0060 and a power of .9120 for an expected medium effect size $(\mathrm{d}=0.36)$ and predicted Type 1 error rate of .0020 and a power of 0.772 for a small effect size $(d=0.14)$ (MacKinnon et al., 2002). For analyses involving an additional variable, treatment control (IPQ), the power may be reduced due to missing item responses $(\mathrm{N}=96)$.

Characteristics of participants who returned questionnaires. A summary of demographic and descriptive information for the study participants can be found in table 3. Participant demographics presented here are for those returning baseline packets and were confirmed as eligible to complete the study $(\mathrm{N}=124)$ as those who did not return their baseline packet have less demographic data available and are not included in the primary analyses. An analysis of differences between consented participants who returned the questionnaire packet and those who did not is discussed at the end of this section.

Socio-demographic variables. Participants were predominantly White Americans $(n=115,93.5 \%)$, which is consistent with recent area census data which indicates that 
Floyd County residents are comprised of 90.4\% White American, 5.2\% Black or African American, 0.2\% Native American, 0.9\% Asian, 0.2\% Pacific Islander, 1.2\% from other races, and $2.1 \%$ from two or more races. $2.6 \%$ of the population were Hispanic or Latino of any race (US Census, 2010). Participant gender was nearly evenly split in this sample. Ages ranged from 34 to 86 years-of-age. The majority of participants endorsed that they are currently married with at least high school grade level education or equivalent. There was a range of income levels reported though most were either disabled or retired. Habitation classification varied with most living with a spouse or partner.

Diabetes-related characteristics. The following characteristics are reported in table 3 .

Diabetes illness characteristics. There was a broad range of duration of diagnosis (from 2 to 40 years) and onset of insulin prescription (from 1 to 27 years). There is significant missing data for these variables as the onsets of the diabetes diagnosis was not always noted in the medical chart. The modal result for insulin prescription was four insulin injections daily. The modal result for times per day recommended to SMBG was 3.5 times daily (recommended as 3-4 times per day), though SMBG frequency recommendations were not always noted in the medical chart. Most participants were also prescribed oral diabetes medications. Most participants had many health comorbidities including hypertension $(n=104,7.3 \%$ missing data), heart disease $(n=24$, $43.5 \%$ missing data), hyperlipidemia ( $n=113,6.5 \%$ missing data), and peripheral neuropathy $(\mathrm{n}=56,22.6 \%$ missing data). The missing data for these variables is due to a change in medical chart data collection practices partially through the data collection process. 
Diabetes health practices. Participants indicated a range of adherence to health recommendations for diabetes self-care, as measured by the SDSCA (see table 6). Very few participants endorsed smoking even one puff of a cigarette in the last week. General adherence with oral diabetes medications was higher than adherence to guidelines for frequency of insulin injections, as measured by days in the last week the medications were taken as recommended. There was a range in responses related to how frequently participants are engaging in recommended diet plans, though several reported following recommended diet plans less than half of the previous week. Most participants reported that they engaged in a level of physical activity below the amount recommended by the American Diabetes Association standards of medical care in diabetes (ADA, 2012), with the majority engaging in physical activity (at least 30 minutes) only two days per week or less with no planned physical activity sessions.

Most participants had a history of attending Diabetes Self Management Education and many also had a history of Medical Nutrition Therapy (see table 3).

Mental health chart data. A small percentage of participants had a diagnosis of a mental health condition in their medical chart including a depressive $(\mathrm{n}=7)$, anxiety $(\mathrm{n}=4$,$) or bipolar (\mathrm{n}=1)$ disorder. A larger percentage were prescribed an antidepressant $(\mathrm{n}=36)$ anxiolytic $(\mathrm{n}=9)$ or mood stabilizer $(\mathrm{n}=7)$.

\section{Primary Study Variables}

Primary study variables were examined for their ranges, mean, medians, modes, standard deviations and response distributions and compared with other samples found in the diabetes literature (see table 7). Variables were also examined for missing data, distribution normality, and outliers. 


\section{Illness perceptions}

Personal control beliefs. Responses on IPQ items in the Personal Control Beliefs subscale were completed by participants with missing values for four cases. Data were not imputed due to the small number of items in this scale (6) and data was excluded from subsequent analyses. Responses yielded a range of scores from 17 to 30 from a possible range of $0-30$. This response range from this sample indicates that all participants reported that they consider their personal actions as having at least some impact on their diabetes outcomes, such as illness complications. The distribution of responses was moderately negatively skewed with high mean scores and a modal score of 30, indicating beliefs that personal behaviors are very important for diabetes symptoms or outcomes (see table 7). As such the variable was transformed using a square root equation for use in regression analyses in accordance with recommendations from Howell (2010) with a resulting reduction in the negative skew. Non-parametric statistics were used for all other analyses (Mann-Whitney U-test, Kruskal-Wallis H-test, or Spearman's Rho correlation).

Treatment control beliefs. Responses on the IPQ items that related to treatment control beliefs were completed by participants with missing data for 8 cases. Missing data was not imputed due to small number of items in the scale (5) and incomplete data was excluded from subsequent analyses. Responses resulted in a relatively normal distribution of scores. Scores ranged from 10 to 25 , from a possible scores range of $0-25$, indicating that all participants reported at least some degree of belief that control of their diabetes is possible. 
Diabetes coherence beliefs. Responses on the IPQ items from the illness coherence subscale resulted in a mildly negatively skewed distribution. Five participants failed to respond to items within this subscale; data was not imputed due to a small number of items in the scale (5) and the missing cases were excluded for subsequent analyses. Participant responses yielded scores ranging from 5 to 25 (subscale possible scores range from $0-25$ ), reflecting a broad range of reported personal understanding of diabetes, though the modal score was 25 .

\section{Self-monitoring of blood glucose behaviors}

Self-monitoring of blood glucose frequency. Self-monitoring of blood glucose frequency was measured by an SDSCA item that asked about days of use of SMBG in the prior week. This variable was found to be significantly negatively skewed with most participants reporting daily use over the previous week (61.0\%). Responses ranged from 0 days to 7 days. See table 7 for response results. Transformations of data were not successful in reducing skew. As such, this variable is inappropriate for use in regression analyses as a continuous variable. For regression analyses this variable will be collapsed into those testing at least 5 days per week $(\mathrm{n}=95)$ and all others $(\mathrm{n}=28)$. Only those in the first group (testing at least 5 days per week) will be considered in regression analyses for hypothesis testing.

Self-monitoring of blood glucose adherence. Self-monitoring of blood glucose adherence was measured by an SDSCA item that asked about days of use of SMBG 'the number of times recommended' in the prior week. Most reported SMBG at least daily in the past week though several reported that they did not test the number of times recommended by their provider. Due to the wording of the question and noted range of 
recommending practices noted in medical charts, it cannot be assumed that these individuals are testing less often than recommended. See table 7 for response results.

Self-monitoring of blood glucose use as a decision-making tool. Self-monitoring of blood glucose decision-making is measured by a single, self-report likert-type item reflecting extent to which the participant feels they are using their SMBG result to make decisions about their health behaviors and diabetes self-care. Responses ranged from 0 (not at all) to 4 (extremely) with that modal response at 3 (a great deal; $n=43$ ). Seven participants $(n=7)$ who returned a packet failed to respond to the item. Mean values were similar, falling between 2 (a moderate amount) and 3 (a great deal). A normality statistic found the variable to be significantly negatively skewed. However, as non-parametric tests will be used, the data was not transformed for analyses. Ordinal regression techniques (Polytomous Universal Model; PLUM) with SMBG decision-making as a dependent variable and each primary variable as a separate independent variable were used to test for violation of assumptions. No violations were found and variable was used in regression analyses without transformation or recoding. No outliers were found and all cases were retained for analyses (see table 7).

Diabetes distress. The Problem Areas in Diabetes scale (PAID) was used as a measure of diabetes distress. Four participants left a significant number of items blank and were removed from subsequent analyses and 6 participants had means replacement for up to three items ( 4 needed means replacement for 1 item, 1 needed means replacement for 2 items, and 1 needed means replacement for all 3 items). The PAID scores from this sample of participants were normally distributed with no outliers. Mean PAID scores $(\mathrm{M}=25.85, \mathrm{sd}=14.34)$ were similar to an original normative sample of 
individuals with Type 2 diabetes on insulin therapy $(M=26.4, \mathrm{sd}=20.8)$ but had a narrower standard deviation (Welch et al.,1997). All valid cases were retained for analyses.

Physiological blood glucose control. Blood glucose control was measured by A1c blood serum level (\%) drawn at the point of recruitment. Participants were not aware of their A1c at the time of packet completion. Participants were generally poorly controlled, which is consistent with individuals with Type 2 diabetes who require insulin therapies. See table 3 for descriptive information.

Examination of individual values revealed a range from $5.0 \%$ (good control) to $15.9 \%$ (very poor control). Tests of normality revealed a significant positive skew. Given that the maximum value $(15.9 \%)$ is significantly higher than the immediately preceding value of $12.5 \%$, the maximum value was removed as an outlier, which led to a more normal frequency distribution.

\section{Differences in Psycho-Socio-Demographic Variables Among Primary Study} Variables

Differences in primary study variables were examined using Pearson and Spearman's Rho correlations, T-test, or Mann-Whitney U (see tables 3 and 7). Age was found to be significantly negatively correlated with PAID scores and no other primary variables. Prescribed insulin frequency was found to be significantly positively correlated with SMBG use as a decision-making strategy and no other variables. Time since insulin prescription was negatively correlated with treatment control beliefs. Months since diabetes onset and frequency recommendations for SMBG were not found to be associated with primary variables. 
Other demographic variables were collapsed into two groups for comparison (see table 3). Differences were found between marital status groups (currently married vs. not currently married) for A1c with those married having better control. Differences were found between education groups with those having at least some college education reporting higher coherence and greater personal control beliefs than those with a high school diploma or less education. Differences were found between low income and mid to high-income groups (low income defined as currently married and under 30,000 annual household income or not married and under 20,000 annual household income) with those reporting lower annual household income also reporting lower mean coherence. No differences were found between gender groups, a dichotomized work group full-time vs. other, or those on antidepressants vs. those who are not. Ethnicity was not examined due to the largely homogeneous nature of the sample ethnicity.

Self-reported health practices (SDSCA) were examined for associations with primary study variables. Coherence and personal control beliefs were both positively associated with reports of following an eating plan and coherence beliefs was also positively associated with taking recommended number of insulin injections and spacing carbohydrate consumption across the day, see table 6 . Treatment control beliefs were positively associated with taking a recommended number of diabetes pills and eating fruits and vegetables. Use of SMBG decision-making was positively associated with frequency of taking injections as recommended and frequency of following a number of meal plans including following a healthy eating plan, eating fruits and vegetables, and spacing carbohydrates and negatively associated with frequency of eating food high in fat. Diabetes distress (PAID) was found to be negatively associated with diet behaviors 
including following an eating plan and spacing carbohydrates. Actual glucose control, as measured by A1c lab at baseline, was negatively associated with following a healthy eating plan and spacing carbohydrate and positively associated with a diet high in fats.

\section{Hypothesis Testing}

The initial statistical plan (see table 1 for the original data analytic plan divided by statistical hypothesis) was revised due to significant non-normal distribution of one of the study's primary variables, SMBG frequency. As a predicted joint mediator, SMBG frequency was present in the statistical plan for the testing of all hypotheses. Simple indirect effects tests, as described in Baron \& Kenny's model of mediation (1986) were conducted considering the relationships between the remaining three variables with the same predictor, mediator, and outcome variables as outlined in the original statistical plan with the exclusion of SMBG frequency (See figure 4 for a figural representation of the revised, proposed indirect effect pathways). Baron \& Kenny's model involves the testing of four separate regression relationships. The initial proposed relationship must be supported before subsequent relationships are tested. In order to account for SMBG frequency as part of the hypothesized relationship, the sample used in the analyses was selected based on responses from the SMBG frequency variable with only those who reported that they SMBG at least 5 out of 7 days $(n=95)$ being considered for each regression.

\section{Hypothesis 1}

Hypothesis 1 (H1): Beliefs reflecting greater illness controllability will be indirectly associated with more successful emotional coping (by way of both increased self-monitoring decision-making and higher frequency of testing). 
Hypothesis 1 was tested using two separate indirect effect pathways (Baron \& Kenny, 1986) with PAID scores as the outcome variable (Y), SMBG decision-making as the predicated statistical mediator (M), and treatment control beliefs (IPQ) as one predictor variable $(\mathrm{X})$ and personal control beliefs (IPQ) as a separate predictor variable $(\mathrm{X})$.

The first step in the first predicted pathway, testing whether personal control beliefs (IPQ) significantly account for variance in PAID scores, controlling for habitation status and education level, was not supported. No further relationships were tested in this indirect effects model. See table 8 for a summary of regression results.

The first step in the second predicted pathway, testing whether treatment control beliefs (IPQ) significantly predict PAID scores was not supported. No further relationships were tested in this indirect effects model. See table 8 for a summary of regression results.

\section{Hypothesis 2}

Hypothesis 2 (H2): Beliefs reflecting greater illness coherence will be indirectly associated with more successful emotional coping (by way of both increased selfmonitoring decision-making and higher frequency of testing).

Hypothesis 2 was tested using an indirect effects model (Baron \& Kenny, 1986) with PAID scores as the outcome variable (Y), SMBG decision-making as the predicated statistical mediator (M), and illness coherence beliefs (IPQ) as the predictor variable (X).

The first step in the predicted pathway, testing whether illness coherence beliefs (IPQ) significantly account for variance in PAID scores, controlling for education and SES level, was supported $(\mathrm{p}<.001)$. The second step in the predicted pathway, testing 
whether illness coherence beliefs (IPQ) significantly account for variance in SMBG decision-making, was supported $(\mathrm{p}=.044)$. The third step in the predicted pathway, testing whether both SMBG decision-making and illness coherence beliefs (IPQ) as a group account for variance in PAID scores was supported $(p=.004)$. The final step, testing whether the relationship between illness coherence beliefs is no longer significant after controlling for SMBG decision-making was not supported; the relationship remained significant $(\mathrm{p}=.001)$. See table 9 for a summary of regression results.

\section{Hypothesis 3}

Hypothesis 3 (H3): Beliefs reflecting greater illness controllability will be indirectly associated with lower hemoglobin Alc (by way of both higher decision-making and self-monitoring frequency).

Hypothesis 3 was tested using two separate indirect effects pathways (Baron \& Kenny, 1986) with A1c at baseline as the outcome variable (Y), SMBG decision-making as the predicated statistical mediator (M), and treatment control beliefs (IPQ) as one predictor variable $(\mathrm{X})$ and personal control beliefs (IPQ) as a separate predictor variable (X).

The first step in the first predicted pathway, testing whether personal control beliefs (IPQ) significantly account for variance in A1c, controlling for habitation status and education level at baseline, was not supported. No further relationships were tested in this indirect effects model. See table 10 for a summary of regression results.

The first step in the second predicted pathway, testing whether treatment control beliefs (IPQ) significantly predict at baseline was not supported. No further relationships were tested in this indirect effects model. See table 10 for a summary of regression results. 


\section{Hypothesis 4}

Hypothesis 4 (H4): Beliefs reflecting greater illness coherence will be indirectly associated with lower hemoglobin Alc (by way of both higher decision-making and selfmonitoring frequency).

Hypothesis 4 was tested using a indirect effects model (Baron \& Kenny, 1986) with A1c as the outcome variable (Y), SMBG decision-making as the predicated statistical mediator (M), and illness coherence beliefs (IPQ) as the predictor variable (X).

The first step in the predicted pathway, testing whether illness coherence beliefs (IPQ) significantly account for variance in A1c at baseline was not supported. No further relationships were tested in this indirect effects model. See table 11 for a summary of regression results.

\section{Supplemental Analyses}

Given the lack of support for all hypotheses, indirect effects tests were run considering all SMBG frequency testers instead of limiting the tests to the selected sample of those only reporting SMBG 5 to 7 days per week. With the expanded sample, none of the hypothesized indirect effect relationships were supported.

Indirect effects tests were also run for the reverse indirect relationships. That is, those variables previously considered outcomes $(\mathrm{Y})$ were examined for possible indirect effects on those variables previously considered predictor variables $(\mathrm{X})$ through SMBG decision-making, see Figure 6. None of the hypothesized indirect effects were supported.

Primary study variable associations. Primary study variables were examined for basic associations with one another using correlations. Items related to SMBG or personal control used a Spearman's Rho statistic due to data type or non-normal 
distribution; all other items used a Pearson's r statistic. Associations were found between a number of variables, see table 7 for correlation results.

All SMBG items were found to have a moderate to strong positive correlation with one another $(\mathrm{p}<.001)$. SMBG decision-making use was found to also be related to illness perceptions, including a positive relationship with personal control and coherence beliefs but not treatment control, and a negative relationship with both outcome variables (A1c at baseline and PAID score). SMBG frequency was not found to be correlated with additional primary variables. SMBG adherence to recommended daily frequency was found to also be weakly positively correlated with treatment control beliefs and weakly negatively correlated with PAID scores.

Illness perceptions related to treatment and personal control were shown to be moderately related to one another but only personal control was related to diabetes coherence. Personal control scores had a weak negative correlation with PAID scores. Illness coherence was shown to have a moderate negative correlation with both PAID scores and weak negative correlation with A1c at baseline.

Had primary variables been shown to be more strongly related to outcomes, whether in regression from indirect effects tests or with correlation statistics, it would have been informative to examine the primary variables within one comprehensive regression test. However, given the limited associations found, further supplemental analyses are focused on clarifying individual primary variable relationships with outcomes.

Glucose control grouping. In order to compare differences between glucose control outcomes, subjects were grouped into three categories of glucose control (A1c) 
based on ADA guidelines related to risk for disease complications (ADA, 2012). One group was comprised of those with A1c at baseline under $7 \%(n=21)$ as these are considered to be 'adequately controlled' and within a reasonable goal for glucose control for an individual with T2DM. The other group categories included a 'borderline control' group with individuals with an elevated risk of complications (under 9\%; $\mathrm{n}=62$ ) and 'very poor control' group with individuals at high risk for diabetes complications $(9 \%$ or above; $n=34)$. For these analyses, the outlier originally removed from A1c was reentered. Of the primary variables tested, only SMBG use for decision-making, tested using both nonparametric (Kruskal-Wallis) and parametric testing (ANOVA), was shown to significantly differ for each glucose control group $[\mathrm{H}(2)=9.796, \mathrm{p}=.007 ; \mathrm{F}(116)=5.50$, $\mathrm{p}=.005]$. Post hoc analyses for ANOVA (Scheffé) revealed a significant difference between the three grouping with the 'adequately controlled' group reporting significantly higher use of SMBG for decision-making than the 'very poor control' group $(p=.010)$ and the 'borderline control' group reporting significantly higher use than the 'very poor control' group ( $p=.040$; see Figure 7$)$. No significant differences were found between groups on diabetes belief scores (IPQ), diabetes distress (PAID) or SMBG adherence to provider recommendations (SDSCA).

\section{Diabetes distress and self-monitoring of blood glucose decision-making use.}

The relationship between SMBG decision-making and distress was further examined due to results from the testing of hypothesis 2. Non-parametric analysis (Kruskal-Wallis Htest) was used to examine differences in reported diabetes distress (PAID) by SMBG decision-making group. PAID scores were found to significantly differ by SMBG decision-making group: $\mathrm{H}(4)=16.226, \mathrm{p}=.003$. Post hoc analyses (Tukey HSD) revealed a 
significant difference between those responding 'a moderate amount' and 'extremely' $(\mathrm{p}=.009)$. See figure 8 for a figural representation of this relationship. 


\section{DISCUSSION}

The purpose of the present study was to examine the use of the diabetes selfmanagement strategy self-monitoring of blood glucose (SMBG) from within a selfregulatory theoretical framework. In this study, statistical analyses were used to examine the relationship between diabetes illness perceptions, SMBG behaviors, and diabetes wellness (diabetes distress and metabolic control) as components within a self-regulatory, common sense model of diabetes (Leventhal, 1970). Cross-sectional, self-report data of beliefs related to three components of illness perceptions (beliefs about the ability for treatment and personal actions to affect diabetes outcomes and the extent to which one feels diabetes makes sense to them) were examined for their connection to overall diabetes distress and overall glucose control. These relationships were considered within the context of the use of SMBG as a critical, self-regulatory, personal feedback tool. It was predicted that a higher frequency of using SMBG as a feedback tool, by way of increased use of this tool, would mediate the relationship between diabetes beliefs and these outcomes.

The study was comprised of a sample of individuals with Type 2 diabetes who were prescribed injected insulin, or daily injected diabetes medication, who were receiving treatment at a specialty clinic located in a small, Midwestern town adjacent to a mid-sized city. Participants were adults, predominantly Caucasian, were generally highly educated, and had a range of socioeconomic and employment statuses. 


\section{Hypothesis Testing}

Hypothesis 1 (H1): Beliefs reflecting greater illness controllability will be indirectly associated with less diabetes distress (by way of both increased self-monitoring decision-making and higher frequency of testing).

Hypothesis 1. The first step in the test of indirect effects test for hypothesis 1, the association between controllability beliefs and distress, was not supported. This relationship was predicted to be significant based on prior research that found associations between diabetes distress and other perceptions related to self-management.

The lack of a significant regressive relationship between personal control and distress is surprising. Personal control, or the degree to which and individual feels that their personal actions have an impact on health outcomes, is generally thought to be a similar construct to diabetes-specific efficacy, which is consistently found to be related to distress (Robertson, Amspoker, Cully, Ross \& Nail, 2013). The lack of support for this relationship may suggest that personal control beliefs are qualitatively different from diabetes efficacy beliefs. Examination of the phrasing of personal control items in the IPQ reveals that they are passively worded (e.g. "there is a lot which I can do to control my symptoms") rather than actively worded (e.g. "I do a lot to control my symptoms"). It may be that personal control beliefs, as measured by the IPQ, represent a belief that control is possible, but without a consideration for one's personal ability to act on this belief, as in self-efficacy. Diabetes self-efficacy was not measured in the present study; comparisons were not possible.

A lack of a relationship between treatment control and distress may be due to conceptual concerns about the subscale. Beliefs about treatment control, or the degree to 
which an individual feels that their recommended diabetes treatment regimens have an impact on health outcomes, had the most missing data of all primary variables. The single item missed or skipped by the most participants $(n=4)$ on this subscale was 'my treatment will be effective in curing my diabetes.' There was a range of responses for this item with the modal response as 'neither agree nor disagree.' It may be that the use of the word 'cure' in relation to diabetes was puzzling to respondents, leading them to skip the item. These respondents would not have been included in analyses. Interestingly, many responded that they "strongly" believed that treatment can cure their diabetes, a belief that could be considered to be inflated or unfounded as there is no known cure for T2DM once diagnosed. However, the implications of the "strongly" response depend on the reader's interpretation of the word cure, which cannot be known given the questionnaire format. It may be that a strong belief in a diabetes cure combined with a lack of improvement in ones own condition could be disheartening and lead to difficulty coping and increased diabetes distress. Conversely, a strong belief in controllability through treatment may be related to less distress due to reduced concern for complications or one's ability to manage T2DM. A bidirectional relationship such as this has the potential to mask any linear relationship between the two variables.

It may also be that the relative homogeneity of the sample, as it relates to the construct of controllability, led to conceptual problems with comparing response differences. A weak, negative correlation was found between personal control beliefs and distress in supplemental analyses, suggesting that a sample with more varied beliefs related to their personal ability to control diabetes might have had a stronger relationship with overall distress. Personal control beliefs had a significant negative skew with most 
reporting high perceived personal control. Similarly, treatment control beliefs were generally high in this sample. In essence, no respondents reported that they felt their actions, or the effect of their treatments, had no impact on their symptoms or diabetes management. Though a transformation was performed to reduce skew, the relative homogeneity of the sample in reported beliefs about controllability may have resulted in conceptual concerns that extend beyond problems with data normality. These concerns make it very challenging to understand, either conceptually or statistically, any existing relationship between these constructs.

Hypothesis 2 (H2): Beliefs reflecting greater illness coherence will be indirectly associated with less diabetes distress (by way of both increased self-monitoring decisionmaking and higher frequency of testing).

Hypothesis 2. The first step in Hypothesis 2, that coherence is related to diabetes distress by way of decision-making use of SMBG, was not supported. However, greater perceived coherence, or understanding, of T2DM was related to less diabetes distress and this relationship was still significant when controlling for SMBG decision-making use. Instead of the hypothesized indirect effect, with SMBG decision-making mediating the relationship, the results suggest a possible indirect effect of beliefs between SMBG decision-making and distress. That is, an observed significant relationship between SMBG decision-making and diabetes distress was no longer significant when controlling for illness coherence. This relationship was further examined in supplemental analyses. A figural representation of diabetes distress (mean PAID scores) for responses related to the extent to which an individual is using SMBG for decision-making shows an interesting pattern (see figure 7). Average reported distress for individuals at varying levels of the 
use of SMBG for decision-making appears to follow a curvilinear pattern with those falling in the middle of the SMBG decision-making range experiencing the greatest average distress. Mean distress scores for participants in the middle range fell above one standard deviation from mean for the whole sample indicating significant distress. Nonparametric tests found the middle range group to significantly differ from those reporting their degree of SMBG decision-making use as "extremely." Though responders in the lower SMBG decision-making groups ("not at all' or "somewhat") were not shown to significantly differ from others, this may be due to a loss of power with follow-up analysis, or the small group size of those using less frequently using SMBG for decisionmaking. With increased sample size, it may be that the pattern appears more linear rather than curved. It may also be that participants reporting SMBG decision-making to a lesser degree are reporting lower diabetes distress due to the impact of another factor not considered in the present study, such as their perceptions about the importance of tight glucose control.

Hypothesis 3 (H3): Beliefs reflecting greater illness controllability will be indirectly associated with lower hemoglobin Alc (by way of both higher decision-making and self-monitoring frequency).

Hypothesis 3. The first step in the test of the indirect effects test for hypothesis 3, the association between controllability beliefs and glucose control, was not supported. This relationship was predicted to be significant based on prior research that found associations between control beliefs and glycemic control.

The lack of a significant relationship between personal control and glycemic control is inconsistent with a body of prior research that has consistently found such an 
association (McSharry et al., 2011). It also differs from what might be expected given prior research supporting a relationship between efficacy and glycemic control (Zulman et al., 2012) though, as mentioned previously, personal control beliefs as measured by the IPQ are likely measuring a construct distinct from diabetes self-efficacy.

It is also interesting that treatment control was not related to actual glucose control. It was previously thought that treatment control has not been consistently supported in the literature, as relates to glycemic control, due to a lack of specification of treatment types (Mc Sharry et al., 2011), such as those using insulin and/or oral medications or diet alone; however, the present sample are all using insulin or daily injected diabetes medication, significantly minimizing this problem. It may be that the overall poor health of this sample, as evidenced by significant medical complications and poor glycemic control, makes testing associations with glycemic control difficult. Or, as mentioned previously, that an overall high belief in treatment controllability make associations difficult to observe.

Another possible reason for a lack of relationship between treatment control and glucose control is that some individuals have changes in their treatment control beliefs across time, which are impacted by not perceiving improvement in glucose control. Insulin treatment in T2DM is generally seen as a last resort for management of glucose levels when oral medications or diet alone have not been effective. As observed in this sample, when someone has been on insulin for a longer period of time, they are less likely to believe in the ability of treatments, in this case insulin, to control their diabetes. Therefore, for some individuals, a higher glucose level may be related to lower treatment beliefs while for others it is related to higher treatment beliefs. 
Hypothesis 4 (H4): Beliefs reflecting greater illness coherence will be indirectly associated with lower hemoglobin Alc (by way of both higher decision-making and selfmonitoring frequency).

Hypothesis 4. The first step in the test of indirect effects test for hypotheses 4, the association between diabetes coherence and glucose control, was not supported. There is limited prior research on diabetes coherence and outcomes. The hypothesis was based on prior research consistently finding associations between general diabetes knowledge and glucose control. Coherence was found to be related to glucose control via basic correlation. It is possible the relationship between these variables is complex and there was not sufficient power in the present study to support a regressive relationship.

\section{Support for the Self-Regulation Model for SMBG}

Though there is extensive prior research on SMBG behaviors in the T2DM population, little prior research has yet to systematically examine the use of SMBG for decision-making as it relates to diabetes outcomes, and no known studies have examined its use from within a theoretical framework. Much of the prior SMBG research has been frequency driven. The present study sought to further the current state of knowledge about SMBG behaviors by drawing from the broader health psychology literature on the psychological processes that explain self-management choices.

Due to the lack of prior, theoretically-based SMBG research, only select portions of the Self-Regulation Model (SRM) were examined, in an exploratory fashion. Specific illness perceptions, those related to controllability and coherence, were examined individually. Controllability has some prior support as it relates to overall diabetes care and coherence with no prior studies related to SMBG decision-making. Coherence has 
not yet been examined as it relates to SMBG.

In the present study, the planned methods used to test the self-regulation model for SMBG did not yield significant results. As such, results are inconclusive related to the usefulness of the Self-Regulation, Common Sense, Model of illness beliefs as it relates to the use of SMBG for diabetes management. However, findings point to a broader possibility that the Self-Regulation Model may still be an appropriate way to conceptualize SMBG use. For example, a finding from supplemental analyses indicated that A1c was not associated with SMBG adherence, but was associated with SMBG decision-making. This supports the idea that use as a decision-making strategy may be critically distinct from simply examining SMBG frequency adherence in glucose control efforts, which has implications for both clinical practice targets and future research. Additionally, diabetes coherence was shown to be related to diabetes distress with some effect on decision-making. It may be that improvements in glucose control efforts by way of increased decision-making use of SMBG depend on the degree of illness coherence perceived by the individual. Further research is needed to examine the nature of the relationships between illness beliefs, SMBG behaviors and diabetes outcomes.

A number of aspects of the study characteristics may have influenced the nature of the participant sample and, in turn, characteristics of the sample may have influenced the observed study findings. These issues are discussed below.

Study characteristics influencing participation. The appointment format structure for blood draws at the JDC made it difficult for research staff to be present at the clinic at the time that each eligible patient presented for their blood draw. At the JDC, blood draw appointments are scheduled for a given date, but may occur as a 'drop-in' 
within a time window of several hours. In addition, because the screenings were completed prior to JDC appointment reminder calls, in some cases, patients were rescheduled which resulted in them presenting for their blood draws on different days than those anticipated by the research study personnel.

The point of recruitment proved difficult for research staff with a high number of eligible patients declining to participate due to a lack of time. Several, particularly those presenting to the clinic very early in the morning (e.g. 7:00 am), planned to go directly to work after their appointment and were unable to spare the time to discuss consenting. In addition, most patients are required to be fasting for blood draw appointments and were hungry; taking the time to review study details and consent procedures may have been deemed too uncomfortable or difficult, leading many to decline.

Completion rates were similar to a previous JDC study; however, rates were lower than expected due to the added lottery incentive. Approximately one quarter of participants did not return the questionnaire. Return rates appeared to improve when each participant was provided with a stamped return envelope at the point of consent, rather than having it offered as optional. It is likely that a number of participants forgot to bring their questionnaires to their provider appointment and did not wish to return the questionnaire to the JDC at a later date. Of those who completed questionnaires, missing data was around $20 \%$, as predicted.

Revocations were rare (4.3\%) and several appeared to be accidental. Some participants returned the revocation form in the same packet as the completed questionnaire. Others noted as their reason for revocation that they did "not have time," despite having completed all necessary actions, suggesting that they were referring to a 
follow-up study that was described during consent. A small number, due to screening error, were found to be ineligible at the point of medical chart data review.

\section{Participant characteristics with potential influence on outcomes}

High disease comorbidity. Most participants had glucose control levels (A1c) that fell above the ADA recommended target for glucose control. A1c levels above this target represent a higher likelihood of experiencing cardiovascular and other diabetes-related complications and morbidity. In addition, the sample had a mean BMI in the obese range and was within one standard deviation of morbid obesity - a health marker that is also highly related to significant medical comorbidity. Not surprisingly, the sample had a high number of comorbid medical diagnoses in their medical charts; the most common of these were hyperlipidemia, hypertension, and peripheral neuropathy. Given the high comorbidity rate, the participant sample from this study is less likely to see quick progress with A1c improvements when compared with other T2DM participant samples. As such, these participants may have different perceptions of treatment efficacy in their diabetes management efforts.

High burden of care. Chart-extracted provider diabetes self-care recommendations for the sample were generally intensive. The modal prescribed times per day of injecting insulin was 4 with modal SMBG recommendations of 3-4 times per day. This translates to a large portion of the sample having recommendations to use a needle for either testing or injecting around 8 times per day. In addition, many participants had a high number of medications prescribed, including diabetes oral medications (around 75\%), further contributing a high self-care burden. 
Time since diagnosis. There was a large range of experience with T2DM and insulin with some as recently diagnosed 2 years ago and others having a T2DM diagnosis for over 40 years. Mean insulin prescription onset occurred about 6 years prior to the study. Both variables have missing data due to no record of the information in existing medical charts. This was most often the case for patients with electronic medical records, as old medical records were not always uploaded to the electronic system (around one third of patients). As such, associations between variables should be interpreted with caution.

High overall compliance in multiple self-management domains. Due to the impact of health behaviors on glucose management, such as dietary and physical activity behaviors, T2DM self-care recommendations generally fall in a number of health domains (ADA, 2012). In this study, these other health domains were measured by responses on a self-report measure of days engaging in recommended self-care activities during the week prior. A number of associations were found between primary study variables and dietary behaviors, such as following a healthful eating plan and spacing carbohydrates throughout the day, but there were fewer associations with exercise behaviors and medication behaviors. This may be due to the non-normally distributed data from these variables that is difficult to interpret. Only around $15 \%$ of respondents reported that they did not take their recommended diabetes medication on at least one day during the last week. Similarly, only $24 \%$ reported not taking insulin as recommended every day. These responses reflect a sample with high overall compliance, limiting interpretive possibilities. Non-parametric tests revealed some associations in the skewed 
variables, though these should be interpreted with caution. For example, those reporting higher illness coherence were more likely to take insulin injections as recommended.

Overall low physical activity. Responses on exercise behavior items reflected unusually low compliance. The modal response for both items, which were related to general levels of activity and planned exercise, was 0 days per week. While this sample appears to be highly compliant with medication recommendations, they also appear to be largely sedentary. This may be partly related to the older mean age of the sample or the high rate of physiological comorbidity, and is reflected in the sample's high mean BMI. Exercise is considered by the medical community to be a means for improved glucose control through both direct and indirect pathways. Directly, aerobic activity improves the body's ability to process glucose. Indirectly, resultant weight loss can decrease burden on endocrine system function, thus, improve glucose processing. The pattern of responding in this sample highlights the complexity of adherence to recommendations for these participants. That is, health decisions related to one domain of care are not necessarily indicative of health decisions in other domains. Self-regulatory processes may greatly differ between self-care domains based on beliefs about the importance of these behaviors in controlling diabetes, or other, external barriers such as pain.

Diet behaviors varied. Participants showed greater variability on responses related to diet plans. These variables were associated with many primary items suggesting that diet behaviors are more open to the influences of perceptions and decision-making than other self-care behaviors. Interestingly, following a healthful eating plan, while associated with all other primary variables, was not associated with treatment control beliefs. It is possible that a healthful eating plan is not viewed as a medical 
treatment, though diet is used to treat T2DM, but rather a self-care mechanism of personal action. This has important implications for understanding overall perceptions of T2DM management self-care activities.

Mental health. While it would be interesting to examine illness perceptions and behaviors as they relate to psychological comorbidities, only a small number of participants were found to be diagnosed with a mood or anxiety disorder via a review of medical chart data. This number likely greatly underestimates the existence of mental health diagnoses in the present sample. Recent estimates of clinical depression rates in individuals with T2DM are around two times greater than that of the non-T2DM population (Gonzalez et al., 2007), which is thought to be due to both physiological factors and self-care burden (Renn et al., 2011). The low number of noted psychological diagnosis in this sample is possibly due to the nature of the JDC. Specifically, the JDC keeps their own medical records separate from other clinics for the approximately $2 / 3$ of patients who have paper medical charts, and there is an absence of a mental health practitioner within the clinic setting. Mental health diagnoses were likely only noted in the chart if the participant were prescribed psychotropic medications or if the patient brought records from another facility where they were evaluated for psychological conditions. As such, some participants may have an undiagnosed mental health disorder.

Nearly $30 \%$ of participants were found to be prescribed an antidepressant medication. Unfortunately, antidepressant prescription cannot serve as a proxy for mental health diagnosis in T2DM, as medications from this class (e.g. gabapentin, pregabalin, topiramate, tegretol) are frequently prescribed as an effective method for the management 
of nerve pain, such as the pain associated with peripheral neuropathy, a common complication of T2DM.

\section{Decision-Making as a Critical Distinction in SMBG Behavior}

To date, very little research has considered decision-making separately from other SMBG behaviors. Earlier studies have supported a relationship between greater knowledge about strategies for glucose management and better control (Brega et al., 2012; Zulman et al., 2012) but these were not specific to SMBG. The present study is unique in considering decision-making separately, as a specific strategy among a set of other SMBG behaviors that are more typically studied. Though all SMBG behaviors were shown to be moderately interrelated via correlation statistics, their associations with other primary variables differed. This suggests that these differing behaviors may correspond to distinct concepts. For example, SMBG frequency of adherence to daily provider recommendations was associated with beliefs about treatment control only, and not with personal control or coherence beliefs. For SMBG decision-making the opposite was true. Given these associations, it is possible that SMBG adherence behaviors are related to specific beliefs about the helpfulness of medical intervention, while SMBG decisionmaking reflects a more complex system of beliefs and behaviors about engaging in what makes sense to an individual given their personal ability to affect outcomes.

Interestingly, in this sample, SMBG adherence was not related to actual glucose control and SMBG decision-making was, though both SMBG behaviors were negatively associated with problems coping with diabetes (PAID). This suggests that decisionmaking represents a construct with more direct influence on outcomes, and is therefore an important consideration in the self-management practices of individuals with T2DM. 
Indeed, national recommendations for diabetes management have been updated since the beginning of the present study to address the importance of this practice in recommendations for SMBG. However, due to limited research, the evidence for these changes was classified as based on "expert consensus" (ADA, 2013, pg s17).

\section{Coherence is Key}

Illness coherence perceptions, or the extent to which individuals feel they understand their T2DM, were more varied than controllability beliefs. Though the sample was generally supportive of management practices as important, very few (about 12\%) responded in a way that indicated they had no uncertainty about, or difficulty understanding, their T2DM. That many feel they do not understand their T2DM is particularly interesting given the relative lack of prior research regarding this construct. It was previously thought that coherence would be lower in individuals newly diagnosed with T2DM or who have not completed diabetes education but improves with education. In this study, completed within a specialty clinic where nearly all participants have already completed some degree of diabetes education, coherence was not associated with time since T2DM diagnosis or time since insulin prescription. The large range in coherence beliefs in a generally treatment-compliant, experienced T2DM population might dispose coherence to be an illness belief particularly open to intervention. For many, there is room for improvement in coherence while other factors appear to have been targeted in either diabetes education, provider contacts, or both as evidenced by high overall controllability beliefs. In this sample, greater coherence was associated with a higher level of education completed and higher socioeconomic status. As coherence was also associated with less distress and better glucose control, education level may be 
an important consideration for tailoring interventions targeted improving self-care decision-making through T2DM coherence.

\section{Distress is Not Related to Glucose Control}

Participants reported a range of diabetes distress with some endorsing little to no difficulty and others endorsing very frequent problems with things such as worrying about complications and feeling overwhelmed, angry, alone, deprived, or unsupported in their diabetes management efforts. Many individuals reported some level of distress, which is not surprising given the high burden of treatment with insulin prescriptions and the numerous recommended self-care behaviors in other areas of their life. The degree of distress in this sample was relatively matched to a normative sample of individuals with T2DM also on insulin treatment. Those with less distress were generally younger, with high personal control beliefs and high coherence beliefs and were generally using SMBG for decision-making.

There is limited prior research related to the relationship between diabetes distress and SMBG decision-making. Though none of the hypothesized relationships were supported, distress was found to be negatively related to illness beliefs and SMBG behaviors, including decision-making, through basic correlation. Additionally, distress was not shown to be related to overall glucose control through basic correlation.

In the present study, participants completed self-report measures, including items related to diabetes distress, before receiving their A1c results. This allowed for the sample's perceptions of control to depend exclusively on their SMBG results. Diabetes distress was not shown to be directly related to A1c. Though the lack of a relationship may be, in part, due to not being aware of their results, those engaging in SMBG 
generally have an estimate of their control based on SMBG results. The lack of a relationship between distress and glucose control highlights that effective coping related to diabetes may depend more heavily on extraneous factors.

\section{Treatment Control Beliefs and Time Since Diagnosis}

Treatment control beliefs were found to be negatively correlated with time since insulin prescription, suggesting that those who have been on insulin longer are less likely to think that treatment measures can significantly help to manage or their T2DM. This might be reflective of an individual who has been on insulin for a long time with very limited improvements in their glucose control. Indeed, prior research has pointed to a possible deterioration in treatment control beliefs when patients made lifestyle changes, such as beginning insulin treatment based on provider recommendations but not observing improvements in their condition, leading to lower adherence to recommendations in the future (Lawton et al., 2008; as cited in Gomersall et al., 2010).

\section{Personal Control and Treatment Control Beliefs Differ}

Association with other variables, such as diet behaviors, differ between personal and treatment control beliefs, suggesting that participants view some self-care behaviors, such as diet, in a personal control category and others, such as medications, as a medical treatment. Interestingly, a relationship to SMBG decision-making was generally seen to exist across both categories. SMBG is, perhaps, seen as both a tool to aid personal control and also aid treatment. These views may also differ by the individual.

Treatment control was associated with fewer reported problems related to diabetes distress, greater belief that personal behaviors impact diabetes outcomes, and greater adherence to provider SMBG and diabetes oral medication recommendations. 
In this sample, both treatment and personal control beliefs were not associated with glucose control outcomes, suggesting that illness beliefs alone do not explain why some individuals are more successful at managing their diabetes than others.

Other items on the treatment control beliefs measures are worded in a very general manner. For example, one item asks the participant to identify how strongly they feel that "there is nothing that can be done to control my diabetes" without specifying a treatment type, such as medications or insulin. It may be difficult to interpret what is measured by this subscale as it relates to the T2DM population on insulin.

\section{Strengths and Limitations}

\section{Strengths of the Present Study}

Contribution to limited literature. The present study represents a significant contribution to the current body of literature on diabetes self-management; there is little prior research examining SMBG that considers whether or not the individual is using their result to inform health behaviors. This is despite a recent adjustment to medical guidelines from a major national organization (ADA) reflecting the importance of this construct. Current research on SMBG decision-making is very limited and no known studies have been designed with a theoretical framework for understanding for this relationship. Without an understanding of the mechanism through which this activity improves control, informed strategies to improve SMBG practices in individuals with poor control will be limited.

Within the context of limited research on SMBG decision-making the relationship between diabetes distress and SMBG decision-making has been particularly overlooked. The present study represents a significant contribution to the literature in its consideration 
of diabetes distress as an important factor in understanding the complex psychological processes involved in the use of SMBG in this manner.

Mixed data sources. The present study utilized both medical chart data and selfreport information. Often, studies examining perceptions do not have the support of medical chart data. The collective use of both self-report and medical chart data allows a more comprehensive analysis of participant health status and medical care recommendations and reduces bias due to self-reporting.

The sample is prescribed insulin. Prior research examining diabetes perceptions in the T2DM population rarely exclusively examine an insulin-using population. The inclusion criteria requiring an insulin prescription, or other daily injected diabetes medication, ensures that all participants share a similar degree of burden that is typically much higher than those not prescribed an insulin regimen. Indeed, the typical participant in the present study is recommended to use insulin and SMBG around 4 times per day for each activity in addition to the comprehensive self-care regimen related to many other areas of their daily life. This high degree of burden makes the use of self-regulatory strategies particularly relevant for all participants in the study.

The sample is free from recent clinic setting feedback. The decision to arrange the study such that completion of the questionnaire packet took place before a provider visit allowed the perceptions reported in the questionnaires by participants to be based almost entirely on feedback experienced outside of the clinic setting, such as from SMBG or self-perceived symptoms. This ensured that participant perceptions related to the illness regulation process, as they have occurred over the past several weeks, were due to self guided actions rather than medically guided actions. In addition, glucose control 
averages for the weeks prior to the appointment, likely the same time period on which the participant based their responses, were collected within the same week that the questionnaires were completed. This provided a physiological indicator of actual glucose control to be used in the study as an outcome with the reported perceptions of glucose control occurring prior to the participant being aware of this result. It is difficult in any illness requiring intensive medical intervention to capture patient perceptions without recent influence of provider feedback, particularly when recruitment for studies typically occurs at the point of the provider visit.

\section{Limitations of the Present Study}

Cross-sectional design. A primary limitation of the present study is the crosssectional study design. The basic indirect effects model used in this study (Baron \& Kenny, 1986) has been criticized for considering indirect effects, referred to as statistical mediation, to be analogous to the construct of mediation, which in theory requires temporal data. One way to remedy this would be to conduct a longitudinal study that follows glucose control over time. This may be problematic for glucose control, however, as change in A1c is likely to be minimal for those already exhibiting control close to recommended levels. For the present study, indirect effect findings should be considered correlational.

Homogenous sample. Another limitation in this study is the relative homogeneity of the sample. The sample is all from a specialty clinic, nearly all had had some amount of formal diabetes education and most are testing at least daily. From a conceptual standpoint, insulin users who chose to attend a specialty clinic may be different from community settings of poorly controlled Type $2 \mathrm{DM}$. Indeed, this is reflected in 
participant responses related not only to high adherence to SMBG recommended frequency but also in that most reported that they believed personal behaviors played a large role in the outcomes from their diabetes. The limited range in these constructs may have reduced the impact of these and other constructs examined in the study.

It is possible that the greater number of significant relationships between coherence and other primary constructs is due to the comparatively greater range of beliefs related to illness coherence in this sample. With a more varied range of beliefs, it is easier to measure differences in other constructs as they relate to the belief. It may be that with a sample with more varied controllability beliefs, such as may be found in a community-based or primary care clinic, relationships will be more easily examined.

Measurement of SMBG. One of the most critical data limitations in this study is the type of measure available to examine SMBG behaviors, including SMBG decisionmaking. Decision-making was measured with a single likert-type item, leading to challenges identifying appropriate testing procedures and significant difficulty interpreting results. Unfortunately, given the lack of prior research related to this construct, there is not currently a validated measure of SMBG decision-making available to use.

Additionally, the measure used for other SMBG behaviors, the SDSCA, was problematic. Though the SDSCA is generally considered a gold-standard for measuring SMBG and other self-care activities in T2DM, the unique subset in this study, those prescribed insulin therapies, have SMBG frequency recommendations that greatly exceed general recommendations. The difference in frequency recommendations led to the problematic ceiling effect that was observed in our sample and rendered the use of the 
SMBG frequency variable in the original planned analyses to be highly problematic. None of the hypothesized relationships between illness beliefs, SMBG decision-making behaviors and diabetes distress or glucose control were fully statistically supported. It may be that the loss of power due to excluding participants who report testing less than 5 days per week impacted the likelihood of finding a significant result.

\section{Implications and Recommendations}

Clinical implications and recommendations. Further research is needed before clear clinical implications can be drawn. Much of the research related to SMBG is atheoretical and no known studies examining SMBG decision-making are based on a conceptual framework from which to inform clinical practice. Without an understanding of the mechanism through which decision-making improves control, recommendations for strategies to improve SMBG practices in individuals with poor control are limited.

However, there are a number of patterns observed in the present, exploratory study that may help to shape the direction of future clinical intervention and examination. Results from the present study point to possible clinical targets such as illness coherence and diabetes distress. Findings suggest that illness coherence may support the use of SMBG for decision-making. This has implications for interventions targeted at improving coping with diabetes demands. Coherence was found to differ by education level and SES and greatly vary in an otherwise relatively homogenous sample. If supported in future studies, providing diabetes coherence-specific education for individuals with low education could facilitate improvements in perceived distress and the possible impact of distress on health outcomes. Alternatively, providing supportive materials to support 
coherence needs, such as tables or decision-trees may prove helpful in reducing distress or improving glucose control.

\section{Research implications and recommendations}

Measurement of SMBG. Significant statistical and conceptual interpretation difficulty resulted from problems with SMBG variables in this study. Results suggest that the SDSCA may not be an appropriate measure of SMBG frequency in an insulin treated population. Current recommendations for intensive insulin users are to SMBG at least with every meal or insulin administration (ADA, 2013). The current study's participants were considered to be generally highly compliant due to most reporting at least daily SMBG; however, in line with medical guidelines, SMBG recommendations were generally multiple times per day. Due to the response options on the SDSCA, an individual reporting that they engaged in SMBG 7 times in the last week has the same response selection as an individual who SMBG 28 times in the last week. Though it may be that only the latter participant is strictly adhering to their provider's recommendations, this is not clear from the measure. Importantly, more robust and validated measures are needed to effectively examine SMBG decision-making. A single, likert-type item is difficult to examine both conceptually and statistically. Valid, reliable measures are needed to support future research related to this construct.

Alternative Methods. Distress was negatively related to both SMBG behaviors and illness beliefs in the present study. Due to the exploratory nature of the present study, distress was generally considered as an outcome; however, within the self-regulatory framework, distress could be understood as coping, which may act as a potential moderator between beliefs, behaviors and glucose control outcomes. Future studies could 
examine this potential relationship, ideally with a measure of depression included to control for the effects of broad depressive symptoms.

Sample. Homogeneity of the present sample, though a strength in many ways, made interpretation of results, such as those related to controllability beliefs, difficult to interpret. This was largely due to the ceiling and flooring effects from the measures selected for this study. Future studies of insulin-treated T2DM should consider utilizing different measures with expanded options to better assess for differences in this population. Alternatively, examining these relationships in a less homogeneous sample, such as a community outpatient clinic, would potentially provide a greater range of controllability beliefs. This, too, would be helpful in clarifying this construct as it relates to other components within a self-regulatory process.

\section{Summary of conclusions}

The present study examined SMBG as part of a self-regulatory process of health decision-making that involves a complex interaction of feedback, illness perceptions, and distress. Findings suggest that illness perceptions play an important role in the process of SMBG use for decision-making as it relates to glucose control and diabetes distress in insulin-treated T2DM. There are no other known studies that have examined SMBG use as a decision-making tool from a theoretical perspective. Though the study was crosssectional and largely exploratory, the study consists of both self-report and medical chart data with temporal control for feedback about overall glucose control making this a strong contribution to the current literature and providing a solid empirical foundation for future research related to SMBG as a decision-making strategy. 
Table 1. Statistical procedures for testing hypotheses with the Joint Significance Test

\section{Hypothesis 1 (H1):}

The following statistical hypotheses will be tested

(1) $\mathrm{SMBGdm}=\beta_{01}+\beta_{1}$ (controllability) $+\varepsilon_{1}$

(2) SMBGf $=\beta_{02}+\beta_{2}($ SMBGdm $)+\beta_{3}$ (controllability) $+\varepsilon_{2}$

(3) PAIDt $=\beta_{03}+\beta_{4}($ controllability $)+\beta_{5}($ SMBGf $)+\beta_{6}($ SMBGdm $)+\varepsilon_{3}$

\section{Hypothesis 2 (H2):}

The following statistical hypotheses will be tested

(1) $\mathrm{SMBGdm}=\beta_{01}+\beta_{1}$ (controllability) $+\varepsilon_{1}$

(2) SMBGf $=\beta_{02}+\beta_{2}($ SMBGdm $)+\beta_{3}$ (controllability) $+\varepsilon_{2}$

(3) $\mathrm{HbA1c}=\beta_{03}+\beta_{4}($ controllability $)+\beta_{5}(\mathrm{SMBGf})+\beta_{6}(\mathrm{SMBGdm})+\varepsilon_{3}$

\section{Hypothesis 3 (H3):}

The following statistical hypotheses will be tested

(1) $\mathrm{SMBGdm}=\beta_{01}+\beta_{1}$ (coherence) $+\varepsilon_{1}$

(2) SMBGf $=\beta_{02}+\beta_{2}(\mathrm{SMBGdm})+\beta_{3}$ (coherence) $+\varepsilon_{2}$

(3) PAIDt $=\beta_{03}+\beta_{4}($ coherence $)+\beta_{5}(\mathrm{SMBGf})+\beta_{6}(\mathrm{SMBGdm})+\varepsilon_{3}$

\section{Hypothesis 4 (H4):}

The following statistical hypotheses will be tested

(1) $\mathrm{SMBGdm}=\beta_{01}+\beta_{1}($ coherence $)+\varepsilon_{1}$

(2) $\mathrm{SMBGf}=\beta_{02}+\beta_{2}(\mathrm{SMBGdm})+\beta_{3}$ (coherence) $+\varepsilon_{2}$

(3) $\mathrm{HbA1c}=\beta_{03}+\beta_{4}($ coherence $)+\beta_{5}(\mathrm{SMBGf})+\beta_{6}(\mathrm{SMBGdm})+\varepsilon_{3}$

Path for rejecting the null hypothesis in joint significance test:

Reject if:

and... (5)

and... (6)

$$
\begin{aligned}
& \left(\left|\mathrm{b}_{1}\right| \mathrm{s}_{\mathrm{b} 1}\right)>\mathrm{t}_{.975(\mathrm{n}-2)} \\
& \left(\left|\mathrm{b}_{2}\right| / \mathrm{s}_{\mathrm{b} 2}\right)>\mathrm{t}_{.975(\mathrm{n}-3)} \\
& \left(\left|\mathrm{b}_{3}\right| / \mathrm{s}_{\mathrm{b} 3}\right)>\mathrm{t}_{.975(\mathrm{n}-4)}
\end{aligned}
$$

*SMBGdm = self-monitoring of blood glucose decision-making; SMBGf = self-monitoring of blood glucose frequency; Controllability $=$ the controllability subscale of the IPQ-R; Coherence $=$ the coherence subscale of the IPQ-R; PAIDt = total score on the Problem Areas in Diabetes Scale; HbA1c

$=$ hemoglobin A1c

$* * b_{\mathrm{x}}$ is the beta weight of a given test of regression, $\mathrm{s}_{\mathrm{bx}}$ is the standard error of the regression 


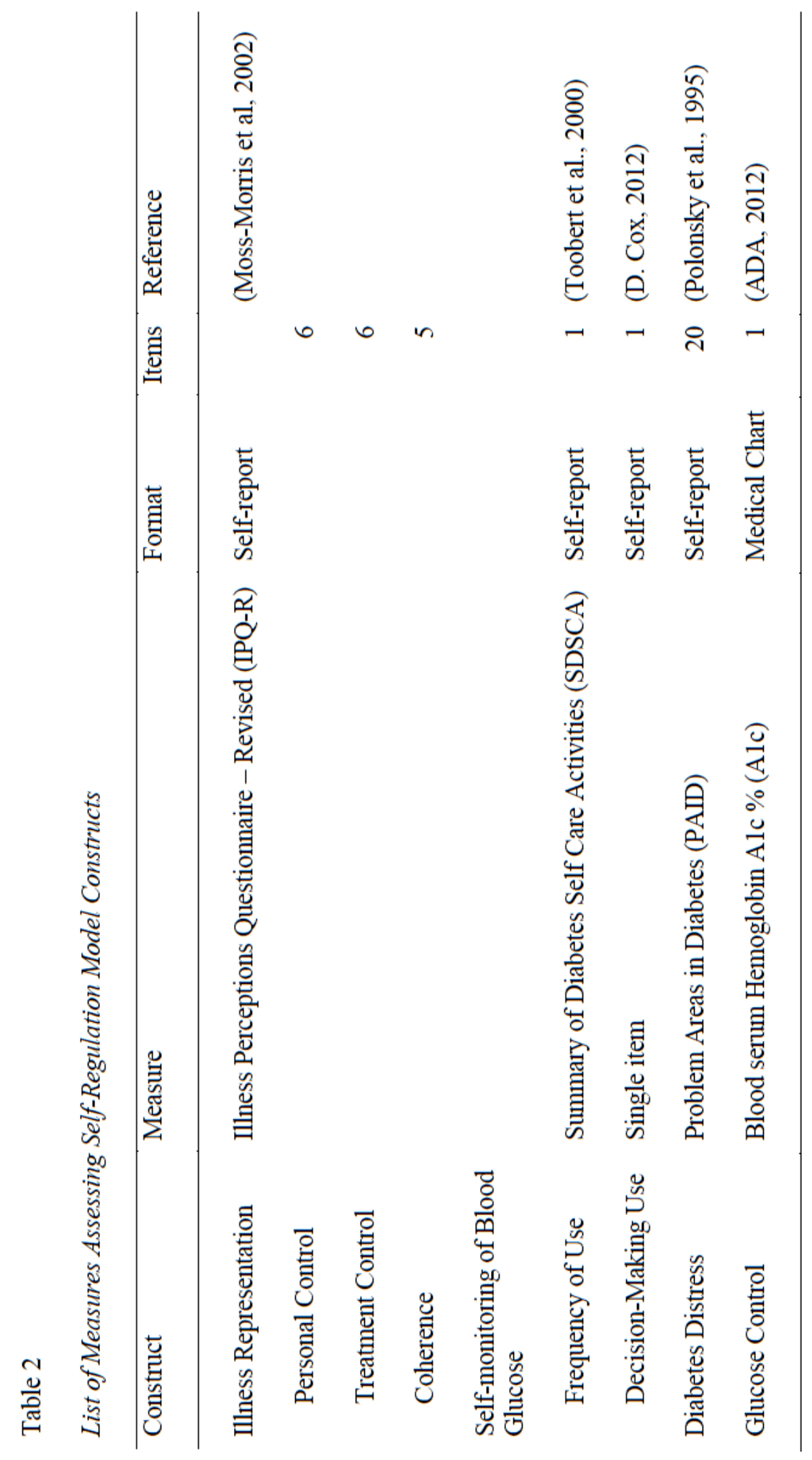




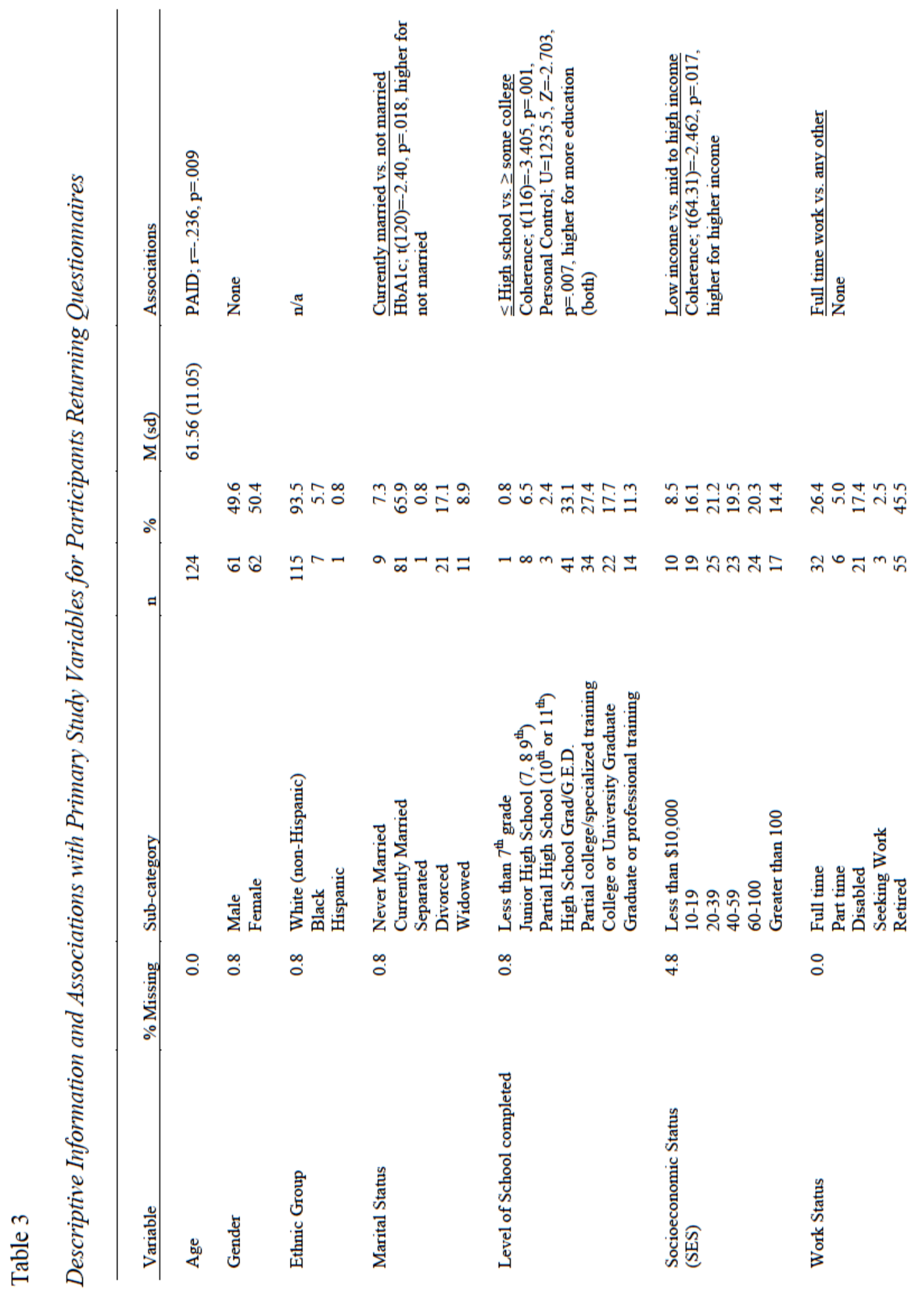




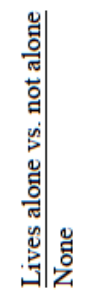

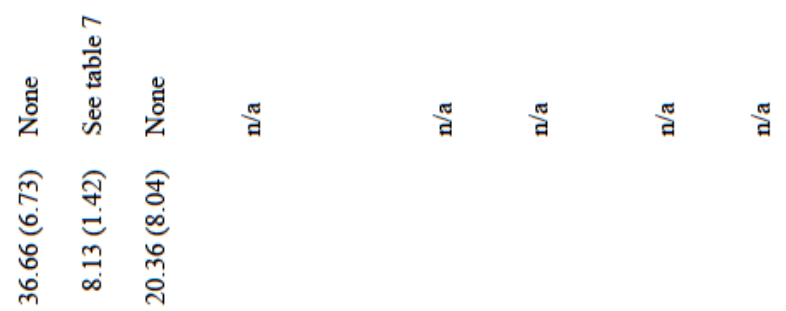

m.

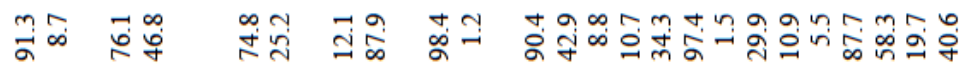

+ สำก

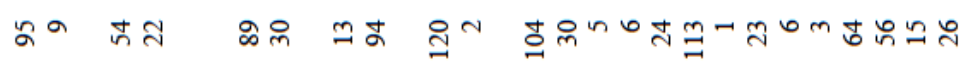
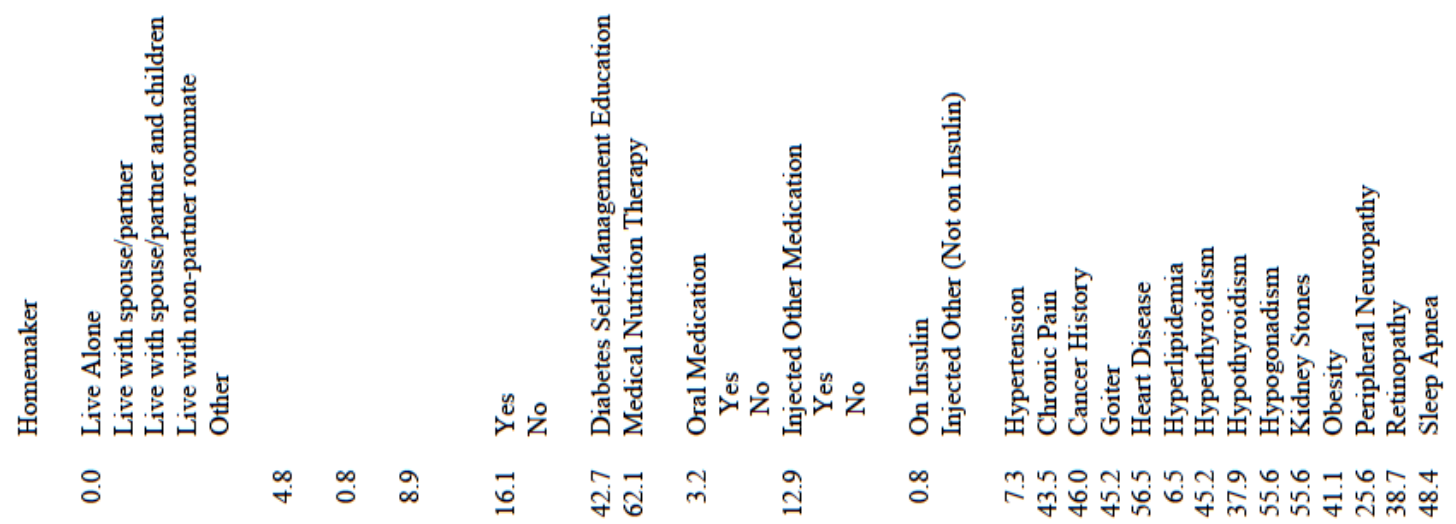

总

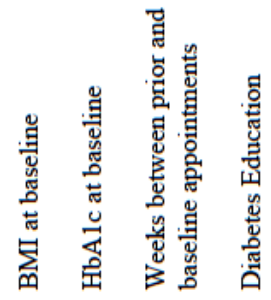

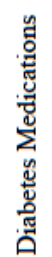

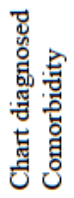




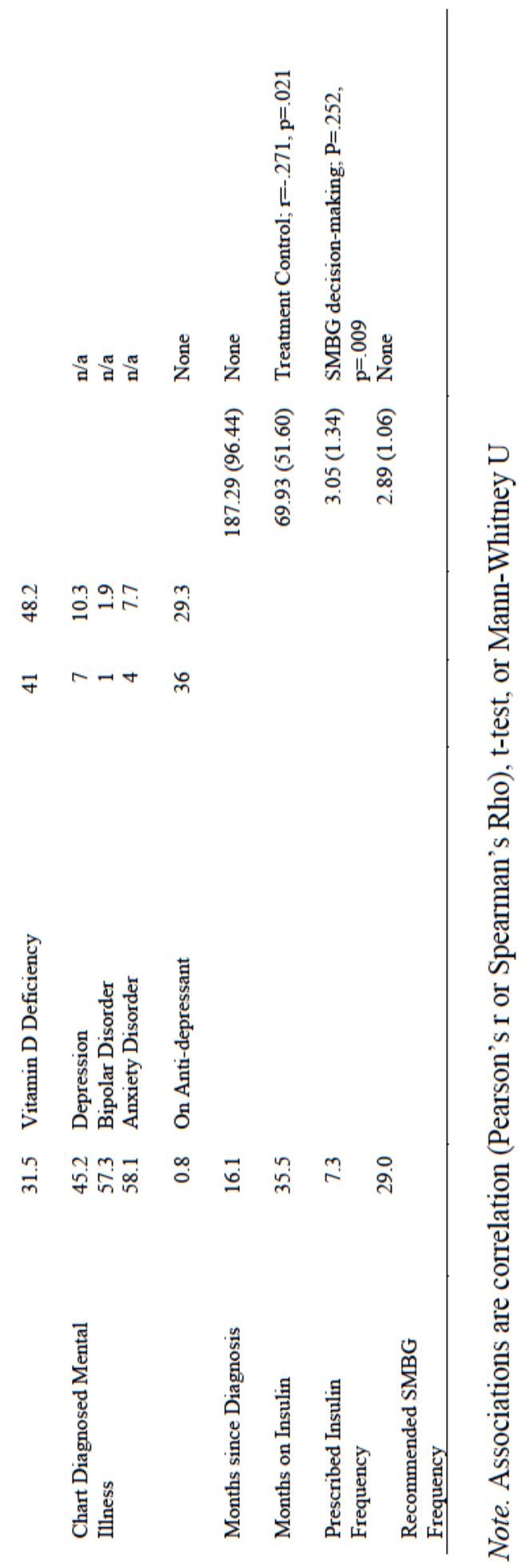




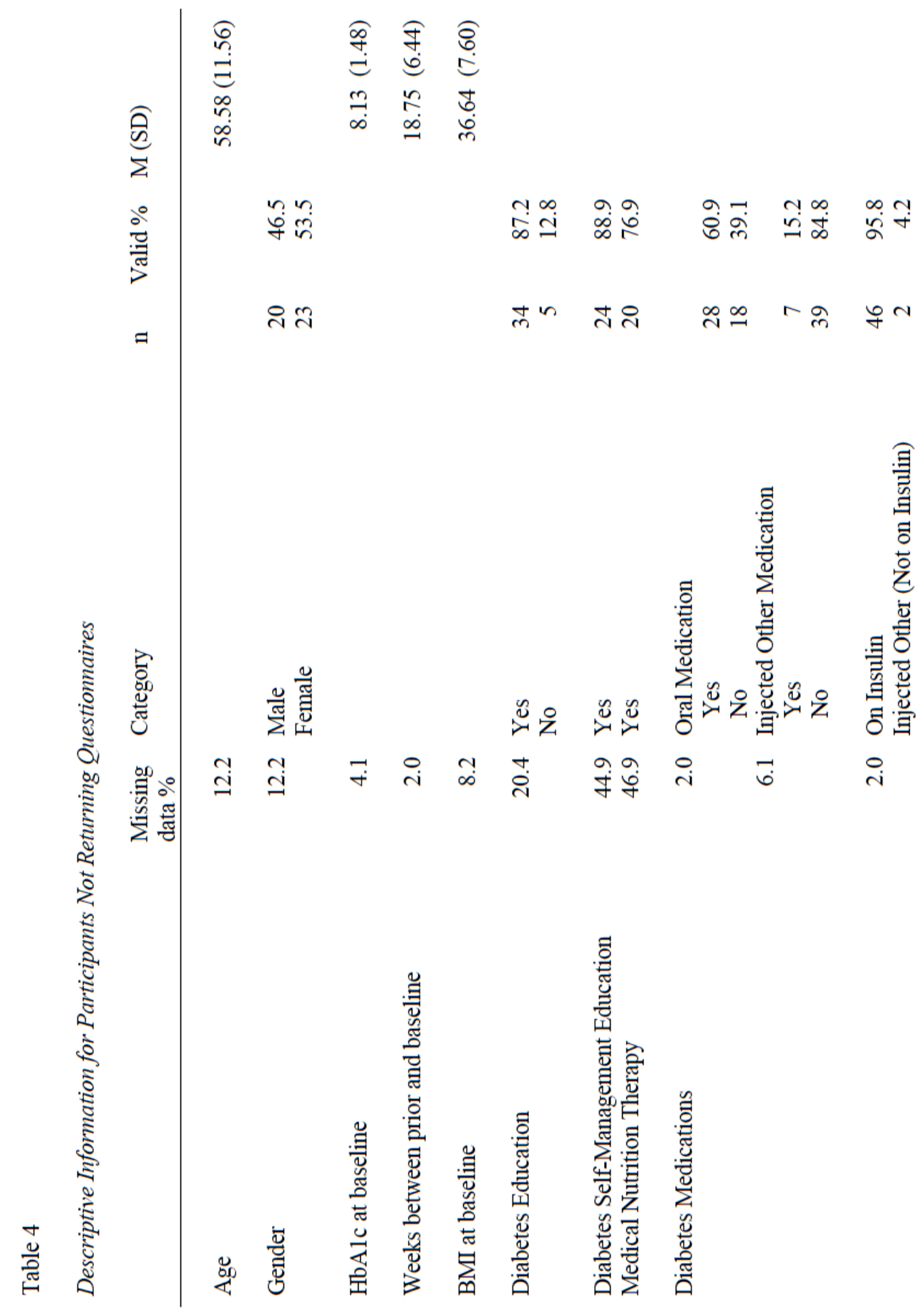




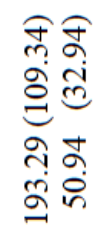

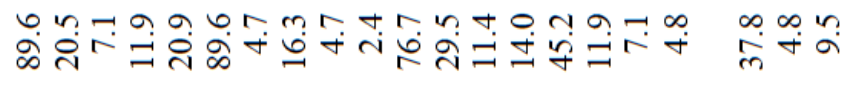

$$
\begin{aligned}
& \text { กุตn }
\end{aligned}
$$

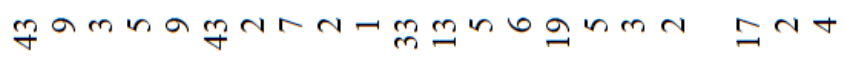

$$
\begin{aligned}
& \underline{n-1}-n
\end{aligned}
$$

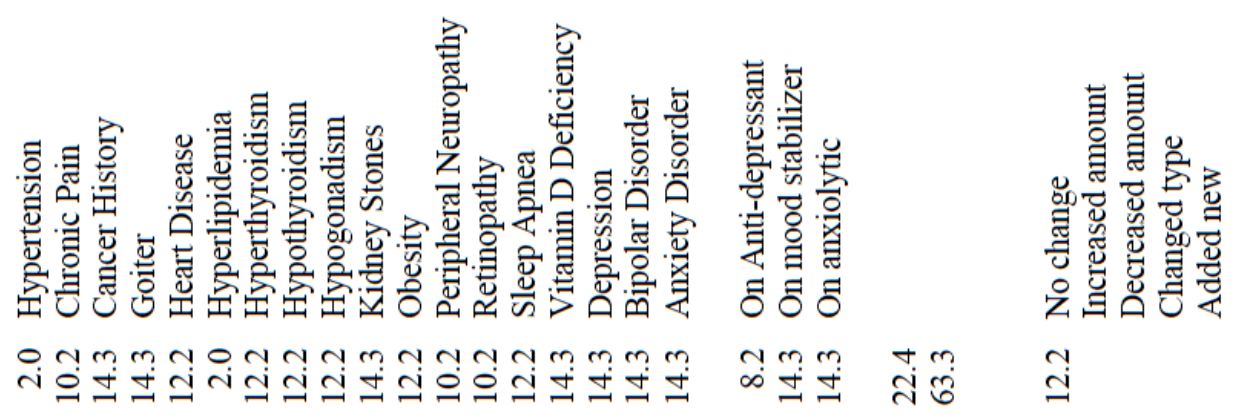

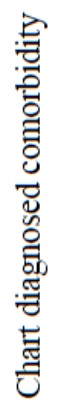
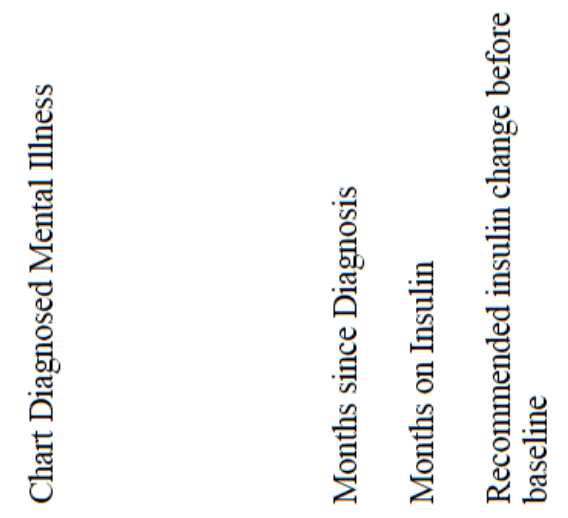


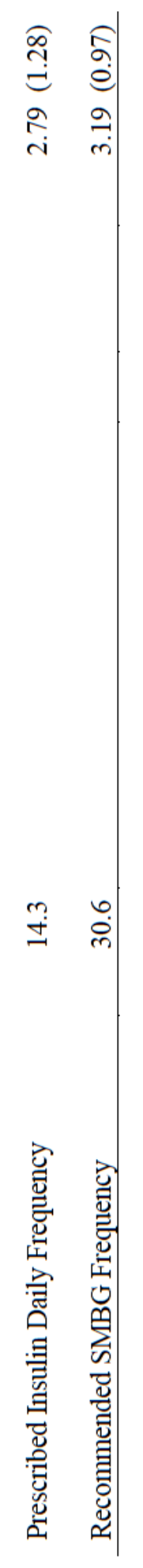




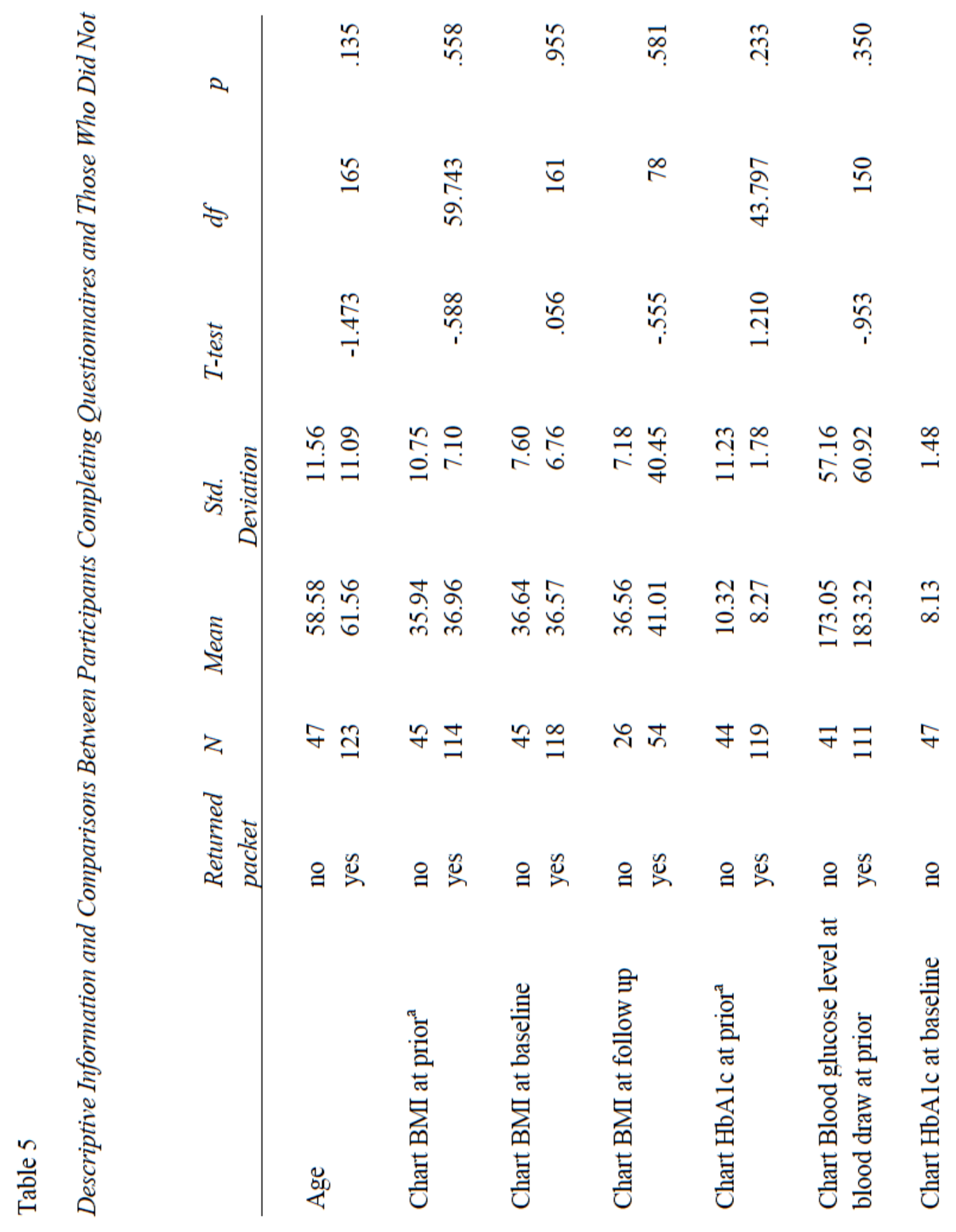




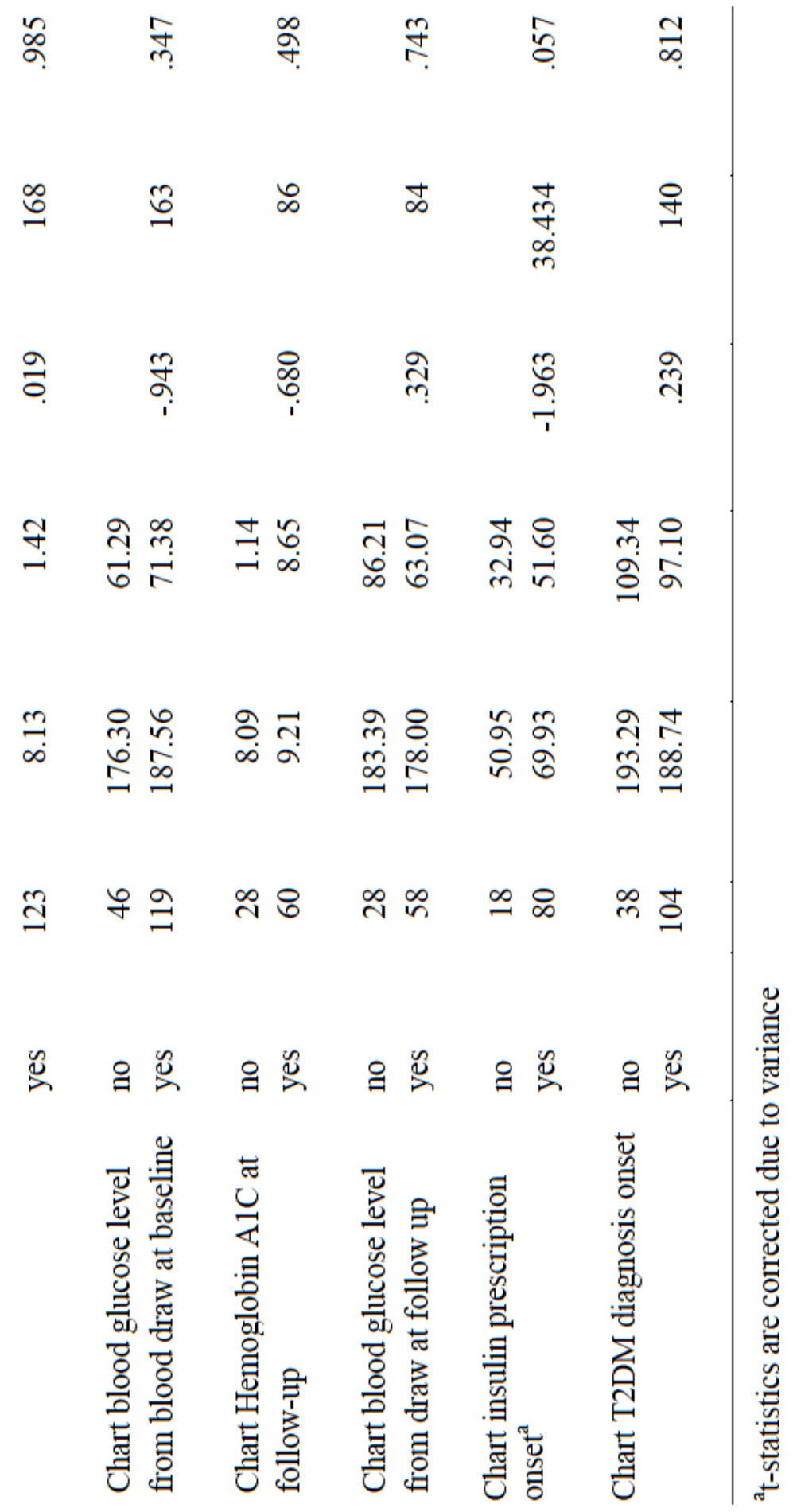




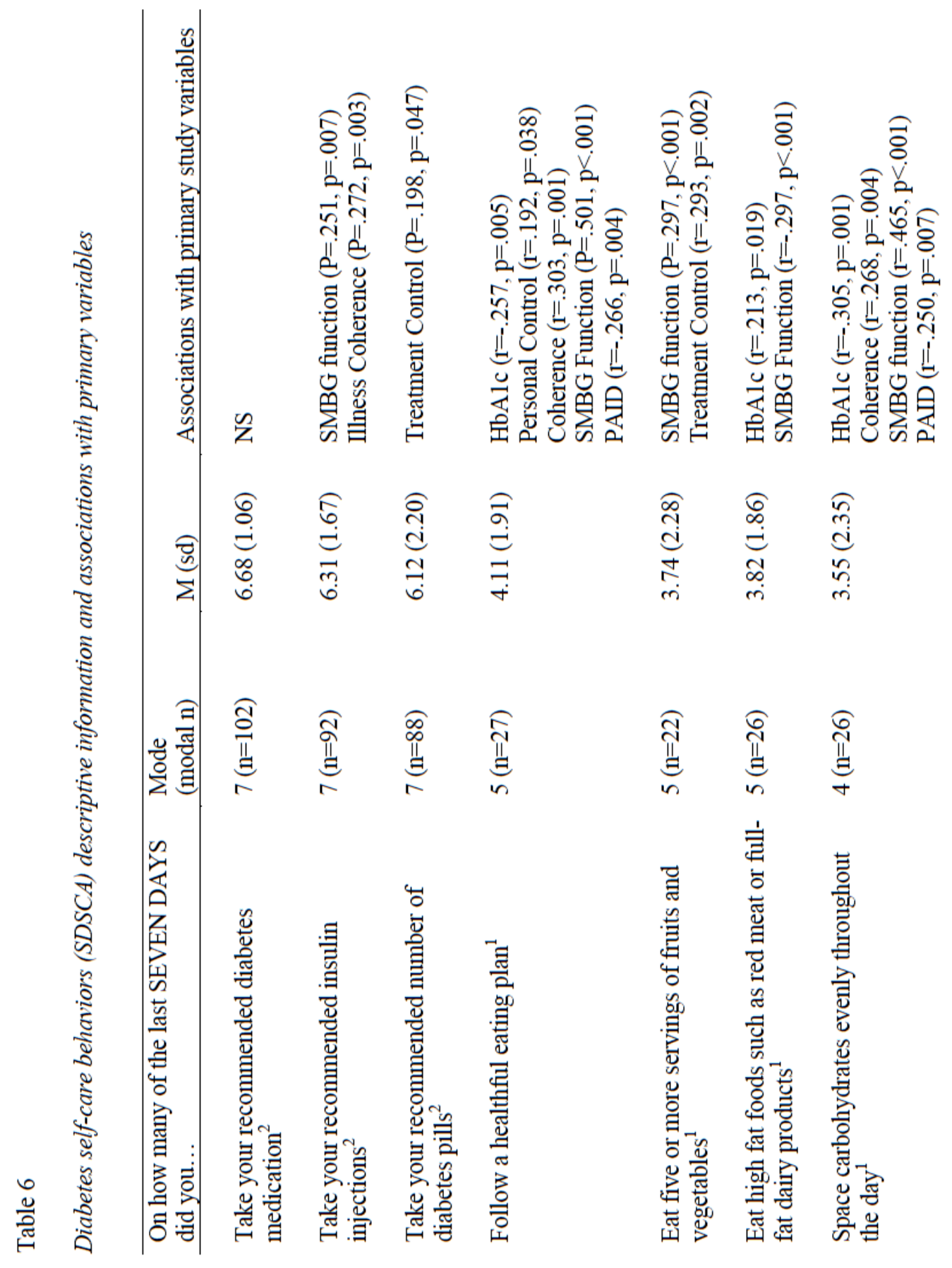




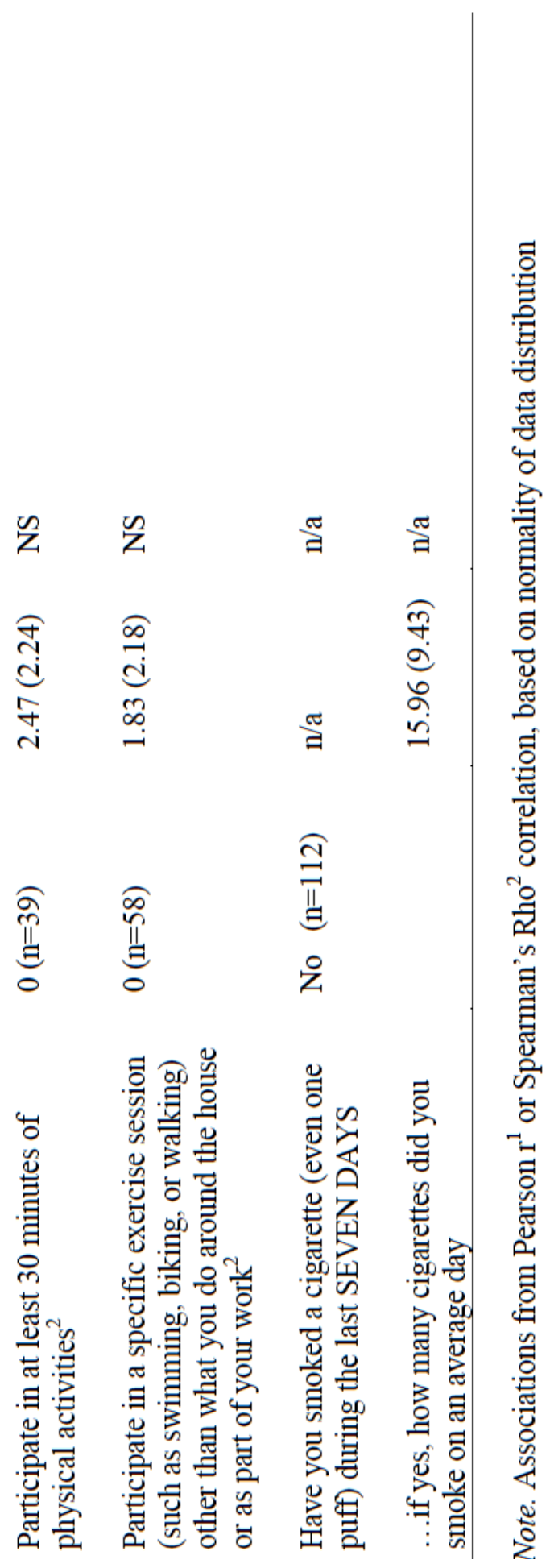




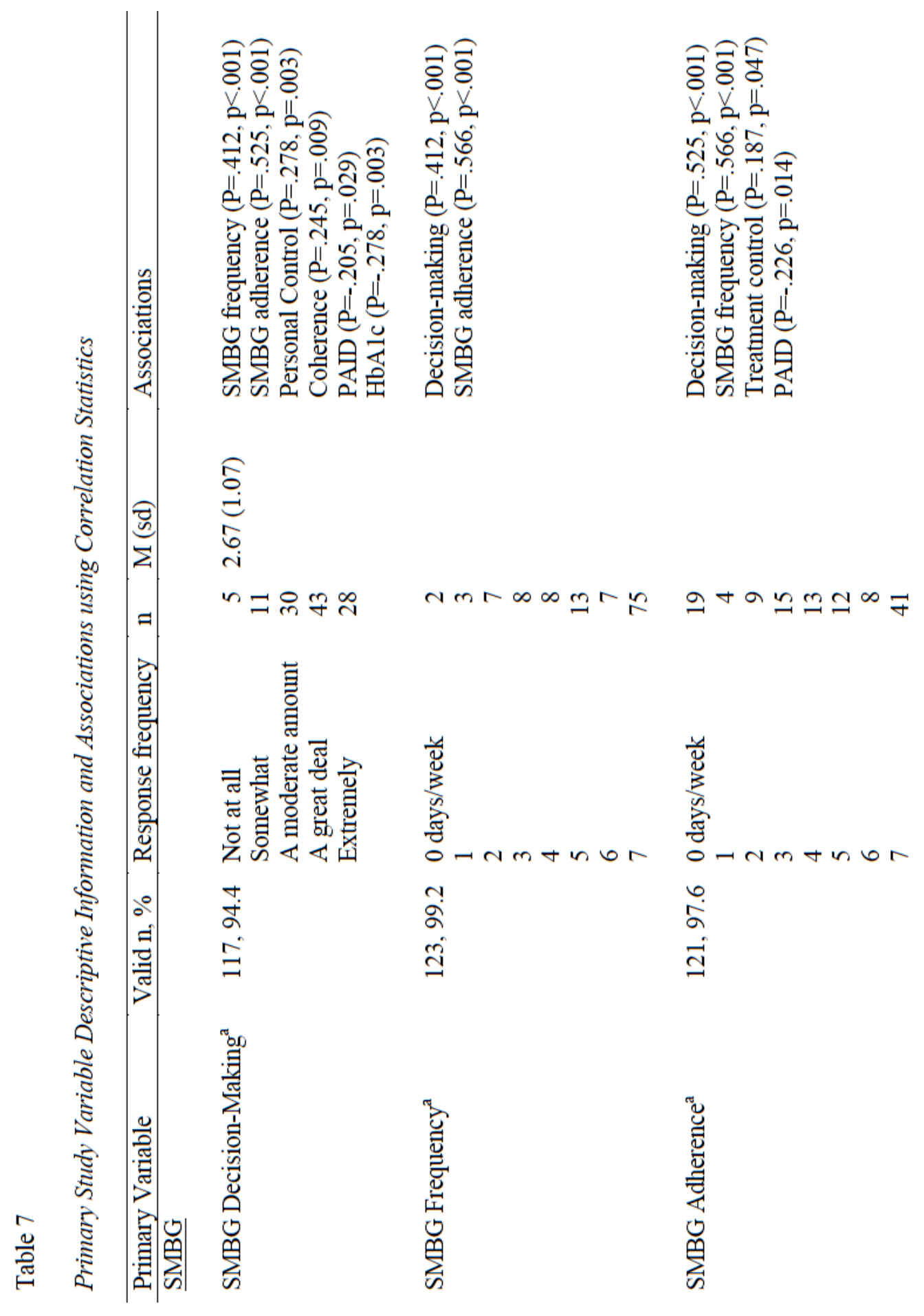




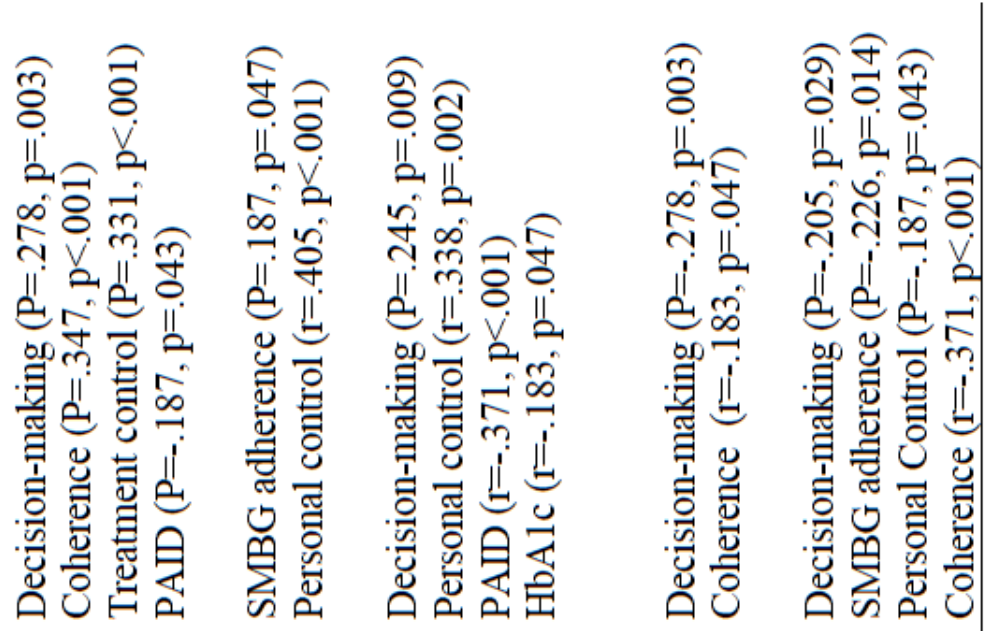

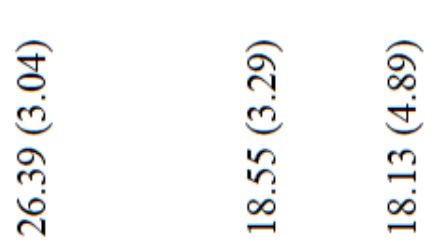

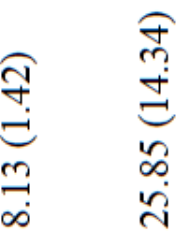

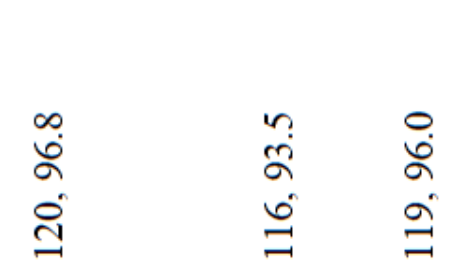

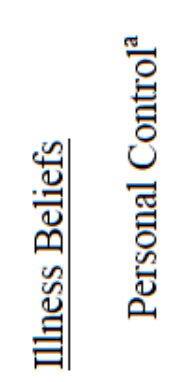

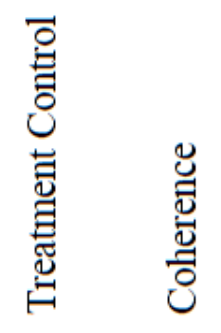

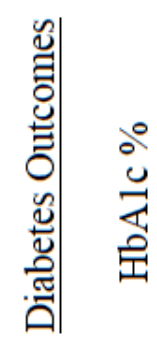

$$
\begin{aligned}
& \text { }
\end{aligned}
$$




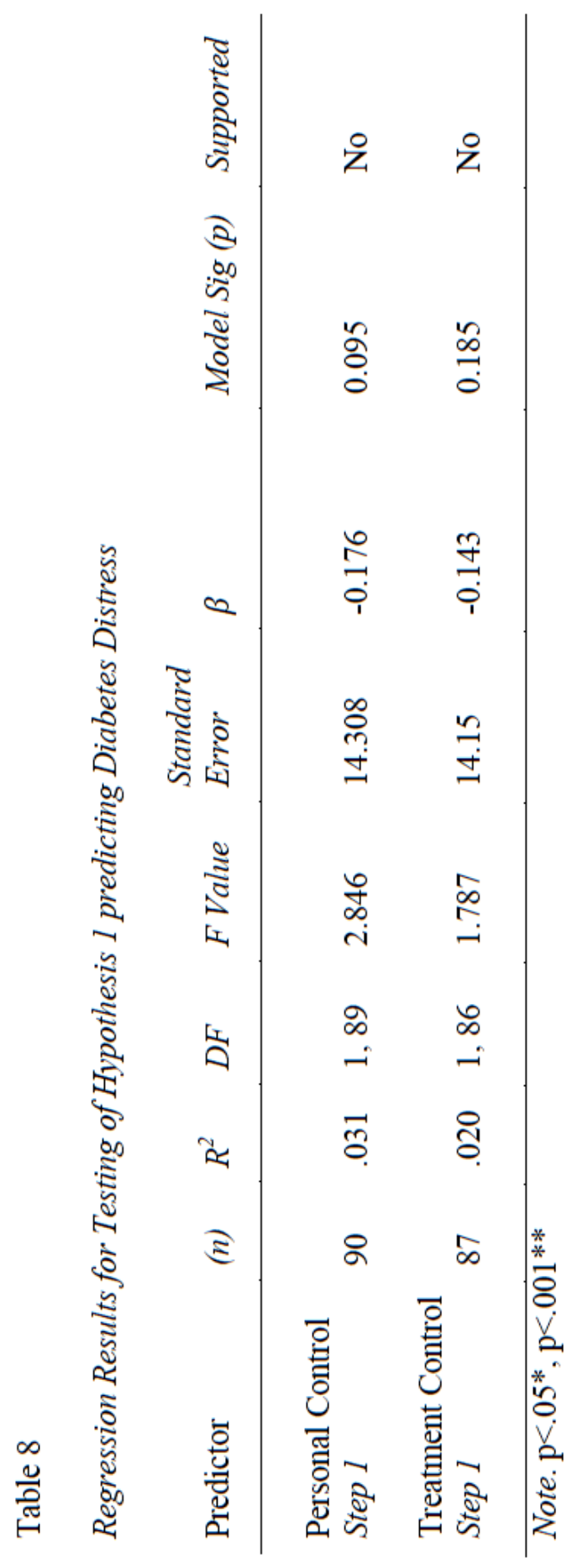




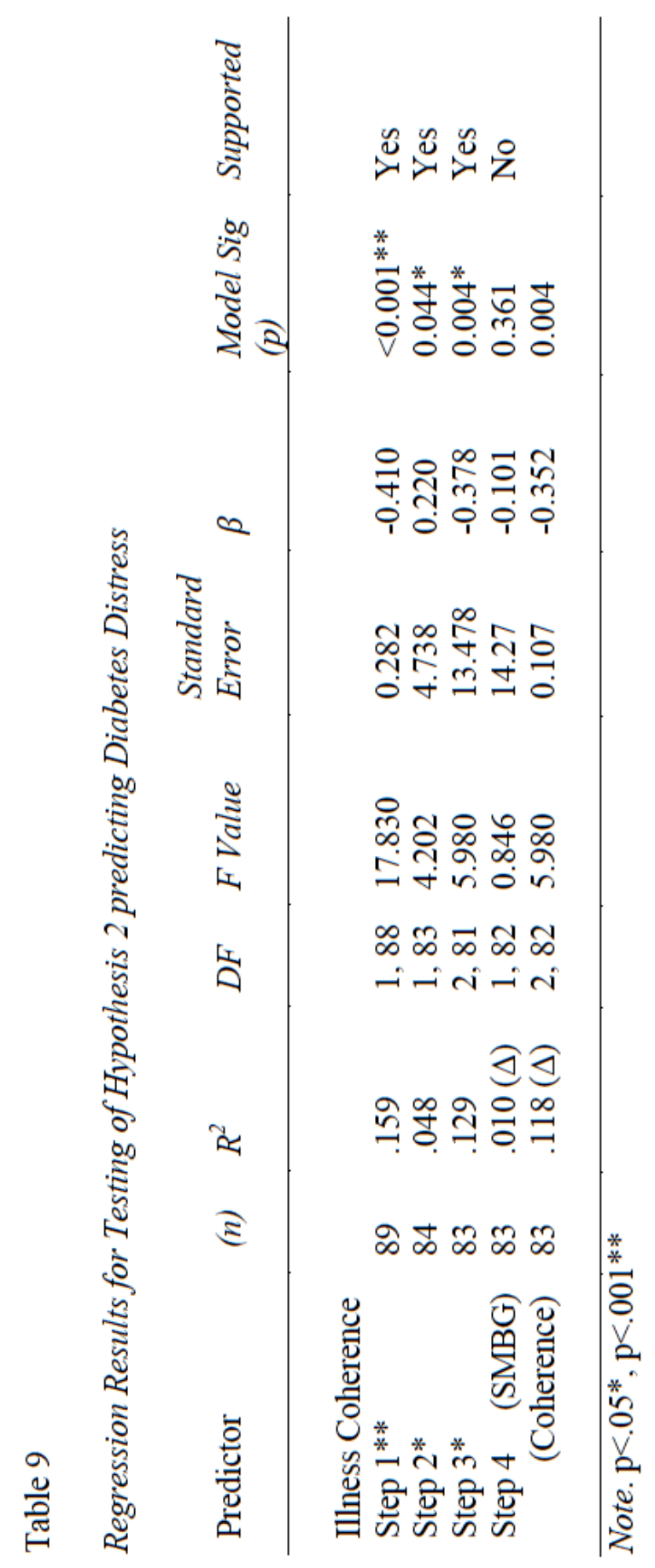




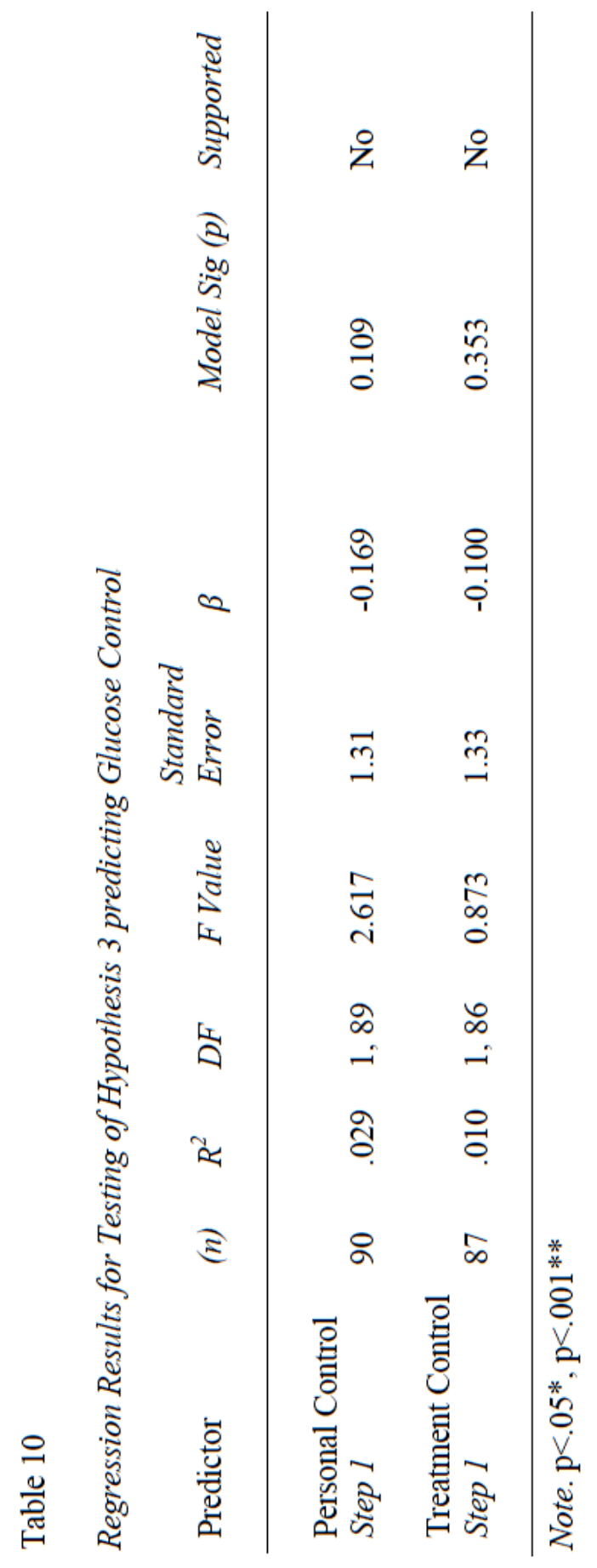




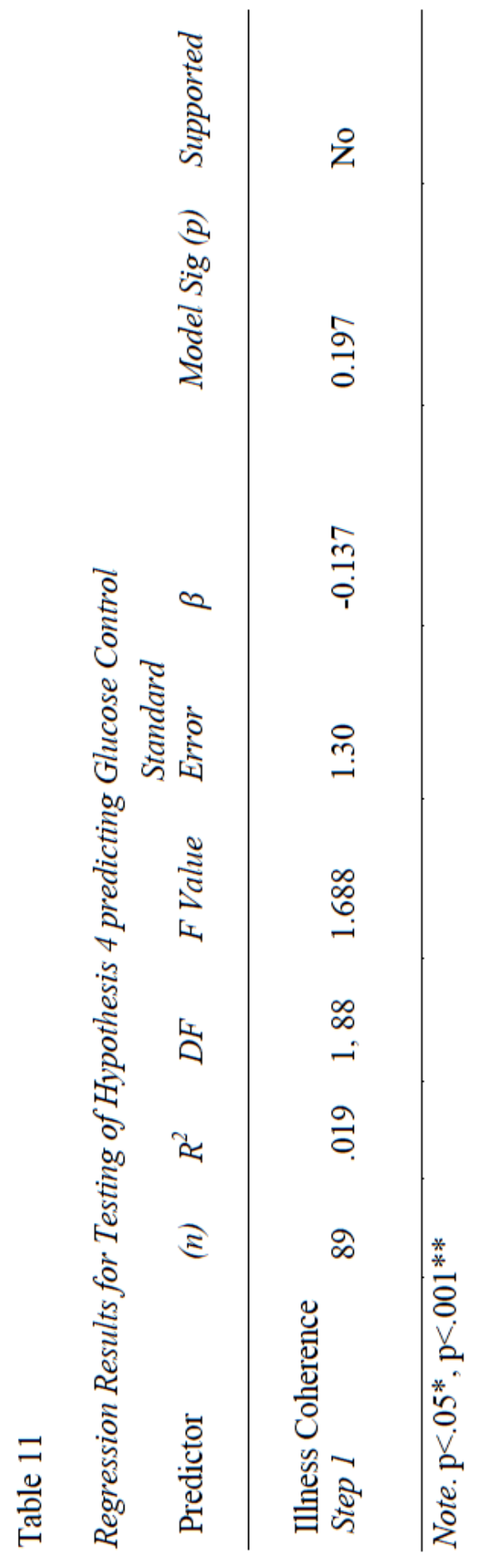




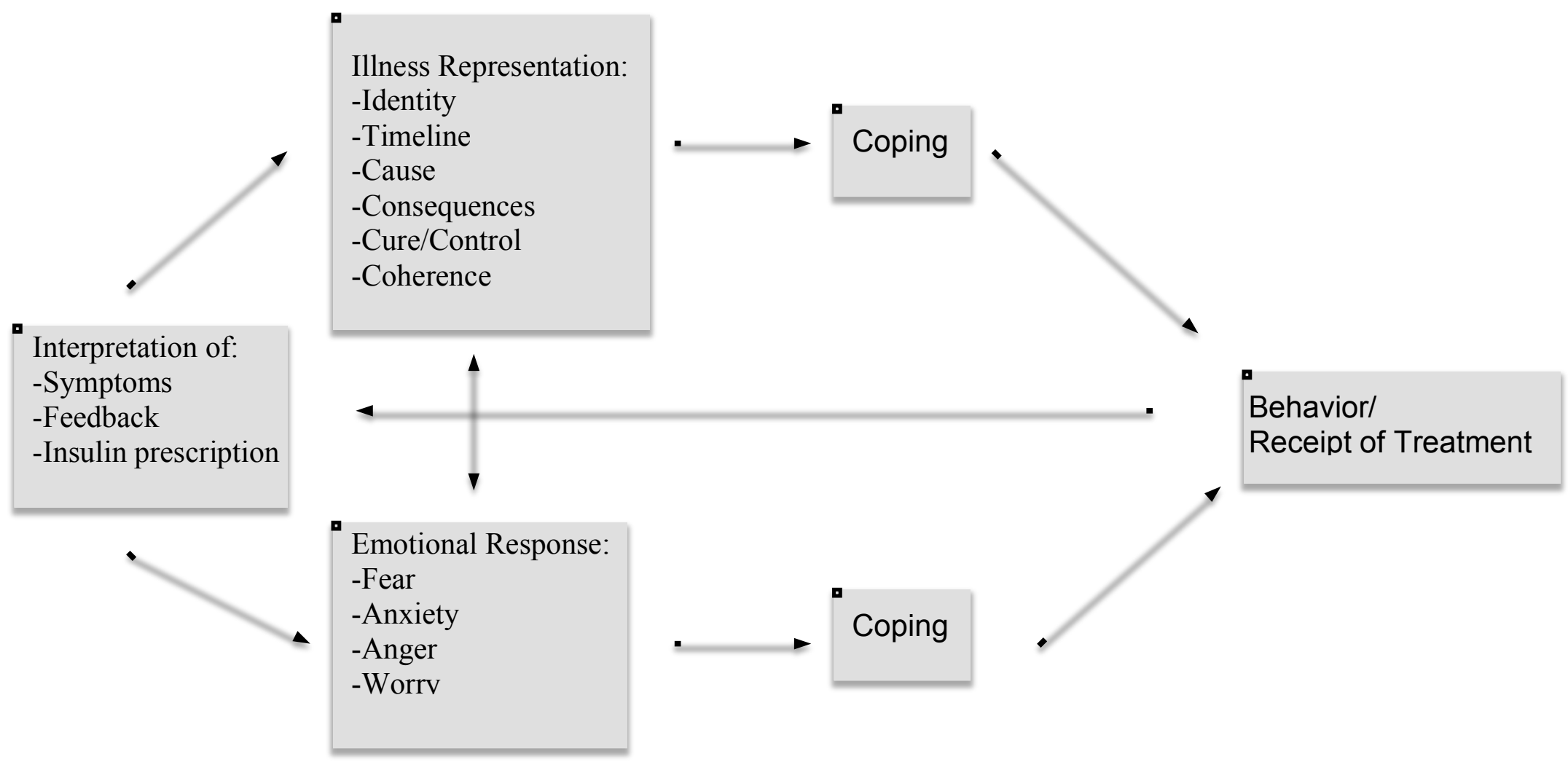

Figure 1. The Self-Regulation Model and Illness Behavior (adapted from Elwy et al, 2011). This figure represents to basic model of self-regulatory health behaviors. 


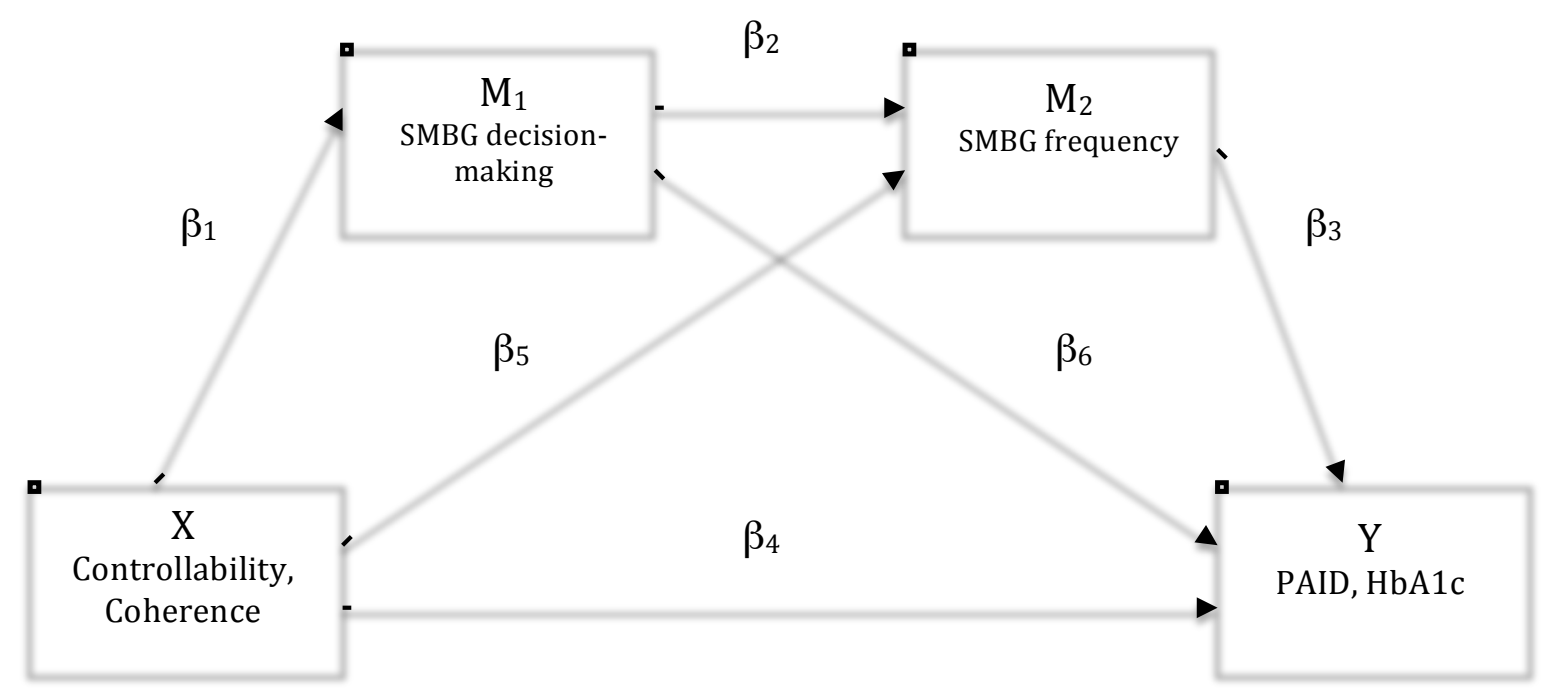

Figure 2. Path diagram of the three-path mediated effect model (adapted from Taylor et al., 2008). 


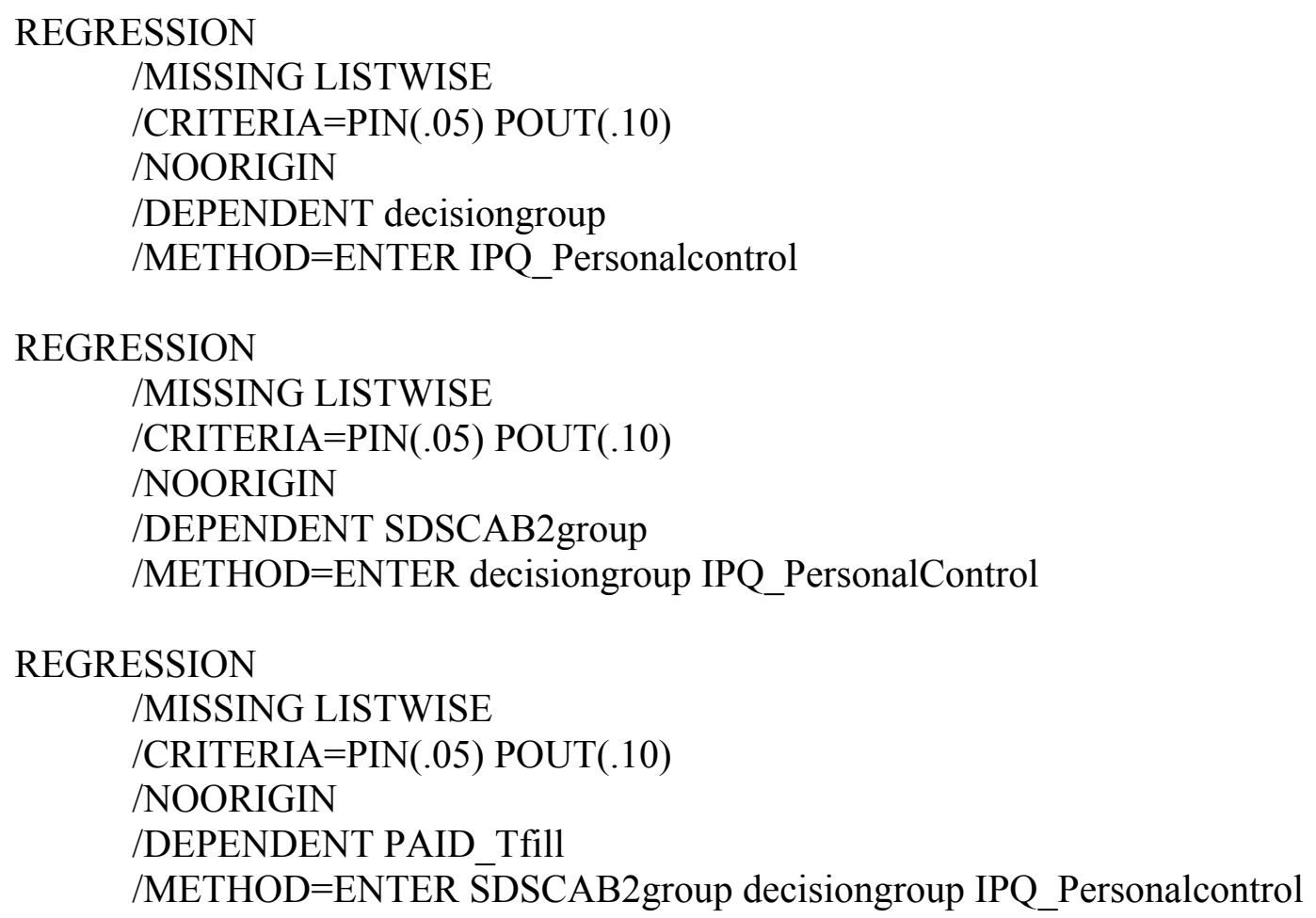

\section{REGRESSION}

/MISSING LISTWISE

/CRITERIA=PIN(.05) POUT(.10)

/NOORIGIN

/DEPENDENT SDSCAB2group

$/$ METHOD=ENTER decisiongroup IPQ_PersonalControl

\section{REGRESSION}

/MISSING LISTWISE

$/$ CRITERIA=PIN(.05) POUT(.10)

/NOORIGIN

/DEPENDENT PAID Tfill

/METHOD=ENTER SDSCAB2group decisiongroup IPQ_Personalcontrol

\section{Model Summary}

\begin{tabular}{|c|c|c|c|c|}
\hline Model & $\mathrm{R}$ & $\mathrm{R}$ Square & $\begin{array}{c}\text { Adjusted R } \\
\text { Square }\end{array}$ & $\begin{array}{c}\text { Std. Error of } \\
\text { the Estimate }\end{array}$ \\
\hline 1 & & & & $\varepsilon_{1}$ \\
\hline
\end{tabular}

a. Predictors: (Constant), IPQ-R control subscale

b. Dependent Variable: SMBG decision-making

\begin{tabular}{|c|c|c|c|c|c|}
\hline & \multicolumn{2}{|c|}{ Unstandardized Coefficients } & $\begin{array}{l}\text { Standardized } \\
\text { Coefficients }\end{array}$ & & \\
\hline Model & $\mathrm{B}$ & Std. Error & Beta & $\mathrm{t}$ & Sig. \\
\hline $\begin{array}{l}1 \quad \text { (constant) } \\
\text { IPQ } \\
\text { (control) }\end{array}$ & $\mathrm{b}_{1}$ & $\mathrm{~S}_{\mathrm{b} 1}$ & $\begin{array}{l}\beta_{01} \\
\beta_{1}\end{array}$ & & \\
\hline
\end{tabular}

a. Dependent Variable: SMBG decision-making

Model Summary

\begin{tabular}{|c|c|c|c|c|}
\hline Model & $\mathrm{R}$ & $\mathrm{R}$ Square & $\begin{array}{c}\text { Adjusted R } \\
\text { Square }\end{array}$ & $\begin{array}{c}\text { Std. Error of } \\
\text { the Estimate }\end{array}$ \\
\hline 1 & & & & $\varepsilon_{2}$ \\
\hline
\end{tabular}

a. Predictors: (Constant), SMBG decision-making, IPQ-R control subscale

b. Dependent Variable: SMBG frequency (SDSCA) 


\begin{tabular}{|c|c|c|c|c|c|}
\hline & \multicolumn{2}{|c|}{ Unstandardized Coefficients } & $\begin{array}{l}\text { Standardized } \\
\text { Coefficients }\end{array}$ & & \\
\hline Model & $\mathrm{B}$ & Std. Error & Beta & $\mathrm{t}$ & Sig. \\
\hline $\begin{array}{ll}1 & \text { (constant) } \\
& \text { SMBGdm } \\
& \text { IPQ } \\
\text { (control) }\end{array}$ & $\begin{array}{l}b_{2} \\
b_{3}\end{array}$ & $\begin{array}{l}\mathrm{s}_{\mathrm{b} 2} \\
\mathrm{~s}_{\mathrm{b} 3}\end{array}$ & $\begin{array}{l}\beta_{02} \\
\beta_{2} \\
\beta_{3}\end{array}$ & & \\
\hline
\end{tabular}

a. Dependent Variable: SMBG frequency (SDSCA)

Model Summary

\begin{tabular}{|c|c|c|c|c|}
\hline Model & $\mathrm{R}$ & R Square & $\begin{array}{c}\text { Adjusted R } \\
\text { Square }\end{array}$ & $\begin{array}{c}\text { Std. Error of } \\
\text { the Estimate }\end{array}$ \\
\hline 1 & & & & $\varepsilon_{3}$ \\
\hline
\end{tabular}

a. Predictors: (Constant), SMBG frequency (SDSCA), SMBG decision-making, IPQ-R control subscale

b. Dependent Variable: PAID total score

\begin{tabular}{|c|c|c|c|c|c|}
\hline & Unstandardized & efficients & $\begin{array}{l}\text { Standardized } \\
\text { Coefficients }\end{array}$ & & \\
\hline Model & $\mathrm{B}$ & Std. Error & Beta & $\mathrm{t}$ & Sig. \\
\hline $\begin{array}{ll}1 & \text { (constant) } \\
& \text { SMBGf } \\
& \text { SMBGdm } \\
& \text { IPQ } \\
\text { (control) }\end{array}$ & & & $\begin{array}{l}\beta_{03} \\
\beta_{4} \\
\beta_{5} \\
\beta_{6}\end{array}$ & & \\
\hline
\end{tabular}

a. Dependent Variable: PAID total score

Figure 3. Standard SPSS output and hypothesis testing, example based on H1. 


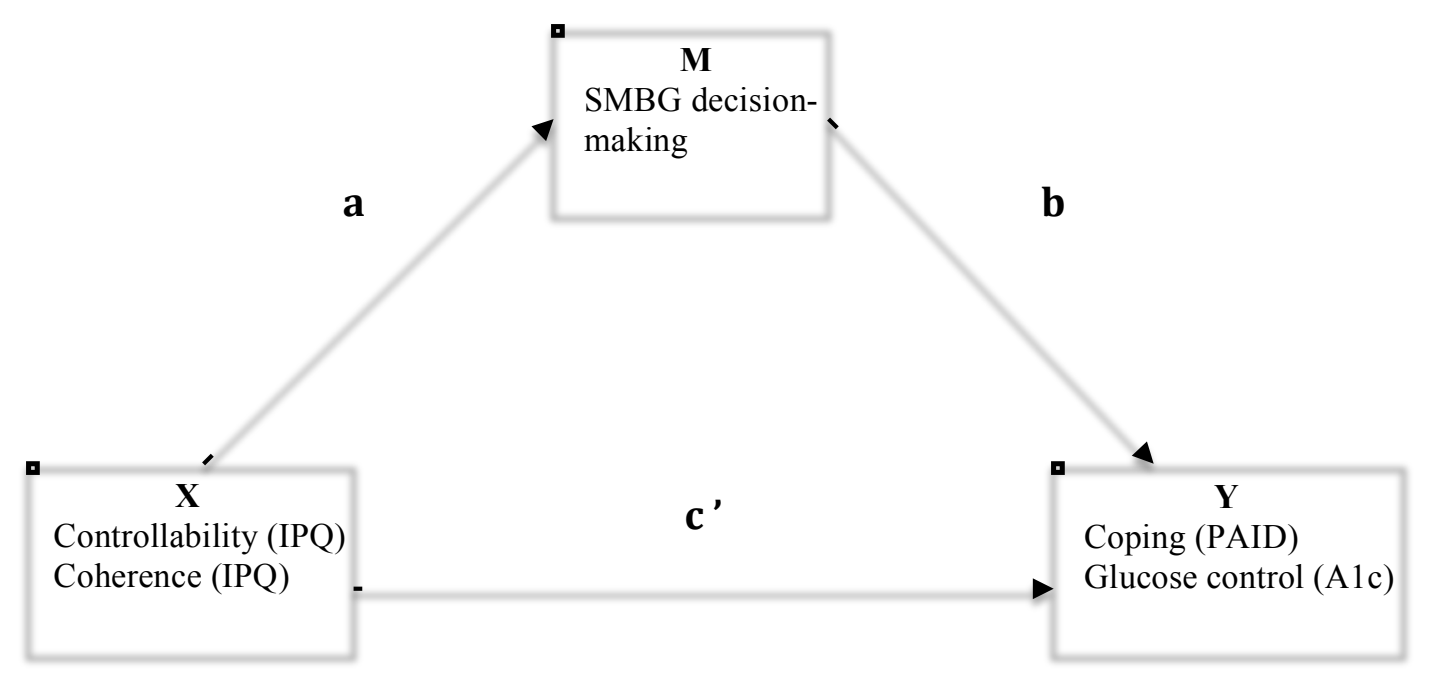

Figure 4. Path diagram of revised simple mediation model (Baron \& Kenny, 1986).

The effect of $X$ on $Y$ is hypothesized to have an indirect effect on $M$ (c' represents the direct effect). 


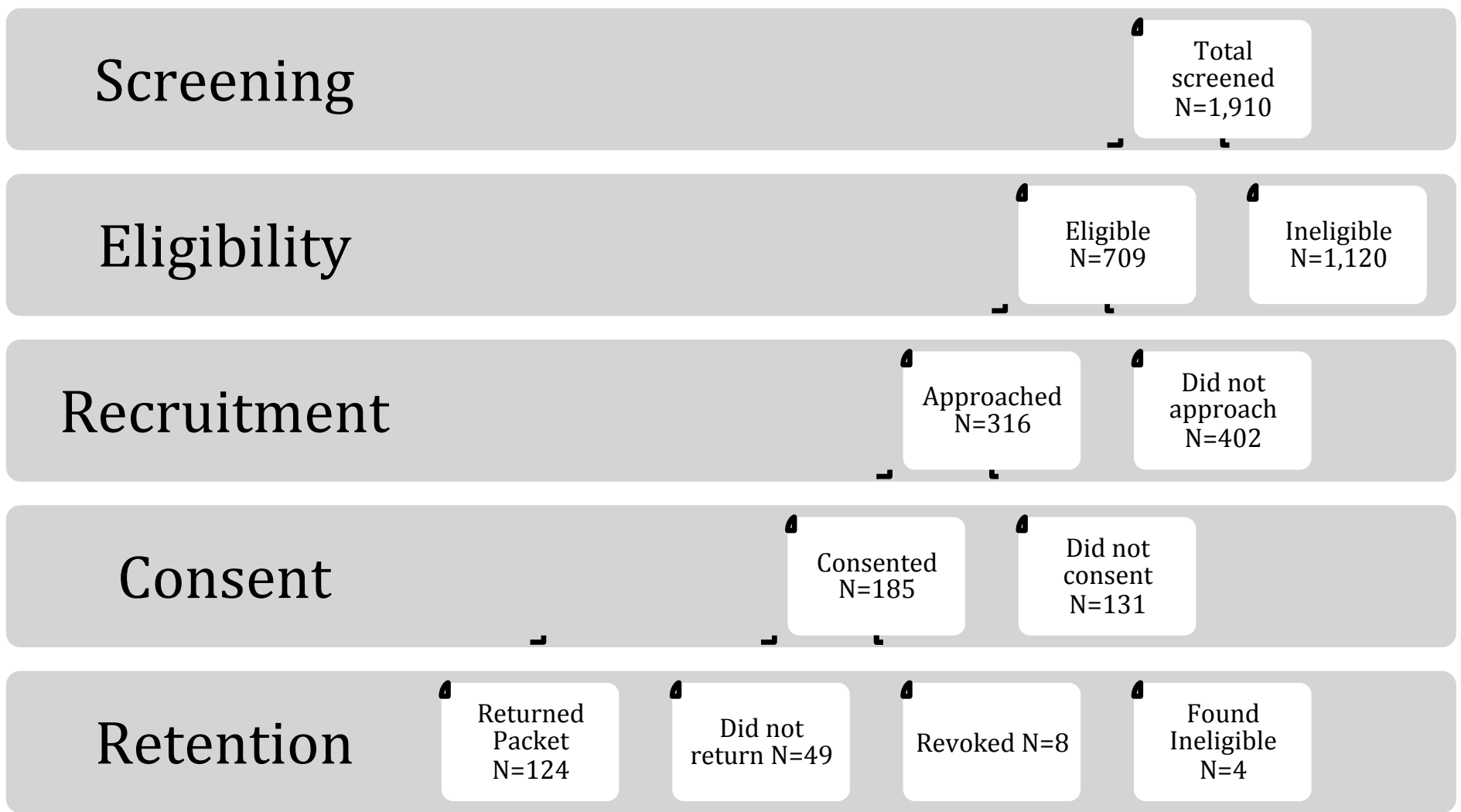

Figure 5. Participant flow diagram of recruitment and retention numbers. 


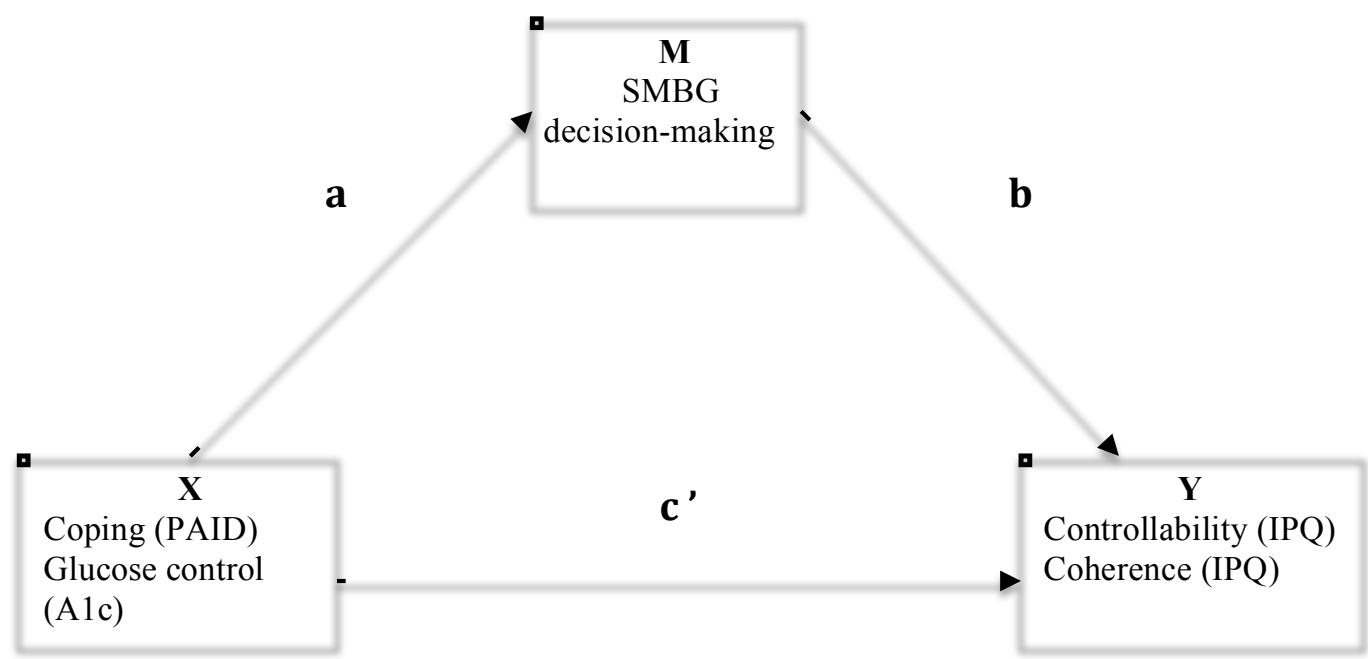

Figure 6. Path diagram of revised simple mediation model in reverse order (Baron \& Kenny, 1986). The effect of $X$ on $Y$ is hypothesized to have an indirect effect on $M$ (c' represents the direct effect). 


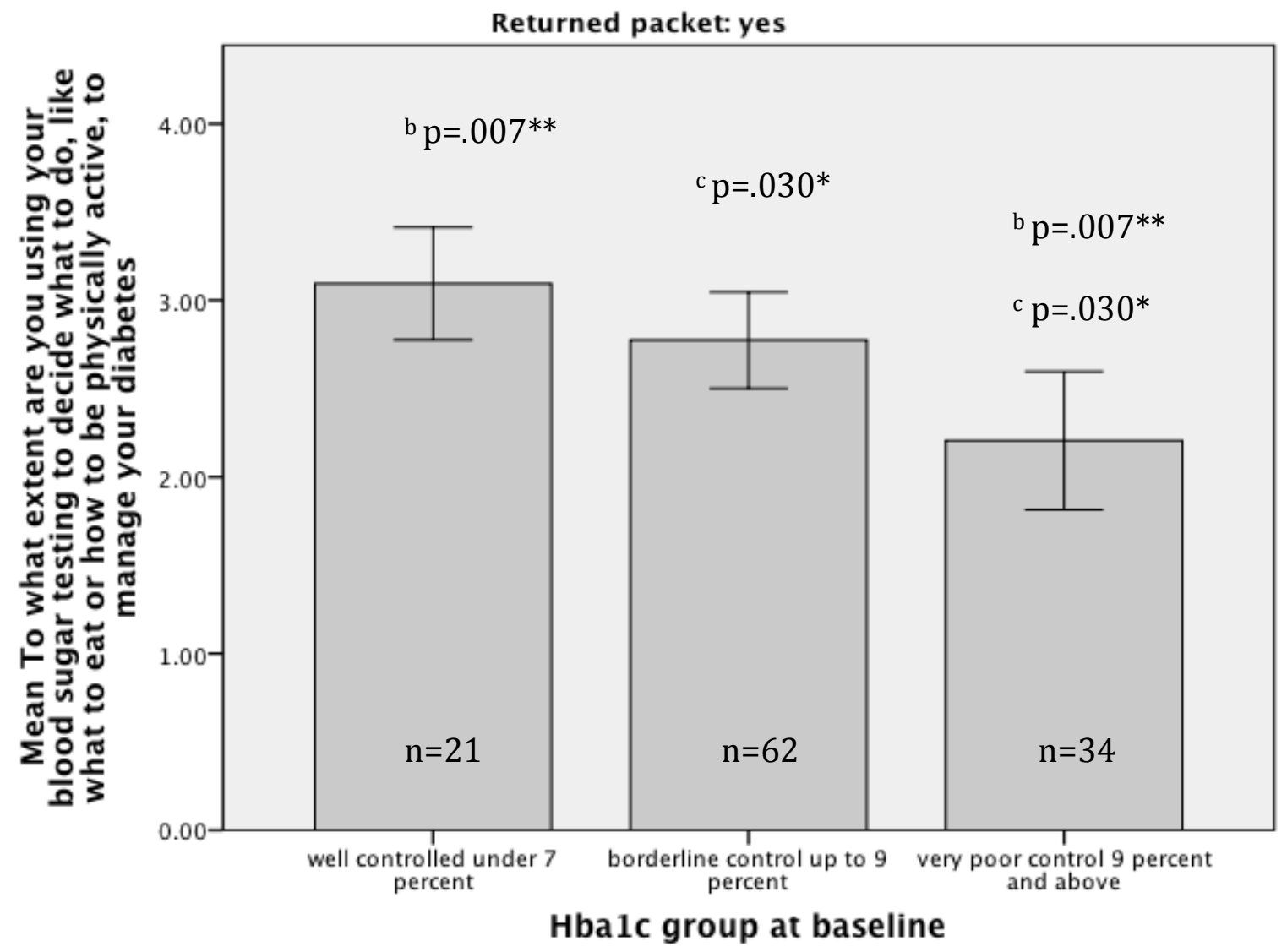

Figure 7. Mean SMBG decision-making use by glucose control group.

a $p$-values are from Tukey HSD post hoc analyses comparing means ranks for response on SMBG decision-making item, $\mathrm{H}(2)=9.796, \mathrm{p}=.007$

b difference between "well controlled" and "very poor control" group $(\mathrm{p}=.007)$

c difference between "borderline" group and "very poor control" $(p=.030)$ 


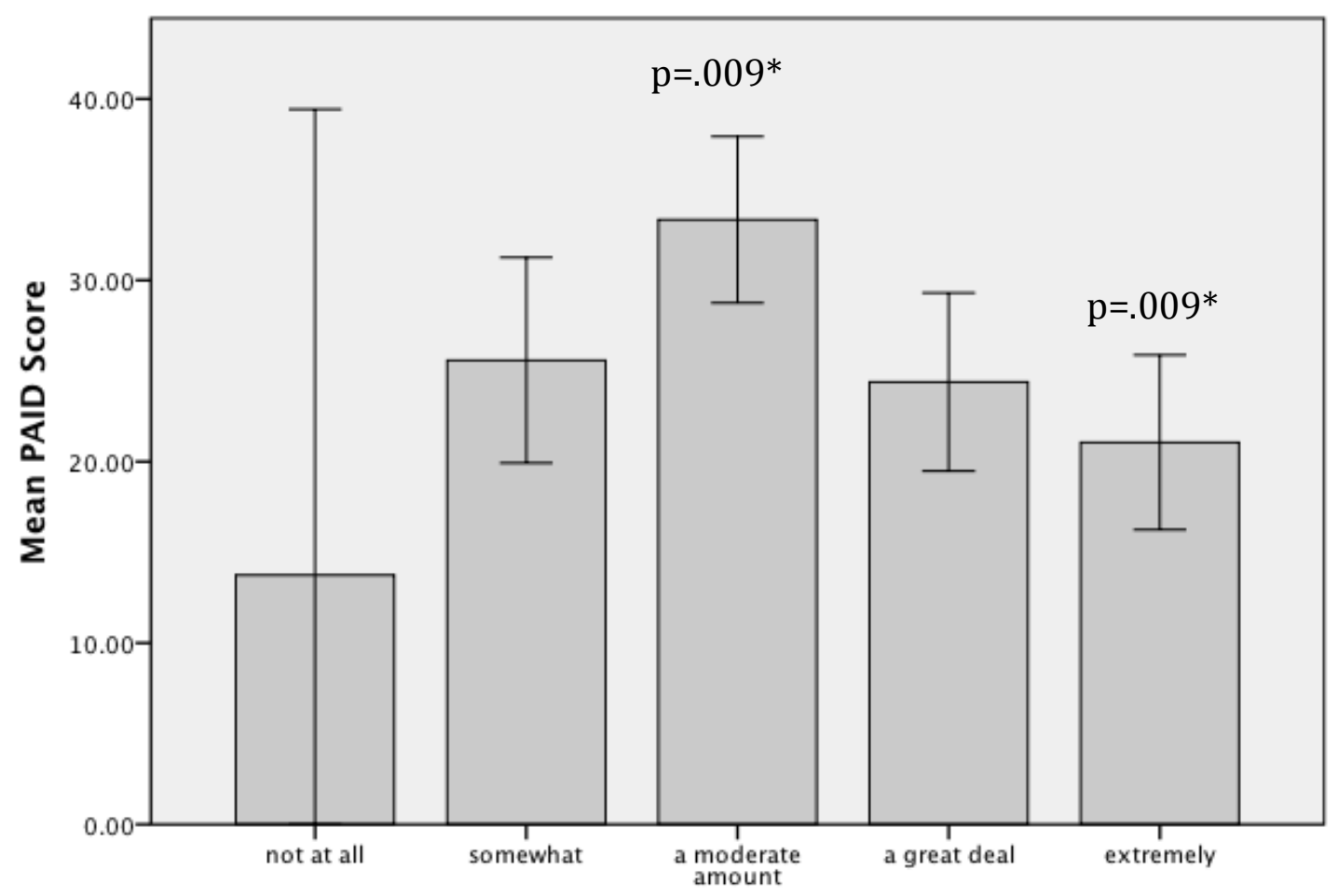

To what extent are you using your blood sugar testing to decide what to do, like what to eat or how to be physically active, to manage your diabetes

Figure 8. Mean diabetes distress score by self-monitoring of blood glucose decision-making group. P-value indicates a significant difference in post hoc testing (Tukey HSD). 


\section{REFERENCES}

Aalto, A. \& Uutela, A. (1997). Glycemic control, self-care behaviors, and psychosocial factors among insulin treated diabetics: a test of an extended health belief model. International Journal of Behavioral Meidcine, 4 (3), 191-214.

Aarts, H. (2007). Health and goal-directed behavior: The nonconscious regulation and motivation of goals and their pursuit. Health Psychology Review, 1(1), 53-82.

American Diabetes Association. (2012). Position Statement: Standards of medical care in diabetes - 2012. Diabetes Care, 35(Supplement 1), s11-s63.

American Diabetes Association (2013). Position Statement: Standards of medical care in diabetes - 2013. Diabetes Care, 36 (Supplement 1), s11-s66.

Bairey Merz, C. N., Buse, J. B., Tuncer, D., \& Twillman, G. B. (2002). Physician attitudes and practices and patient awareness of the cardiovascular complications of diabetes. Journal of the American College of Cardiology, 40(10).

Bandura, A. (1977). Self-efficacy: Toward a unifying theory of behavioral change. Psychological Review, 84, 191-215.

Baron, R. M., \& Kenny, D. A. (1986). The moderator-mediator variable distinction in social psychological research: Conceptual, strategic and statistical considerations. Journal of Personality and Social Psychology, 51, 1173-1182.

Beck A., Steer R. (1987). BDI, Beck depression inventory : manual. Psychological Corp: New York, Harcourt Brace Jovanovich: San Antonio, TX. 
Berlin, I., Sachon, C., \& Grimaldi, A. (2005). Identification of factors associated with impaired hypoglycaemia awareness in patients with type 1 and type 2 diabetes mellitus. Diabetes Metab, 31, 246-251.

Beverly, E. A. (2014). Stressing the Importance of Diabetes Distress: a Comment on Baek et al. Annals of Behavioral Medicine, 48, 137 - 139.

Brega, A., Ang., A., Vega, W., Jiang, L., Beals, J., et al. (2012). Mechanisms underlying the relationship between health literacy and glycemic control in American Indians and Alaska Natives. Patient Education and Counseling, 88, 61-68.

Broadbent, E., Petrie, K. J., Main, J., \& Weinman, J. A. (2006). The brief illness perception questionnaire. Journal of Psychosomatic Research, 60, 631-637.

Brown, J. B., Harris, S. B., Webster-Bogaert, S., Wetmore, S., Faulds, C., \& Stewart, M. (2002). The role of patient, physician and systemic factors in the management of type 2 diabetes. Family Practice, 19, 344-349.

Cameron, L. D., \& Leventhal, H. (Eds.). (2003). the self-regulation of health and illness behavior. New York, NY: Routledge.

Centers for Disease Control (2011). National Diabetes fact sheet: national estimates and general information on diabetes and prediabetes in the United States, 2011 Retrieved March 25, 2012.

Ceriello, A. (2012). Editorial: Self-monitoring of blood glucose in type 2 diabetes: Is the debate (finally) ending? Diabetes Research and Clinical Practice, 97, 1-2.

Clar, C., Barnard, E., Cummins, E., Royle, P., Waugh, N. (2010). Self-monitoring of blood glucose in type 2 diabetes: systematic review. Health Technology Assessment. 14(12). 
Cohen, J. (1992). Statistical Power Analysis. Current Directions in Psychological Science, 1(3): 98-101.

Cox, D., Gonder-Frederick, L., Ritterband, L., et al. (2006). Blood glucose awareness training: what is it, where is it, and where is it going? Diabetes Spectrum, 9(1), 43-49.

Davies, M., Lavalle-Gonzalez, Storms, F., \& Gomis, R. (2008). Initiation of insulin glargine therapy in type 2 diabetes subjects suboptimally controlled on oral antidiabetic agents: results from the AT.LANTUS trial. Diabetes, Obesity and Metabolism, 10, 387-399.

Deakin, TA, McShane, CE, Cade, JE, Williams, R. (2009). Group based training for selfmanagement strategies in people with type 2 diabetes mellitus (Review). The Cochrane Collaboration. Issue 1.

Deakin, T.A., Cade, J.E., Williams, R., Greenwood, D.C. (2006). Structured patient education: the Diabetes X-PERT Programme makes a difference. Diabetic Medicine, 23: 944-954.

De Groot, M., Anderson, R., Freedland, K., Clouse, R. E., \& Lustman, P. J. (2001). Association of depression and diabetes complications: A Meta-analysis. Psychosomatic Medicine, 63(4), 619-630.

Diabetes Research Group (1990). Diabetes control and complications trial (DCCT): update. Diabetes Care, 13(4), 427-433.

Diefenbach, M. A., \& Leventhal, H. (1996). The Common-Sense model of illness representation: Theoretical and Practical Considerations. Journal of Social Distress and the Homeless, 5(1), 11-38. 
Elwy, A. R., Yeh, J., Worcester, J., \& Eisen, S. V. (2011). An Illness Perception Model of Primary Care Patients' Help Seeking for Depression Qualitative Health Research, 21(11), 1495-1507.

Faul, F., Erdfelder, E., Buchner, A., Lang, A-G. (2009). Statistical power analyses using G*Power 3.1: Tests for correlation and regression analyses. Behavior Research Methods, 41 (4), 1149-1160.

Fisher, L., Polonsky, W., Parkin, C., Jelsovkt, Z., Petersen, B., Wagner, R. (2012). The impact of structured blood glucose testing on attitudes toward self-management among poorly controlled, insulin-naïve patients with type 2 diabetes. Diabetes Research and Clinical Practice, 96, 149-155.

Fu, A. Z., Qiu, Y., \& Radican, L. (2009). Impact of fear of insulin or fear of injection on treatment of outcomes of patients with diabetes. Current Medical Research \& Opinion, 25(6), 1413-1420.

Gilman, S. L. (2011). Representing Health and Illness: Thoughts for the 21 st century. $J$ Med Humanit, 32, 69-75.

Glantz, K. (2012). Theory at a Glance: A Guide for Health Promotion Practice Retrieved Mar 20, 2012, from http://www.cancer.gov/PDF/481f5d53-63df-41bc-bfaf5aa48ee1da4d/TAAG3.pdf

Griva, K., Myers, L., Newman, S. (2000). Illness perceptions and self efficacy beliefs in adolescents and young adults with insulin dependent diabetes mellitus. Psychology and Health, 15. 733-750.

Gomersall, T., Madill, A., Summers, L. (2011). A metasynthesis of the self-management of type 2 diabetes. Qualitative Health Research, 21, 853-71. 
Gonder-Frederick, L., Cox, D. J., Bobbitt, S. A., \& Pennebaker, J. W. (1989). Mood changes associated with blood glucose fluctuations in insulin-dependent diabetes mellitus. Health Psychology, 8(1), 45-49.

Gonzalez, J., Delahanty, L. Safren, S., Meigs, J., Grant, R. (2008a). Differentiating symptoms of depression from diabetes-specific distress: relationships with selfcare in type 2 diabetes. Diabetologia 51: 1822-1825.

Gonzalez, J. S., Serpa, L., Peyrot, M., Mimiaga, M., McCarl, L., Safren, S., \& Collins, E. M. (2008b). Depression and diabetes treatment nonadherence: A meta-analysis. Diabetes Care, 31, 2398-2403.

Gonzalez, J., Safren, S., Cagliero, E., Wexler, D., Delehanty, L., et al. (2007). Depression, Self-care, and medication adherence in type 2 diabetes: relationships across the full range of symptom severity. Diabetes Care. 30; 2222-2227.

Grzywacz, J. G., Arcury, T. A., Ip, E. H., Chapman, C., Kirk, J. K., Bell, R. A., \& Quandt, S. A. (2011). Older adults' common sense model of diabetes. American Journal of Health Behavior, 35(3), 318-333.

Hagger, M. S. (2010). Self-regulation: an important construct in health psychology research and practice. Health Psychology Review, 4(2), 57-65.

Hagger, M. S., Wood, C. W., Stiff, C., \& Chatzisarantis, N. (2010). Self-regulation and self-control in exercise: the strength-energy model. International Review of Sport and Exercise Psychology, 3(1), 62-86.

Hair, J. F., Anderson, R. E., Tatham, R. L., \& Black, W. C. (1998). Multivariate data analysis (5th ed.). New Jersey: Prentice-Hall. 
Hampson, S., Glasgow, R., Toobert, D. (1990). Peresonal models of diabetes and their relations to self-care activities. Health Psychology, 9(5), 632-646.

Harrison, T. A., Hindorff, L., \& Kim, H. (2003). Family history of diabetes as a potential public health tool. American Journal of Preventative Medicine, 24, 152-159.

Heijmans, M., \& Ridder, D. d. (1998). Assessing Illness Representations of Chronic Illness: Explorations of their disease-specific nature. Journal of Behavioral Medicine 21(5), 485-503.

Heisler, M., Bouknight, R., Hayward, R., \& Smith, D. M. (2002). The relative importance of physician communication, participatory decision making, and patient understanding in Diabetes self-management. Journal of General Internal Medicine, 17, 243-252.

Howell, D.C. (2010). Statistical methods for psychology ( $7^{\text {th }}$ ed.). Belmont, CA: Thompson Wadsworth.

IBM Coporation (released 2012). IBM SPSS Statistics for Windows Version 21.0. Armonk, NY: IBM Corp.

International Diabetes Federation (2009). Guidelines for Self-monitoring of blood glucose in non-insulin treated type 2 diabetes. Retreived from: http://www.idf.org/webdata/docs/SMBG_EN2.pdf on September 11, 2012.

Jayne, R., \& Rankin, S. (2001). Application of Leventhal's self-regulation model to chinese immigrants with type 2 diabetes. Journal of Nursing Scholarship, 33(1), 53-59. 
Jenkins, N., Hallowell, N., Farmer, A. J., Holman, R. R., \& Lawton, J. (2010). Initiating insulin as part of the treating to target in type 2 diabetes (4-T) trial. Diabetes Care, $33(10), 2178-2180$.

Khunti, K., Skinner, T.C., Heller, S., Carey, M.E., Dallosso, H.M., et al. (2008). Educations and psychological care: Biomedical, lifestyle and psychosocial characteristics of people newly diagnosed with Type 2 diabetes: baseline data from the DESMOND randomized controlled trial. Diabetic Medicine, 25: 14541461.

Kolb, H., Kempf, K., Martin, S., Stumvoll, M., Landgraf, R. (2010). On what evidencebase do we recommend self-monitoring of blood glucose. Diabetes research and clinical practice, 87: 150-156.

Lalic, N., Tankova, T., Nourredine, M., et al. (2012). Value and utility of structured selfmonitoring of blood glucose in real world clinical practice: findings from a multinational observational study. Diabetes technology \& Therapeutics. 14 (4), 338-344.

Lee, Y.-Y., \& Lin, J. L. (2009). The effects of trust in physician on self-efficacy, adherence and diabetes outcomes. Social Science and Medicine, 68, 1060-1068.

Leventhal, H. (1970). Findings and Theory in the study of fear communications. Advances in Experimental Social Psychology, 5(119-186).

Leventhal, H., Leventhal, E. A., \& Contrada, R. J. (1998). Self-regulation, health and behavior: A perceptual-cognitive approach. Psychology \& Health, 13(717-733).

Lu, J, Bu, R.F., Sun, Z.L., Lu, Q.S., Jin, H., et al. (2011). Comparable efficacy of selfmonitoring of quantitative urine glucose with self-monitoring of blood glucose on 
glycaemic control in non-insulin-treated type 2 diabetes. Diabetes Research and Clinical Practice, 93, 179-186.

MacKinnon, D. (2011). Integrating mediators and moderators in research design. Research on Social Work Practice, 21, 675-81.

MacKinnon, D., Lockwood, C., Hoffman, J., et al. (2002). A comparison of methods to test mediation and other interventing variable effects. Psychological Methods. $7(1), 83-104$.

McAndrew, L., Horowitz, C., Lancaster, K., Leventhal, H. (2010) Factors related to perceived diabetes contorl are not related to actual glucose control for minority patients with diabetes.

McAndrew, L., Schneider, S. H., Burns, E., \& Leventhal, H. (2007). Does patient blood glucose monitoring improve diabetes control?: A systematic review of the literature. The Diabetes Educator, 33(6).

Mc Sharry, J., Moss-Morris, R., Kendrick, T. (2011) Illness perceptions and glycaemic control in diabetes: a systematic review with meta-analysis. Diabetic Medicine 28 (11): $1300-1310$.

Moss-Morris, R., Weinman, J., Petrie, K., Horne, R., Cameron, L., Buick, D. (2002) The Revised Illness Perceptions Questionnaire (IPQ-R). Psychology and Health, 17(1) $1-16$.

Murata, G. H., Duckworth, W., Shah, J. H., Wendel, C., \& Hoffman, R. M. (2004). Factors affecting hypoglycemia awareness in insulin-treated type 2 diabetes: The Diabetes Outcomes in Veterans Study (DOVES). Diabetes Research and Clinical Practice, 65, 61-67. 
Nouwen, A., Ford, T., Balan, A. T., Twisk, J., Ruggiero, L., \& White, D. (2011).

Longitudinal motivational predictors of dietary self-care and diabetes control in adults with newly diagnosed type 2 diabetes mellitus. Health Psychology, 30(6), 771-779.

Painter, J., Borba, C., Hynes, M., Mays, D., \& Glantz, K. (2008). The use of theory in health behavior research from 2000 to 2005: a systematic review. Annals of Behavioral Medicine, 35, 358-362.

Parkin, C., Buskirk, A., Hinnen, D., Axel-Schweitzer, M. (2012). Results that matter: Structured vs. unstructured self-monitoring of blood glucose in type 2 diabetes. Diabetes Research and Clinical Practice, 97, 6-15.

Petrie, K. J., \& Weinman, J. A. (1997). Perceptions of health \& illness: current research and applications. the netherlands: harwood academic publishers.

Polonsky, W., Anderson, B., Lohrer, P. , et al. (1995) Assessment of diabetes-related distress. Diabetes Care, 18 (6), 754-760.

Primozic, S., Tavcar, R., Avbelj, M., Dernovsek, M.Z., Oblak, M.R. (2012). Specific cognitive abilities are associated with diabetes self-management behavior among patients with type 2 diabetes. Diabetes Research and Clinical Practice, 95, 48-54.

Raffle, H., Ware, L., Ruhil, A., Hamel-Lambert, J., Denham, S. (2012). Predictors of daily blood glucose monitoring in Appalachian Ohio. American Jounral of Health Behavior, 36(2): 193-202.

Renn, B., Feliciano, L, Segal, D. (2011). The bidirectional relationship of depression and diabetes: a systematic review. Clinical Psychology Review, 31 (8), 1239-1246. 
Robertson, M. (1992). The meaning of compliance: patient perspectives. Qualitative Health Research, 2(1), 7-26.

Robertson, S.M., Amspoker, A.B., Cully, J.A., Ross, E.L., Nail, A.D. (2013). Affective symptoms and change in diabetes self-efficacy and glycaemic control. Diabetic Medicine, 30, e189-e196.

Rodbard, H., Schnell, O., Unger, J., Rees, C., Amstutz, L., et al. (2012). Use of an automated decision support tool optimizes clinicans' ability to interpret and appropriately respond to structured self-monitoring of blood glucose data. Diabetes Care, 35, 693.

Rosenstock, I. M. (1974). The Health Belief Model and preventative helath behavior. In M. H. Becker (Ed.), The health belief model and personal health behavior (pp. 27-59). New Jersey: Slack, Inc.

Rungruangsiripan, M., Sitthimongkol, Y., Maneesriwongul, W., Talley, S., \& Vorapongsathorn, T. (2011). Mediating role of illness representation among social support, therapeutic alliance, experience of medication side effects, and medication adherence in persons with schizophrenia. Acrchives of Psychiatric Nursing, 25(4), 269-283.

Salovey, P., Rothman, A. J., Detweiler, J., \& Wayne, S. (2000). Emotional states and physical health. American Psychologist, 55(1), 110-121.

Scavini, M., Bosi, E., Ceriello, A., Giorgino, F., et al. (2011). Prospective, randomized trial on intensive SMBG management added value in non-insulin treated T2DM patients (PRISMA): a study to determine the effect of a structured SMBG intervention. Acta Diabetol. Online open access: springerlink.com. 
Searle, A., Norman, P., Thompson, R., \& Vedhara, K. (2007). Illness representations among patients with type 2 diabetes and their partners: Relationshpis with selfmanagement behaviors. Journal of Psychosomatic Research, 63, 175-184.

Skinner, T.C., Carey, M.E., Cradock, S., Dallosso, H.M., et al. (2011). Comparison of illness representations dimensions and illness representation clusters in predicting outcomes in the first year following diagnosis of type 2 diabetes: Results from the DESMOND trial. Psychology and Health, 26 (3), 321-335.

Snoek, F. J., Pouwer, F., Welsch, G. W., \& Polonsky, W. H. (2000). Epidemiology/heatlh services/psychosocial research -- Diabetes-related emotional distress in Dutch and U.S. diabetic patients: Cross-cultural validity of the Problem Areas in Diabetes Scale. Diabetes Care, 23(9).

Stephens, J., Carman, J., Brooks, C., Lyons, R., Ford, D., Price, D., Bain, S. (2011). Comment on: Polonsky et al. Structured self-monitoring of blood glucose significantly reduces A1C levels in poorly controlled, noninsulin-treated type 2 diabetes: Results from the Structured Testing Program study. Diabetes Care, 34: e57.

Stetson, B., Schlundt, D., Rothschild, C., Floyd, J., Rogers, W. (2011). Development and validation of the Personal Diabetes Quetionnaire (PDQ): A measure of diabetes self-care behaviors, perceptions and barriers. Diabetes Research and Clinical Practice, 91(3) 321-332.

Taylor, A.B., MacKinnon, D.P., Tein, J. (2008). Tests of the three-path mediated effect. Organizational Research Methods, 11(2), 241-269. 
Tettersell, M. (1993). Asthma patients; knowledge in relation to compliance with drug therapy. Journal of Advanced Nursing, 18(1), 103-113.

Thoolen, B., Ridder, D. d., Bensing, J., Gorter, K., \& Rutten, G. (2008). No worries, no impact? A systematic review of emotional, cognitive, and behavioural responses to the diagnosis of type 2 diabetes. Health Psychology Review, 2(1), 65-93.

Toobert, D., Hampson, S. \& Glascow, R. (2000). The summary of diabetes self-care activities measure: results from 7 studies and a revised scale. Diabetes Care, 23(7), 943-950.

TRIAD Study Group (2002). The translating research into action for Diabetes (TRIAD) study: a multicenter study of diabetes in managed care. Diabetes Care, 25(2), 386-389.

United Kingdom Prospective Diabetes Group. (1991). UK Prospective Diabetes Study (UKPDS): VIII. Study design, progress and performance. Diabetologia, 34, 877890.

United Kingdom Prospective Diabetes Group. (1998). Intensive blood-glucose control with sulphonylureas or insulin compared with conventional treatment and risk of complications in patients with type 2 diabetes (UKPDS 33).

United States Census Data (2010). United States Census, 2010: Floyd County, Indiana. Retrieved from: http://www.stats.indiana.edu/topic/census.asp on September 12, 2012.

Walter, F. M., \& Emery, J. (2006). Perceptions of family history across common diseases: a qualitative study in primary care. Family Practice, 23, 472-480. 
Wang, J., Zgibor, J., Matthews, J., Prochownik, D.C., et al. (2007). Self-monitoring of blood glucose is associated with problem-solving skills in hyperglycemia and hypoglycemia. The Diabetes Educator, 38: 201-214.

Ware, J. E., Kosinski, M., \& Keller, S. D. (March 01, 1996). A 12-Item Short-Form Health Survey: Construction of Scales and Preliminary Tests of Reliability and Validity. Medical Care, 34, 3, 220-233.

Welch, G., Weinger, K., Anderson, B., Polonsky W. (2003) Responsiveness of the Problem Areas in Diabetes (PAID) questionnaire. Diabetic Medicine, 20 (1): 6972.

Welch, G. W., Jacobson, A. M., \& Polonsky, W. H. (1997). The Problem Areas in Diabetes Scale. And evaluation of its clinical utility. Diabetes Care, 20(5), 760766.

Wing, R., Epstein, L.H., Nowalk, M.P., Lamparski, D. M. (1986). Behavioral SelfRegulation in the Treatment of Patients with Diabetes Mellitus. Psychological Bulletin. 99 (1), 78-89.

Zulman, D., Rosland, A., Choi, H., Langa, K., Heisler, M. (2012). The influence of diabetes psychosocial attributes and self-management practices on change in diabetes status. Patient Education and Counseling, 87, 74-80. 


\section{Appendix 1 Guide to Abbreviations}

A1c: $\quad$ Abbreviation of Hemoglobin A1c, a physiological marker of long-term blood glucose control, currently the standard marker in Diabetes diagnosis and disease progression

ADA: $\quad$ American Diabetes Association

CSM: $\quad$ Common Sense Model (illness representation component of the SelfRegulation Model)

HCP: $\quad$ Healthcare provider

IDF: $\quad$ International Diabetes Federation

JDC: $\quad$ Joslin Diabetes Center

SMBG: $\quad$ Self-monitoring of blood glucose

SRM: $\quad$ Self-regulation model (of illness behavior)

T1DM: $\quad$ Type 1 Diabetes Mellitus, previously referred to as insulin dependent Diabetes Mellitus (IDDM)

T2DM: $\quad$ Type 2 Diabetes Mellitus, previously referred to non-insulin dependent Diabetes Mellitus (NIDDM) 
Appendix 2

Questionnaire Packet

\section{GENERAL BACKGROUND INFORMATION}

1. Today's date: (month/day/year)

2. How old are you? (years old)

3. Gender
Female
Male

4. How tall are you?

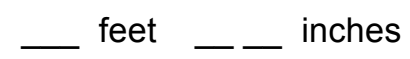

5. How much do you currently weigh?

_

6. Ethnic group (check one box):

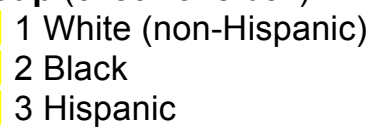

4 Asian

5 Other Specify Below

7. Marital status (check one box):
1 Never married
2 Currently married
3 Separated

5 Widowed

4 Divorced

8. Current living arrangement (check one box):

1 Live alone

2 Live with spouse/partner

3 Live with spouse/partner and children

4 Live with children (no spouse/partner)

9. Level of school completed (check one box):

1 Less than 7 th grade

5 Partial college

2 Junior High School (7th, 8th, \& 9th grade)

3 Partial high school (10th or 11th grade)

5 Live with roommate who is not partner

6 Live with parents

7 Other or specialized training 6 College or university graduate 7 Graduate professional training

4 High School graduate (Includes G.E.D.) (graduate degree) 
10. Approximate annual gross income for your household: (check one number) (Remember all information you provide will remain completely confidential)

$$
\begin{array}{ll|ll}
1 & \text { Less than } \$ 10,000 & 4 & \$ 40,000-\$ 59,999 \\
2 & \$ 10,000-\$ 19,999 & 5 & \$ 60,000-\$ 100,000 \\
3 & \$ 20,000-\$ 39,999 & 6 & \text { Greater than } \$ 100,000
\end{array}
$$

11. Which category best describes your usual occupation? If you are not currently employed, which category best describes your LAST job? (check one number)

1 Professional (e.g., teachers/professors, nurses, lawyers, physicians, \& engineers)

2 Manager/Administrator (e.g., sales managers)

3 Clerical (e.g., secretaries, clerks or mail carriers)

4 Sales (e.g., sales persons, agents \& brokers)

5 Service (e.g., police, cooks, waitress, or hairdressers)

6 Skilled Crafts, Repairer (e.g., carpenters)

7 Equipment or Vehicle Operator (e.g., truck drivers)

8 Laborer (e.g., maintenance factory workers)

9 Farmer (e.g., owners, managers, operators or tenants)

10 Member of the military

11 Homemaker (with no job outside the home)

12 Other (please describe)

12. Current employment situation (check all that apply):

1 Full time at job

2 Part time at job

3 On leave with pay

4 On leave without pay

5 Disabled

6 Seeking work

7 Retired

8 Homemaker

9 Student 


\section{YOUR VIEWS ABOUT YOUR DIABETES (IPQ-R)}

Listed below are a number of symptoms that you may or may not have experienced since your diabetes. Please indicate by circling Yes or No, whether you have experienced any of these symptoms since your diabetes, and whether you believe that these symptoms are related to your diabetes.

\begin{tabular}{|c|c|c|c|c|}
\hline \multirow[b]{2}{*}{ Pain } & \multicolumn{2}{|c|}{$\begin{array}{l}\text { I have experienced } \\
\text { this symptom } \\
\text { since my diabetes }\end{array}$} & \multicolumn{2}{|c|}{$\begin{array}{l}\text { This symptom is } \\
\text { related to } m y \\
\text { diabetes }\end{array}$} \\
\hline & Yes & No & Yes & No \\
\hline Sore Throat & Yes & No & Yes & No \\
\hline Nausea & Yes & No & Yes & No \\
\hline Breathlessness & Yes & No & Yes & No \\
\hline Weight Loss & Yes & No & Yes & No \\
\hline Fatigue & Yes & No & Yes & No \\
\hline Stiff Joints & Yes & No & Yes & No \\
\hline Sore Eyes & Yes & No & Yes & No \\
\hline Wheeziness & Yes & No & Yes & No \\
\hline Headaches & Yes & No & Yes & No \\
\hline Upset Stomach & Yes & No & Yes & No \\
\hline Sleep Difficulties & Yes & No & Yes & No \\
\hline Dizziness & Yes & No & Yes & No \\
\hline Loss of Strength & Yes & No & Yes & No \\
\hline
\end{tabular}


We are interested in your own personal views of how you now see your current diabetes.

Please indicate how much you agree or disagree with the following statements about our diabetes by checking the appropriate box.

\begin{tabular}{|c|c|c|c|c|c|c|}
\hline & Views about your Diabetes & $\begin{array}{l}\text { Strongly } \\
\text { disagree }\end{array}$ & Disagree & $\begin{array}{c}\text { Neither } \\
\text { agree nor } \\
\text { disagree }\end{array}$ & Agree & $\begin{array}{c}\text { Strongly } \\
\text { Agree }\end{array}$ \\
\hline 1 & This diabetes will pass quickly & & & & & \\
\hline 2 & $\begin{array}{l}\text { I expect to have this diabetes for the } \\
\text { rest of my life }\end{array}$ & & & & & \\
\hline 3 & My diabetes is a serious condition & & & & & \\
\hline 4 & $\begin{array}{l}\text { My diabetes has major consequences } \\
\text { on my life }\end{array}$ & & & & & \\
\hline 5 & $\begin{array}{l}\text { My diabetes does not have much } \\
\text { effect on my life }\end{array}$ & & & & & \\
\hline 6 & $\begin{array}{l}\text { My diabetes strongly affect the way } \\
\text { others see me }\end{array}$ & & & & & \\
\hline 7 & $\begin{array}{l}\text { Me diabetes has serious financial } \\
\text { consequences }\end{array}$ & & & & & \\
\hline 8 & $\begin{array}{l}\text { My diabetes causes difficulties for } \\
\text { those who are close to me }\end{array}$ & & & & & \\
\hline 9 & $\begin{array}{l}\text { There is a lot which I can do to control } \\
\text { my symptoms }\end{array}$ & & & & & \\
\hline 10 & $\begin{array}{l}\text { What I do can determine whether my } \\
\text { diabetes gets worse }\end{array}$ & & & & & \\
\hline 11 & $\begin{array}{l}\text { The course of my diabetes depends } \\
\text { on me }\end{array}$ & & & & & \\
\hline 12 & Nothing I do will affect my diabetes & & & & & \\
\hline 13 & $\begin{array}{l}\text { I have the power to influence my } \\
\text { diabetes }\end{array}$ & & & & & \\
\hline 14 & $\begin{array}{l}\text { My actions will have no affect on the } \\
\text { outcome of my diabetes }\end{array}$ & & & & & \\
\hline 15 & My diabetes will improve in time & & & & & \\
\hline 16 & $\begin{array}{l}\text { There is very little that can be done to } \\
\text { improve my diabetes }\end{array}$ & & & & & \\
\hline 17 & $\begin{array}{l}\text { My treatment will be effective in } \\
\text { curing my diabetes }\end{array}$ & & & & & \\
\hline
\end{tabular}




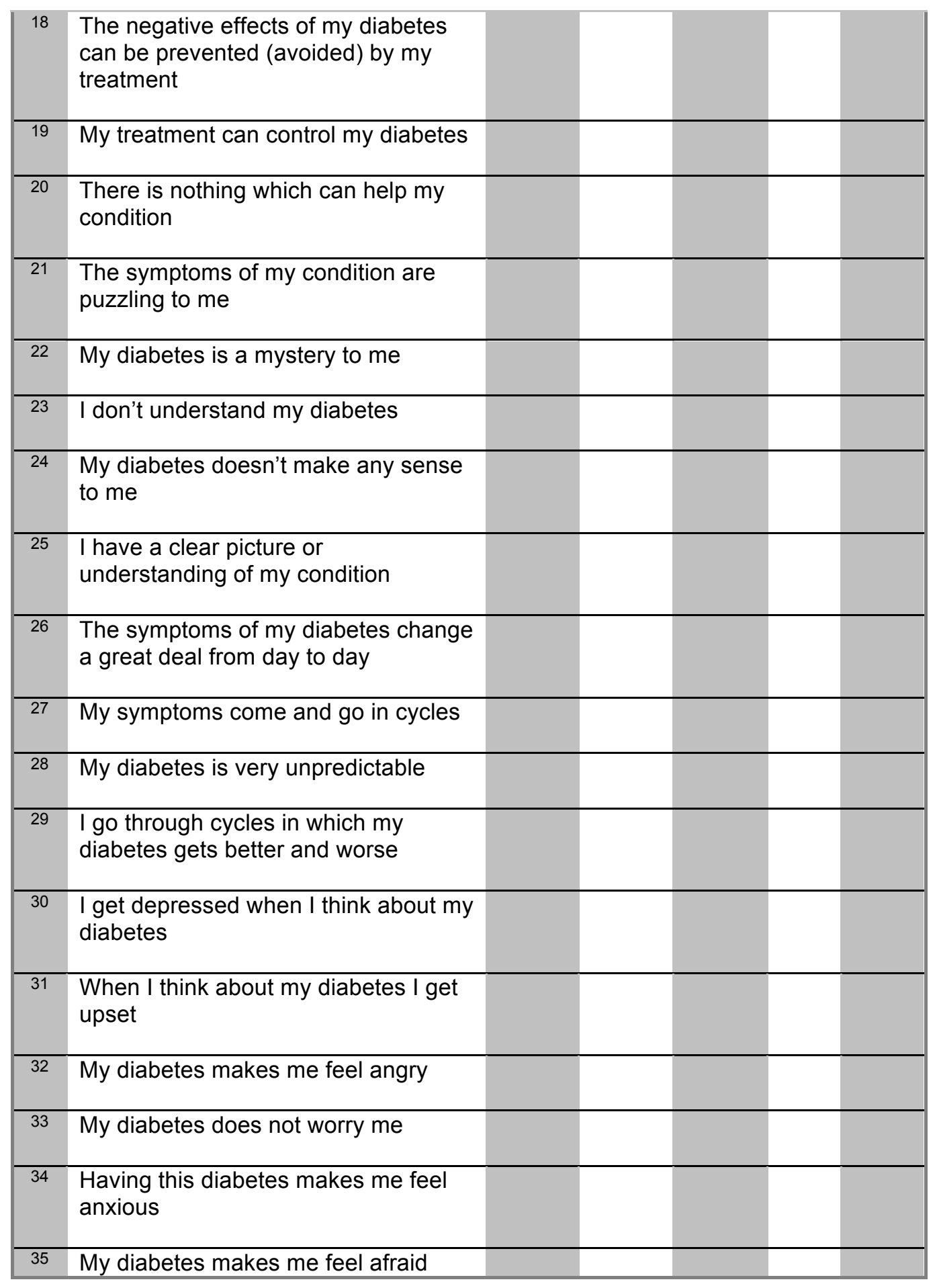

Please continue to the next page $\rightarrow$ 
1. To what extent are you using your blood sugar testing to decide what to do, like what to eat or how to be physically active, to manage your diabetes?

0

Not at all
1

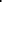

2

3
4

extremely

\section{SDSCA}

The two questions below ask you about your diabetes self-care during the last 7 days. If you were sick during the past 7 days, please think back to the last 7 days that you were not sick.

2. On how many days of the last SEVEN DAYS did you test your blood sugar?

$\begin{array}{llllllll}0 & 1 & 2 & 3 & 4 & 5 & 6 & 7\end{array}$

3. On how many of the last SEVEN DAYS did you test your blood sugar the number of times recommended by your health care provider?

$\begin{array}{llllllll}0 & 1 & 2 & 3 & 4 & 5 & 6 & 7\end{array}$

\section{Problem Areas In Diabetes (PAID) Questionnaire}

INSTRUCTIONS: Which of the following diabetes issues are currently a problem for you? Circle the number that gives the best answer for you.

Please provide an answer for each question.

\begin{tabular}{|l|l||l|l||l||}
\hline & $\begin{array}{l}\text { Not a } \\
\text { Problem }\end{array}$ & $\begin{array}{l}\text { Rarely a } \\
\text { Problem }\end{array}$ & $\begin{array}{l}\text { Often a } \\
\text { Problem }\end{array}$ & $\begin{array}{l}\text { Always a } \\
\text { Problem }_{4}\end{array}$ \\
\hline $\begin{array}{l}\text { 1. Worrying about the future and the } \\
\text { possibility of serious complications. }\end{array}$ & & & \\
\hline $\begin{array}{l}\text { 2. Feeling guilty or anxious when you } \\
\text { get off track with your diabetes } \\
\text { management. }\end{array}$ & & & \\
\hline $\begin{array}{l}\text { 3. Feeling scared when you think } \\
\text { about living with diabetes. }\end{array}$ & & & \\
\hline $\begin{array}{l}\text { 4. Feeling discouraged about your } \\
\text { diabetes regimen. }\end{array}$ & & & \\
\hline $\begin{array}{l}\text { 5. Worrying about low blood sugar } \\
\text { reactions. }\end{array}$ & & & \\
\hline $\begin{array}{l}\text { 6. Feeling constantly burned-out by } \\
\text { the constant effort to manage } \\
\text { diabetes. }\end{array}$ & & & \\
\hline
\end{tabular}




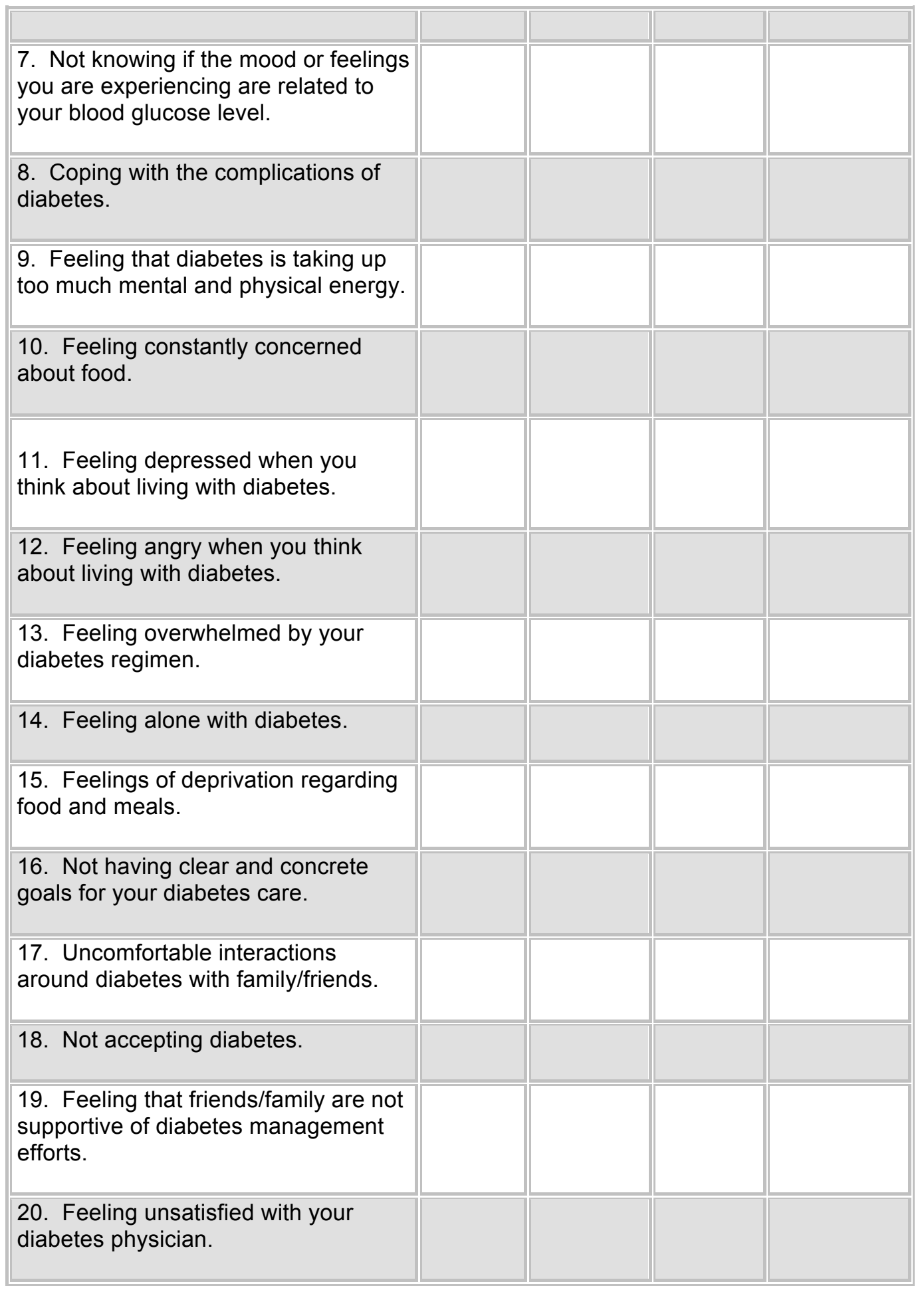




\section{CURRICULUM VITA}

NAME: Jennifer Elizabeth Floyd Ward

ADDRESS: Department of Psychological \& Brain Sciences

317 Life Sciences

University of Louisville

Louisville, KY 40292

EDUCATION

\& TRAINING: $\quad$ Ph.D., Clinical Psychology

University of Louisville, Louisville, KY

$2009-2014$

M.A., Clinical Psychology

University of Louisville, Louisville, KY

$2009-2012$

B.S., Psychology, Studio Art

Centre College, Danville, KY

$2002-2006$

\section{PROFESSIONAL SOCIETIES:}

2009-Present Kentucky Psychological Association

2010-Present Society of Behavioral Medicine, Special interest groups: Health Decision Making, Student, Diabetes, Physical Activity, Ethnic Minority and Multicultural Health, Evidence-Based Behavioral Med

2013-Present Association of Psychologists in Academic Health Settings

2013-Present American Psychological Association, Division 38: Health Psychology

\section{PUBLICATIONS:}

2014 Ward, J., Stetson, B., Mokshagundam, S. (2013) Patient Perspectives on Self-monitoring of Blood Glucose: Perceived Recommendations, Behaviors and Barriers in a clinic sample of adults with Type 2 Diabetes (Under revision for Journal of Diabetes and Metabolic Disorders). 
2011 Stetson, B., Schlundt, D., Rothschild, C., Floyd, J., Rogers, W., Mokshagundam, S. (2011) Development and validation of the Personal Diabetes Questionnaire (PDQ): A measure of diabetes self-care behaviors, perceptions and barriers. Diabetes Research and Clinical Practice. vol 91, pp 321-332.

Under Stetson, B.A, Ritchie, C.S., Adams, K., Marshall, K., Bonner, J.E., Ward,

Revision J.E. (Under Revision). Associations of functional status, pain, and mood with dependence in Activities of Daily Living in urban dwelling older women.

Under Rogers, W., Stetson, B., Bonner, J., Rothschild, C., Meyer, J., Krishnasamy, Revision S., Richardson, K., Ward, J.E., Mokshagundam, S.P. (Under Revision). Social isolation and neighborhood characteristics in low-income adults with type 2 diabetes: Relation to health indicators and quality of life.

NATIONAL MEETING PRESENTATIONS:

2014 Ward, J.E., McDonough, S.R., Stetson, B.A., McKiel, C., DeVore, R., Gonzalez, C., Mokshagundam, S.P. (2014). Illness coherence supports decision-making and better coping in insulin treated type 2 diabetes. Submitted for presentation at the 36th annual meeting of the Society of Behavioral Medicine, San Antonio, TX, April 2015.

2014 Knight, K.M., McDonough, S.R., Ward, J.E., Stetson, B.A., Mokshagundam, S.P. (2014). Mediation effects of health-related coping and blood glucose control in adults with type 1 diabetes. Submitted for presentation at the 36th annual meeting of the Society of Behavioral Medicine, San Antonio, TX, April 2015.

2014 Ward, J., McDonough, S.R., DeVore, R., Stethan, A., Mokshagundam, S.P., Stetson, B.A. (2014). Self-monitoring of blood glucose use as decision-making tool mediates relationship between adherence and glucose control in insulintreated Type 2 diabetes. Presented at the $35^{\text {th }}$ annual meeting of the Society of Behavioral Medicine, Philadelphia, PA.

2014 Stetson, B., Schlundt, D., Ward, J., Knight, H., Cooper, J., Krishnasamy, S., Mokshagundam, S. (2014). Reliability and validity of a brief diabetes self-care measure with low-income, underserved adults. Presented at the 35th annual meeting of the Society of Behavioral Medicine, Philadelphia, PA.

2014 Knight, H., McDonough, S., Ward, J., Cooper, J., Stetson, B., \& Mokshagundam, S. (2014). Mediational effects of stress and social support on diet in women with diabetes and hyperlipidemia. 35th annual meeting of the Society of Behavioral Medicine, Philadelphia, P 
2014 Cooper, J., Stetson, B., Knight, H., McDonough, S., Ward, J. \& Mokshagundam, S. (2014). Self-reported Health Behaviors and Barriers Discriminate Between Targeted Levels of Glycemic Control in a Diabetes Clinical Sample. Presented at the 35th annual meeting of the Society of Behavioral Medicine, Philadelphia, PA.

2013 Ward, J.E., Stetson, B., Krishnasamy, S., Mokshagundam, S.P. (April, 2012). Having a target for glucose control is associated with better diabetes-specific and general coping in underserved adults with type 2 diabetes. Presented at the $34^{\text {th }}$ Annual Meeting of the Society of Behavioral Medicine, San Francisco, CA.

2013 Cooper, J., Stetson, B., Ward, J.E., Knight, H., Krishnasamy, S., Mokshagundam, S.P. (April, 2012). Levels of Provider Care and Health Behaviors in Underserved Adults with Type 2 Diabetes. Presented at the $34^{\text {th }}$ Annual Meeting of the Society of Behavioral Medicine, San Francisco, CA.

2013 Knight, H.M.,Stetson, B., Cooper, J., Ward, J., Krishnasamy, S., Mokshagundam, S.P. (April, 2012). Diet Adherence and Readiness to Change in Underserved Adults with Type 2 Diabetes. Presentated at the $34^{\text {th }}$ Annual Meeting of the Society of Behavioral Medicine, San Francisco, CA.

2012 Floyd, J., Stetson, B., Krishnasamy, S., Mokshagundam, S. (2012). Self-care and Quality of Life in Low-Income, Medically Underserved Adults with Type 2 Diabetes. Annals of Behavioral Medicine, 43, Suppl 1, s100.

2012 Spille, S., Stetson, B., Floyd, J., Mokshagundam,S.P. (April 2012) Smoking Status and Distress, Self-care and indices of Diabetes Control. Rapid Communication Presentation at the $33^{\text {rd }}$ Annual Meeting of the Society of Behavioral Medicine, New Orleans, LA.

2011 Floyd, J., Stetson, B., Rogers, W., Mokshagundam, S. (April 2011) Gender and Age Differences in Barriers to Monitoring Blood Glucose in Type 2 Diabetes. Society of Behavioral Medicine, Washington DC, April, 2011.

2011 Spille, S., Stetson, B., Floyd, J.B., Mokshagundam, S. (April 2011). Smoking Status and Distress, Self-care and indices of Diabetes Control. Society of Behavioral Medicine, Washington DC, April, 2011.

2010 Stetson, B., Edgmon, B., Floyd, J., Rogers, W., Spille, S., Mokshagundam, S. Follow-up of Depressive Symptomology in a Clinic Sample of Adults with Diabetes

2010 Stetson, B., Schlundt, D., Rothschild, C., Rogers, W., Floyd, J.B., Mokshagundam, S.P., Krishnasamy, S. (2010). Reliability and Validity of a Theoretically-Based Diabetes Self-Management Assessment Tool. Society of Behavioral Medicine, Seattle,WA. 
2006 Floyd, J.B. Smoking and Depression in British vs. American University Students. Tri-State Undergraduate Psychology Research Conference. West Virginia. 